\title{
9 Structural basis for Cas9 off-target activity
}

13 Martin Pacesa ${ }^{1}$, Chun-Han Linn ${ }^{2,3}$, Antoine Cléry ${ }^{4}$, Katja Bargsten ${ }^{1,5}$, Matthew J.

14 Irby $^{2}$, Frédéric H.T. Allain ${ }^{4}$, Peter Cameron ${ }^{2,6}$, Paul D. Donohoue ${ }^{2}$, Martin Jinek ${ }^{1}$

Address:

${ }^{1}$ Department of Biochemistry, University of Zurich, Winterthurerstrasse 190, CH-8057 Zurich,

18 Switzerland

$19{ }^{2}$ Caribou Biosciences, 2929 Seventh Street \#105, Berkeley, CA 94710, United States

$20{ }^{3}$ Present address: LinkedIn, Sunnyvale, CA 94085, United States

$21{ }^{4}$ Institute of Biochemistry, ETH Zurich, Hönggerbergring 64, CH-8093 Zurich, Switzerland

$22 \quad{ }^{5}$ Present address: leadXpro AG, Villigen, Switzerland

23 'Present address: Spotlight Therapeutics, Hayward, CA 94545, United States 


\section{Abstract}

The target DNA specificity of the CRISPR-associated genome editor nuclease Cas9 is determined by complementarity to a 20-nucleotide segment in its guide RNA. However, Cas9 can bind and cleave partially complementary off-target sequences, which raises safety concerns for its use in clinical applications. Here we report crystallographic structures of Cas9 bound to bona fide off-target substrates, revealing that off-target binding is enabled by a range of non-

34 canonical base pairing interactions and preservation of base stacking within the guide-off-target heteroduplex. Off-target sites containing single-nucleotide deletions relative to the guide RNA are accommodated by base skipping rather than RNA bulge formation. Additionally, PAMdistal mismatches result in duplex unpairing and induce a conformational change of the Cas9

38 REC lobe that perturbs its conformational activation. Together, these insights provide a 39 structural rationale for the off-target activity of Cas9 and contribute to the improved rational 40 design of guide RNAs and off-target prediction algorithms. 


\section{Introduction}

Cas9, the effector nuclease of prokaryotic Type II CRISPR adaptive immune systems (Makarova et al., 2020), cleaves double-stranded DNA substrates complementary to a guide CRISPR RNA (crRNA) (Jinek et al., 2012). By changing the sequence of the guide RNA, the target DNA specificity of the CRISPR-Cas9 system is readily programmable (Jinek et al., 2012), a feature that has been widely exploited for genome engineering applications (Anzalone et al., 2020). Cas9 functions in conjunction with a trans-activating crRNA (tracrRNA), which is required both for crRNA loading and subsequent DNA binding and cleavage (Deltcheva et al., 2011; Jinek et al., 2012). Target DNA binding and cleavage is further dependent on the presence of a protospacer-adjacent motif (PAM) flanking the target sequence (Anders et al., 2014; Jinek et al., 2012). Due to its high activity and 5'-NGG-3' PAM specificity, Streptococcus pyogenes Cas9 (SpCas9) remains the most widely used CRISPR-Cas nuclease for gene editing applications. However, despite a high intrinsic accuracy in generating targeted DNA breaks, SpCas9 can nevertheless cleave genomic DNA sequences with imperfect complementarity to the guide RNA, resulting in off-target editing (Cameron et al., 2017; Hsu et al., 2013; Pattanayak et al., 2013; Tsai et al., 2015). The off-target activity of SpCas9, as well as other Cas9 enzymes, thus presents a safety concern for their therapeutic applications.

Off-target sites typically contain one or several nucleobase mismatches relative to the guide RNA (Cameron et al., 2017; Tsai et al., 2017; Tsai et al., 2015). Recent studies have established that the type of mismatch, its positioning within the heteroduplex, and the total number of mismatches are important determinants of off-target DNA binding and cleavage (Boyle et al., 2017; Boyle et al., 2021; Doench et al., 2016; Jones et al., 2020; Zhang et al., 2020). PAM-proximal mismatches within the seed region of the guide RNA-target DNA strand heteroduplex typically have a dramatic impact on substrate DNA binding and R-loop formation (Boyle et al., 2021; Ivanov et al., 2020; Singh et al., 2016; Zhang et al., 2020). In contrast, 
PAM-distal mismatches are compatible with stable binding; however, their presence often results in the formation of a catalytically incompetent complex (Boyle et al., 2021; Dagdas et al., 2017; Ivanov et al., 2020; Jones et al., 2020; Sternberg et al., 2015; Yang et al., 2018; Zhang et al., 2020). In addition, Cas9 has been shown to cleave off-target substrates containing insertions or deletions relative to the guide RNA sequence, which have been proposed to be recognized through the formation of nucleotide "bulges" in the guide RNA-target DNA heteroduplex (Boyle et al., 2021; Cameron et al., 2017; Doench et al., 2016; Jones et al., 2020; Lin et al., 2014; Tsai et al., 2015).

Numerous computational tools have been developed to predict possible genomic offtarget sites based on sequence similarity (Bae et al., 2014; Stemmer et al., 2015). However, the majority of actual off-target cleavage events remain unpredicted (Cameron et al., 2017; Tsai et al., 2015). Furthermore, although Cas9 is able to bind genomic sites harbouring as few as five complementary nucleotides, only a relatively small number of off-target sites are actually cleaved and result in detectable off-target editing in cells (Kuscu et al., 2014; O'Geen et al., 2015; Wu et al., 2014). Several structures of target-bound Cas9 complexes have been determined to date (Anders et al., 2014; Jiang et al., 2016; Nishimasu et al., 2014; Zhu et al., 2019) that have shed light on the mechanism of on-target binding and cleavage. However, the same processes for off-target sites remain poorly understood.

To elucidate the mechanism of mismatch tolerance of Cas9, we determined crystal structures of a comprehensive set of bona fide off-target-bound complexes. These structures reveal that the formation of non-canonical base pairs and preservation of heteroduplex shape underpin the off-target tolerance of Cas9. We also observe that consecutive mismatches in the seed region can be accommodated by base skipping of a guide RNA nucleotide, as opposed to nucleotide bulging. Finally, the structure of an off-target complex containing three PAM-distal mismatches exhibits REC2/3 domain rearrangements, which likely perturbs conformational 
92 activation of Cas9 and thus modulates cleavage efficiency. Taken together, our structural data

93 reveal the diversity of mechanisms enabling off-target recognition and lay the foundation for

94 engineering optimized CRISPR-Cas9 complex designs for gene editing. 


\section{Results}

\section{In vitro profiling reveals diversity of Cas9 off-targets}

Multiple studies have investigated the off-target activity of Cas9, suggesting context-dependent tolerance of nucleobase mismatches between the guide RNA and off-target DNA sequences (Boyle et al., 2021; Cameron et al., 2017; Lazzarotto et al., 2020; Tsai et al., 2015; Zhang et al., 2020). To investigate the effect of mismatches on Cas9 binding and cleavage, we performed the SITE-Seq ${ }^{\circledR}$ assay (Cameron et al., 2017) to define the off-target landscapes of 12 wellstudied guide RNAs to select suitable off-targets for further evaluation (Table S1, Table S2) (Figure S1A-B). The SITE-Seq assay analysis revealed a total of 3,848 detectable off-target sites at the highest Cas9 ribonucleoprotein (RNP) concentration, with a total of 21,732 base mismatches and a median of 5 mismatches per off-target site (Figure S1C). The detected mismatches covered all possible base mismatch combinations and were distributed throughout the length of the guide RNA-target DNA heteroduplex (Figure S1D-E).

To probe the thermodynamics of on- and off-target substrate DNA binding and the kinetics of DNA cleavage, we focused on a subset of four guide RNAs (AAVS1, FANCF, PTPRC-tgt2, and TRAC) and a total of 15 bona fide off-target sites detectable in vivo (Cameron et al., 2017; Donohoue et al., 2021; Tsai et al., 2017; Tsai et al., 2015) (Figure 1A) that covered all 12 possible base mismatch types. Nuclease activity assays using synthetic DNA substrates with fluorophore-labeled target strand (TS) revealed that all selected off-target sequences were cleaved slower than the corresponding on-target substrates, with 20-500-fold reductions in the calculated rate constants (Figure 1B, Figure S2A). To distinguish whether the cleavage defects were due to slower R-loop formation or perturbations in downstream steps, including conformational activation of the nuclease domains, we also quantified cleavage kinetics using PAMmer DNA substrates (Anders et al., 2014; O'Connell et al., 2014) in which the on-/offtarget sequence was single-stranded. These experiments revealed that the slower cleavage 
kinetics of most off-target substrates was due to perturbed R-loop formation (Figure 1B, Figure

121 S2B). However, for some off-targets, notably AAVS1 off-targets \#2 and \#5, FANCF off-targets \#3, \#4, and \#6, and PTPRC-tgt2 off-target \#1, the rate of PAMmer substrate cleavage was more than 100-fold slower as compared to their respective on-target sequences (Figure 1B, Figure

S2B). This indicates that these off-target mismatches may additionally cause perturbations in the conformational activation checkpoint downstream of guide-target hybridization or inhibit cleavage by direct steric hindrance of the Cas9 HNH domain (Chen et al., 2017; Dagdas et al., 2017). (switchSENSE) methodology revealed perturbations in binding affinities for most off-targets as compared to the respective on-target sequences (Figure 1B, Data S1). Notably, the

131 reductions in binding affinities were almost entirely due to increased dissociation rates ( $\mathrm{k}_{\mathrm{off}}$ ),

132 while on-binding rates $\left(\mathrm{k}_{\mathrm{on}}\right)$ were largely unperturbed (Figure 1B), indicating that most of the 133 off-target mismatches in our data set promote DNA dissociation, likely due to R-loop collapse.

134 However, there was little correlation between the observed reductions in cleavage rates and 135 binding constants (Figure S2C), confirming that the molecular basis for off-target 136 discrimination by Cas9 is not based on substrate binding alone, in agreement with prior studies 137 (Boyle et al., 2021; Chen et al., 2017; Dagdas et al., 2017; Jones et al., 2020; Yang et al., 2018; 138 Zhang et al., 2020). The dissociation rate $\left(\mathrm{k}_{\text {off }}\right)$ correlated significantly only with the number of 139 mismatches located in the seed region $\left(\mathrm{R}^{2}=0.46, \mathrm{p}=0.001\right)$ (Figure S2C), suggesting that off140 targets with mismatches in the seed are regulated mainly through R-loop collapse and non141 target strand (NTS) rehybridization (Boyle et al., 2017; Gong et al., 2018; Singh et al., 2016; 142 Sternberg et al., 2014).

143 Finally, we correlated the measured cleavage rate constants $\left(\mathrm{k}_{\mathrm{obs}}\right)$ with predicted data 144 based on a leading biophysical model of Cas9 off-target cleavage that accounts for mismatch 
145 number and position using position-dependent penalties and includes position-independent

146 weights for mismatch type (Jones et al., 2020). Although there was good overall correlation

147 between the model and our data $\left(\mathrm{R}^{2}=0.46, \mathrm{p}=0.004\right)$ (Figure S2C), there were nevertheless

148 several prominent outliers (AAVS1 off-target \#2 and off-target \#3, and FANCF off-target \#4),

149 suggesting that accurate modelling of off-target interactions will require accounting for

150 position-specific effects of individual mismatch types (Figure S2D).

151 Taken together, these results indicate that bona fide off-target substrates exhibit

152 significantly perturbed kinetics of substrate DNA binding and cleavage. Moreover, the

153 magnitude of the perturbation is dependent not only on the number and position of mismatches

154 in the off-target sequence but also on mismatch type, in agreement with recent studies (Boyle

155 et al., 2021; Doench et al., 2016; Jones et al., 2020; Zhang et al., 2020). Moreover, the off-target

156 activity cannot be accurately predicted by biophysical off-target activity models that account

157 for mismatch type in a position-independent manner, implying that mismatches have position-

158 specific and context-dependent effects. This further highlights the need to understand the

159 molecular principles of Cas9 off-target recognition at the structural level.

160 Crystallographic analysis of off-target interactions

161 To obtain insights into the structural basis of off-target recognition and mismatch tolerance, we

162 employed a previously described approach (Anders et al., 2014) to co-crystallize Cas9 with

163 sgRNA guides and partially duplexed off-target DNA substrates (Figure 1C). Focusing on our

164 set of $A A V S 1, F A N C F, P T P R C$-tgt2 and TRAC off-targets (Figure 1A), covering all 12 possible

165 mismatch types, we determined a total of 15 off-target complex structures at resolutions of

$1662.25-3.30 \AA$ (Figure 1C, Table S3). For the $A A V S 1, F A N C F$ and $T R A C$ guide RNAs, we also

167 determined the structures of the corresponding on-target complexes; the structure of the

168 PTPRC-tgt2 on-target complex could not be determined due to insufficient diffraction of the

169 crystals. Overall, the off-target complex structures have very similar conformations, with the 
Cas9 polypeptide backbone superimposing with a mean root mean square deviation of $0.41 \AA$ over $1330 \mathrm{C} \alpha$ atoms (as referenced to the FANCF on-target complex structure, excluding FANCF off-target \#4, as discussed below). Of note, the AAVS1 on-target complex structure reveals substantial repositioning of the REC2 domain, where it undergoes a $12^{\circ}$ rotation (as compared to the FANCF and TRAC on-target complexes) (Figure S3A), with concomitant shortening of the $\alpha$-helix comprising residues 301-305 and restructuring of the loop comprising residues 175-179 (Figure S3B), enabled by the absence of crystal contacts involving the REC2 and REC3 domains.

However, the structures display considerable local variation of the RNA-TS DNA heteroduplex conformation. Base base pairing and base stacking are mostly preserved throughout the guide RNA-TS DNA heteroduplexes (Table S4), with the exception of positions 1-3 within the PAM-distal end of the guide-TS duplex, where the presence of mismatches

182 results in duplex unpairing. This is observed in AAVS1 off-target \#2 and \#4, FANCF off-target

$183 \# 4$ and $\# 5$, and TRAC off-target \#1 complexes (Figure S4). Despite the observed conformational variation, the off-target structures preserve almost all intermolecular contacts between the Cas9 protein and the bound nucleic acids, further underscoring the structural plasticity of Cas9 in accommodating mismatch-induced distortions in the guide RNA-TS DNA 187 heteroduplex.

188 Together, these observations indicate that the crystal form used for determination of the 189 off-target complex structures is sufficiently plastic to accommodate conformational changes 190 resulting from the presence of base mismatches in the guide RNA-TS DNA heteroduplex and 191 imply that the observed structural effects of guide RNA-TS DNA base mismatches provide a 192 true depiction of off-target DNA binding. In addition, the $\mathrm{HNH}$ and REC2/3 domain 193 conformations observed in the crystal structures are similar to those observed in the 16-bp 194 heteroduplex, pre-checkpoint state determined by cryo-EM (Pacesa and Jinek, 2021). 
196 Close inspection of the 15 Cas9 off-target complex structures reveals that a substantial fraction

197 of off-target base mismatches (34 out of 49) is accommodated by non-canonical base pairing

198 interactions that preserve at least one hydrogen bond between the guide and off-target bases.

199 The most common off-target mismatches, both in our data set (Table S1, Table S2) and as 200 reported by other studies (Boyle et al., 2017; Doench et al., 2016; Hsu et al., 2013; Jones et al., 201 2020; Pattanayak et al., 2013; Zhang et al., 2020), are rG-dT (Figure S5) and rU-dG (Figure 202 2A, Figure S6), which have the potential to form wobble base pairs (Kimsey et al., 2015). 203 Indeed, all rG-dT mismatches in the determined structures are accommodated by wobble base 204 pairing. At duplex positions 4 (AAVS1 off-target \#1 and \#5), 13 (FANCF off-target \#2 and \#7) 205 and 15 (TRAC off-target \#1), the dT base undergoes a $\sim 1 \AA$ shear displacement into the major 206 groove of the guide-TS DNA heteroduplex to form the wobble base pair (Figure S5), whereas 207 at duplex position 2 (in TRAC off-target \#1 and \#2), wobble base pairing is enabled by a minor 208 groove displacement of the $\mathrm{rG}$ base. In contrast, $\mathrm{rU}-\mathrm{dG}$ mispairs in the determined structures 209 exhibit considerable structural variation. At duplex position 10 in the FANCF off-target \#2 and 210 \#4 complexes, the $\mathrm{rU}$ base is able to undergo the major groove displacement required for 211 wobble base-pairing (Figure 2A, Figure S6A). In contrast, at duplex position 5 in $F A N C F$ off212 target \#1, \#3 and \#6 complexes, the backbone of the RNA strand makes extensive contacts with 213 Cas9 (Figure S6B-D). As a result, the rU-dG mispairs are instead accommodated by 214 compensatory shifts of the dG base to maintain hydrogen-bonding interactions (Figure S6B215 D). At duplex position 9 in the TRAC off-target \#1 complex, the rU-dG mismatch is 216 accommodated by wobble base-pairing enabled by a minor groove displacement of the dG base 217 (Figure S6E). At the same duplex position in the AAVS1 off-target \#1 and \#2 complexes, 218 however, this mispair occurs next to $\mathrm{rC}-\mathrm{dA}$ and $\mathrm{rC}-\mathrm{dT}$ mismatches, respectively, and adopts the 219 sterically prohibited Watson-Crick geometry (Figure S6F-G), implying a tautomeric shift or 
base deprotonation to accommodate this otherwise unfavorable base pairing mode (Figure

221 S6H). Collectively, these observations suggest that the ability of rU-dG (and likely rG-dT) local sequence context and/or the presence of neighboring mismatches. base is protonated at the N1 position (Garg and Heinemann, 2018; Wang et al., 2011). In the rA-dC mispairs found at duplex position 4 in the FANCF off-target \#2 and \#3 complexes, the $\mathrm{dC}$ nucleotide undergoes a wobble displacement compatible with the formation of two hydrogen bonds with adenine base, indicative of adenine protonation (Figure 2B, Figure S7A). At other duplex positions in our data set, the $\mathrm{rA}-\mathrm{dC}$ or $\mathrm{rC}-\mathrm{dA}$ mispairs are instead accommodated by slight displacements of the adenine base within the base pair plane resulting in the formation of a single hydrogen bond in each case (Figure S7B-D). interactions, rG-dA and $\mathrm{rA}-\mathrm{dG}$ mispairs are accommodated by anti-to-syn isomerization of the adenine base to form two hydrogen-bonding interactions via its Hoogsteen base edge. This is observed at duplex position 11 in the AAVS1 off-target \#4 complex (rG-dA mispair) (Figure 2C) and at position 7 in the $A A V S 1$ off-target \#2 complex (rA-dG mispair) (Figure S7E).

240 Similarly, the rG-dG mispair at duplex position 13 in the FANCF off-target \#5 complex is 241 accommodated by Hoogsteen base-pairing as a result by anti-to-syn isomerization of the guide 242 RNA base (Figure 2D). Overall, the observed Hoogsteen base pairing interactions are near243 isosteric with Watson-Crick base pairs and maintain duplex width without excessive backbone 244 distortion (Table S4). 
Taken together, the prevalence of non-canonical base-pairing interactions, such as wobble and Hoogsteen base pairing, in off-target structures indicates that they serve a fundamental role in off-target recognition. These interactions preserve hydrogen bonding between guide RNA and off-target DNA bases while simultaneously maintaining the integrity of the guide RNA-off-target DNA heteroduplex and minimizing its structural distortions.

Duplex backbone rearrangements accommodate otherwise non-permissive base mispairs

251 Whereas wobble (G-U/T or A-C) and Hoogsteen (A-G or G-G) base pairs are generally compatible with the canonical A-form geometry of an RNA-DNA duplex, other nucleotide mismatches only form non-isosteric base pairs that require considerable distortion of the (deoxy)ribose-phosphate backbone. The formation of pyrimidine-pyrimidine base pairs is expected to occur by a substantial reduction in duplex width. This is observed at the rU-dC 256 mismatch at duplex position 9 in the $F A N C F$ off-target \#1 complex (Figure 3A). Here, the 257 guide RNA backbone is able to shift towards the target DNA strand, resulting in a reduction of 258 the $C 1^{\prime}-\mathrm{C} 1$ ' distance to $8.65 \AA$ as compared to $10.0 \AA$ in the $F A N C F$ on-target complex. This 259 facilitates the formation of two hydrogen bonding interactions within the rU-dC base pair, 260 which is further enabled by a substantial increase in base propeller twist (Figure 3A). In 261 contrast, rC-dT mismatches remain unpaired at duplex positions 6 and 7 in FANCF off-target 262 \#6 and \#7 complexes (Figure S8A-B), respectively, or form only a single hydrogen bond at 263 position 8 in the AAVS1 off-target \#2 complex (Figure S8C), likely due to backbone steric 264 constraints at these positions imposed by Cas9 interactions. Of note, the FANCF off-target \#7 265 rC-dT mispair is bridged by hydrogen bonding interactions with the side chain of Arg895 266 inserted into the minor groove of the heteroduplex (Figure S8B); however, the interaction is 267 not essential for the tolerance of rC-dT mismatches at this position (Figure S9). Backbone steric 268 constraints also likely influence the formation of rU-dT base pairs. At duplex position 7 in the 269 TRAC off-target \#2 complex, the mismatch remains unpaired (Figure S8D), whereas 
productive pairing is seen at duplex positions 8 (PTPRC-tgt2 off-target \#1 complex) and 9 (FANCF off-target \#5 and TRAC off-target \#2 complexes), facilitated by distortions of the guide water molecule or when one of the cytosine bases is protonated (Leontis et al., 2002). Only the former is observed in the determined structures, at duplex position 5 in the AAVS1 off-target \#2 complex (Figure S8G). In contrast, at duplex position 8 and 15 in the AAVS1 off-target \#3 complex, the bases remain unpaired while maintaining intra-strand base stacking interactions

(Figure 3C, Figure S8H). Similarly, rA-dA mispairs are unable to form productive hydrogenbonding interactions within the constraints of an A-form duplex (Leontis et al., 2002). Accordingly, the rA-dA mispair at duplex position 5 in the PTPRC-tgt 2 off-target \#1 complex is accommodated by extrusion of the $\mathrm{dA}$ nucleobase out of the base stack into the major groove of the duplex (Figure 3D). As the duplex width is constrained at this position by Cas9, the base extrusion is enabled by local distortion of the TS backbone (Figure S10A). Analysis of our SITE-Seq assay data set revealed that off-target rA-dA mispairs occur at all positions within the guide RNA-TS DNA heteroduplex (Figure S10B), in agreement with previous studies 286 (Boyle et al., 2017; Boyle et al., 2021; Doench et al., 2016; Jones et al., 2020; Zhang et al., 2020). This suggests that rA-dA mismatches do not encounter steric barriers within Cas 9 that would disfavour their presence, which is consistent with the absence of specific contacts with Cas9 along the length of the major groove of the guide RNA-TS DNA duplex. Collectively, these structural findings indicate that conformational rearrangements of 291 the (deoxy)ribose-phosphate backbone of the guide RNA or TS DNA facilitate interactions of base mispairs that would otherwise be incompatible with canonical A-form duplex geometry.

293 The specific mechanism of base mismatch accommodation at a given position is governed by 
294 local steric constraints on duplex width and the ability of the guide RNA or TS DNA to undergo

295 backbone distortions, which are in turn dictated by local interactions with Cas9.

PAM-proximal mismatches are accommodated by TS distortion due to seed sequence

297 rigidity

298 The seed sequence of the guide RNA (nucleotides 11-20) makes extensive interactions with 299 Cas9, both in the absence and presence of bound DNA (Anders et al., 2014; Jiang et al., 2015; 300 Nishimasu et al., 2014; Zhu et al., 2019). Structural pre-ordering of the seed sequence by Cas9 301 facilitates target DNA binding and contributes to the specificity of on-target DNA recognition 302 (Jiang et al., 2015; O'Geen et al., 2015; Wu et al., 2014). Conversely, binding of off-target 303 DNAs containing PAM-proximal mismatches is inhibited and results in accelerated off-target 304 dissociation (Boyle et al., 2017; Boyle et al., 2021; Ivanov et al., 2020; Jones et al., 2020; Singh 305 et al., 2016; Zhang et al., 2020). Nevertheless, Cas9 does tolerate most base mismatch types 306 within the seed region of the guide RNA, leading to detectable off-target DNA cleavage (Boyle et al., 2021; Doench et al., 2016; Jones et al., 2020; Zhang et al., 2020). In particular, the first two PAM-proximal positions display a markedly higher tolerance for mismatches than the rest of the seed region (Cofsky et al., 2021; Doench et al., 2016; Hsu et al., 2013; Mekler et al., 310 2017; Zeng et al., 2018); this is supported by our SITE-Seq assay data as the frequency of mismatches at the first three PAM-proximal positions is roughly twice as high as at the other seed sequence positions (Figure S1D-E). nucleotides are not directly contacted by Cas 9 in the pre-cleavage state and are thus under fewer 315 steric constraints, with the exception of duplex position 20 in which the phosphodiester group of the TS nucleotide makes extensive interactions with the phosphate lock loop of Cas9 (Anders et al., 2014) (Figure S11A). In agreement with this, our off-target complex structures reveal 
TS backbone, while the conformation of the guide RNA backbone and base stacking within the seed region remain unperturbed. The presence of an rA-dA mismatch in the PAM-proximal position 18 of FANCF off-target \#6 results in the extrusion of the TS nucleobase into the major groove (Figure 4A), likely due to steric constraints on duplex width at this position. In contrast, the rA-dA mismatch at duplex position 19 in the AAVS1 off-target \#2 is instead accommodated by a marked distortion in the TS backbone that results in increased duplex width, which preserves base stacking within the duplex in the absence of productive pairing between the adenine bases (Figure 4B, Figure S11B). Similarly, the rA-dG mismatch at position 19 in the AAVS1 off-target \#5 is accommodated by a $\sim 2 \AA$ displacement of the TS backbone, increasing duplex width. This not only preserves base stacking but also facilitates rA-dG base paring by two hydrogen bonding interactions via their Watson-Crick edges (Figure 4D, Figure S11C).

This off-target complex also contains a rU-dG mispair at duplex position 20 which does not undergo wobble base pairing as the rU20 nucleotide is extensively contacted by Cas 9 and unable to shift towards the major groove and is instead accommodated by a slight shift in the $\mathrm{dG}$ nucleotide (Figure 4D). Finally, the rU-dT base mismatch at duplex position 20 in the AAVS1 off-target \#4 complex remains unpaired and the dT base lacks ordered electron density

(Figure 4C). This is likely a result of the dT nucleotide maintaining contact with the phosphatelock loop of Cas9, which prevents a reduction in the duplex width and precludes productive 337 base pairing.

Overall, these observations indicate that off-target DNAs containing mismatches to the seed sequence of the guide RNA can be accommodated by Cas 9 due to limited interactions with 340 the TS DNA in the seed-binding region, which permits structural distortions of the TS backbone 341 to accommodate base mispairs without steric hindrance and may facilitate non-canonical base 342 pairing interactions. Conversely, the extensive interactions of Cas9 with the ribose-phosphate 
343 backbone of the seed region of the guide RNA provide strong steric constraints that would be

344 expected to disfavour specific base mispairs.

345 Cas9 recognizes off-targets with single-nucleotide deletions by base skipping or via 346 multiple mismatches

347 A substantial fraction of bona fide off-target sites recovered in our SITE-Seq assay analysis 348 (46.4\%, when not considering the possibility of nucleotide insertions or deletions) contained 349 six or more mismatched bases to the guide RNA (Figure S1C, Table S1, Table S2). Such off350 target sequences have previously been proposed to be accommodated by bulging out or 351 skipping of nucleotides (Boyle et al., 2021; Cameron et al., 2017; Doench et al., 2016; Jones et 352 al., 2020; Lin et al., 2014; Tsai et al., 2015), which would result in a shift of the nucleotide 353 register to re-establish correct base pairing downstream of the initially encountered mismatch.

354 The PTPRC-tgt2 off-target \#1, FANCF off-target \#3 and AAVS1 off-target \#2 sites are predicted 355 to contain single nucleotide deletions at duplex positions 15, 17 and 9, respectively (Figure 1A, 356 Figure 5C). Structures of the PTPRC-tgt2 off-target \#1 and FANCF off-target \#3 complexes 357 reveal that the single nucleotide deletions in these off-target substrates are not accommodated 358 by bulging out the unpaired guide RNA nucleotide. Instead, the conformations of the guide 359 RNAs remain largely unperturbed and the off-target TS DNAs "skip over" the unpaired RNA 360 bases to resume productive base-pairing downstream (Figure 5A-B). Comparisons with the $361 F A N C F$ on-target complex structure show that the seed sequences of the guide RNAs are held 362 in place by interactions with the bridge helix and the REC1 domain, whereas the DNA target 363 strand phosphate backbones are displaced by almost $3 \AA$ (Figure S12A-B). The base pair skips 364 are accommodated by considerable buckling and tilting of the base pairs immediately 365 downstream of the skip site. An additional consequence of the base pairing register shift is the 366 formation of non-canonical base pairs between the off-target DNA and the extra 5'-terminal 367 guanine nucleotides present in the guide RNA as a consequence of in vitro transcription by T7 
RNA polymerase (Figure S12C-D). This potentially explains the impact of the 5'-guanines on both R-loop stability and in vitro cleavage activity (Kulcsar et al., 2020; Mullally et al., 2020; Okafor et al., 2019).

Originally, our SITE-Seq assay analysis annotated the AAVS1 off-target \#2 as a singlenucleotide deletion at duplex position 9 (Figure 5C). Unexpectedly, the structure of the AAVS1 off-target \#2 complex instead reveals that the off-target substrate is bound in the unshifted register, resulting in the formation of five base mismatches in the PAM-distal half of the guide RNA-TS duplex (Figure 5D), including a partially paired $\mathrm{rC}-\mathrm{dC}$ mismatch at position 5, an rA-dG Hoogsteen pair at position 7, a partially paired $\mathrm{rC}-\mathrm{dT}$ mismatch at position 8 , and a tautomeric $\mathrm{rU}-\mathrm{dG}$ pair at position 9. The backbone conformations of the guide RNA and the 378 off-target TS exhibit minimal distortions and are nearly identical with the corresponding on379 target heteroduplex (Figure 5D), suggesting an explanation for the tolerance of the multiple 380 mismatches in this off-target site, and implying that certain mismatch combinations might 381 cumulatively result in guide RNA and TS backbone conformations that mimic the on-target 382 situation. To test this hypothesis, we reverted the $\mathrm{rC}$-dT mismatch at position 8 to the on-target 383 rC-dG pair, thereby reducing the total amount of off-target mismatches from 6 to 5 (Figure 384 5C). The resulting off-target substrate (AAVS1 off-target \#6) exhibited substantially reduced 385 cleavage rates in both dsDNA and PAMmer formats, as well as a significantly increased 386 dissociation rate (Figure 5E-F). These results suggest that for some bona fide off-target 387 substrates containing mismatch combinations, the reversal of one mismatch may affect the 388 structural integrity of the guide RNA-TS DNA heteroduplex and interfere with DNA binding 389 and/or conformational activation of Cas9, despite a reduction in the total number of 390 mismatches.

391 Collectively, these results indicate that deletion-containing off-target complexes are 392 accommodated either by RNA base skipping, as opposed to RNA nucleotide bulging, or by the 
393

formation of multiple base mismatches. The precise mechanism appears to be dependent on the

394 position of the deletion. Because the seed sequence nucleotides 12-20 of the guide RNA are 395 extensively contacted by Cas9 (Figure S13), while the complementary DNA nucleotides are 396 able to undergo distortions to accommodate a shift in the base pairing register, deletions at positions within the PAM-proximal region of the guide RNA-TS heteroduplex (positions 1120) result in RNA base skipping. In contrast, deletions at PAM-distal positions (1-10), where the guide RNA-TS DNA heteroduplex is constrained by interactions with the REC3 and HNH domains, are instead likely to be bound without a register shift via multiple mismatches.

In light of our structural findings, we computationally analyzed off-target sites identified by the SITE-Seq assay for the presence of insertions or deletions in the target DNA relative to the guide RNA sequence. Our initial off-target classification algorithm assumed that deletions and insertions can occur along the entire guide RNA-off-target DNA heteroduplex. Based on our structural data we subsequently constrained the algorithm to only consider singlenucleotide deletions and insertions at heteroduplex positions $10-20$ and 6-20, respectively (Figure S14). This resulted in a substantial reduction in the number of off-targets containing deletions (from 323 to 277) but no change in off-targets predicted to contain insertions (116 sites for both). When extrapolated, these results collectively suggest that up to $14 \%$ of off-target sites previously annotated as containing deletions or insertions in off-target studies might instead be recognized via multiple mismatches (Figure S15), which has implications for offtarget prediction, as discussed below.

414 FANCF off-target \#4, which contains three PAM-distal mismatches at positions 1-3 and a G-U 415 mismatch in position 10 (Figure 1A), is reproducibly the top ranking off-target site for the 416 FANCF guide RNA, as detected by SITE-Seq assay analysis at the lowest Cas9 RNP 417 concentrations (Table S1, Table S2). The off-target substrate exhibits slow cleavage kinetics 
in vitro with both dsDNA and PAMmer substrates (Figure 2B, Figure S2A-B), indicating a perturbation of the conformational activation checkpoint of Cas9. The structure of the FANCF off-target \#4 complex reveals that the RNA-DNA heteroduplex is unpaired at positions 1-3 as a result of the PAM-distal mismatches, with nucleotides 1-2 of the guide RNA and 19-20 of the TS disordered (Figure 6A). Furthermore, Cas9 undergoes structural rearrangements of its REC lobe and the HNH domain (Figure 6B), resulting in a root mean square displacement of the REC2 and REC3 domains of $3.7 \AA(1,315 \mathrm{C} \alpha$ atoms) relative to the $F A N C F$ on-target complex structure. The REC3 domain undergoes a 19-degree rotation (Figure 6B), facilitated by extending the helix comprising residues 703-712 through restructuring of loop residues 713716 (Figure S16A), to accommodate the altered guide RNA conformation. The REC2 domain rotates 32 degrees away from the REC3 domain (Figure 6B). This is accompanied by restructuring of the hinge loop residues 174-180 and disordering of loops 258-264, 284-285, and 307-309. Concomitantly, the HNH domain rotates $11^{\circ}$ away from the heteroduplex, as compared to the $F A N C F$ on-target structure, to accommodate distortion of the TS DNA (Figure 6B).

The unpaired 5' end of the sgRNA is located at the interface between the REC3 and the 434 RuvC domain and maintains interactions with heteroduplex-sensing residues Lys510, Tyr515, and Arg661 of the REC3 domain (Figure S16B). In contrast to the corresponding on-target 436 complex structure, the unpaired 3' end of the off-target TS breaks away from the REC3 lobe 437 and instead points towards the REC2 domain, forming unique interactions with Arg895, 438 Asn899, Arg905, Arg919 and His930 in the HNH domain (Figure S16C). These interactions 439 (Figure S16D) could be responsible for the observed repositioning of the REC lobe and HNH 440 domain, and they may impede the formation of a cleavage-competent complex. The conformation of the FANCF off-target \#4 complex is distinct from the 442 conformations observed in cryo-EM reconstructions of the pre- and post-cleavage states of the 
443 Cas9 complex (Zhu et al., 2019) (Figure S17A-B). Instead, the off-target complex structure 444 most closely resembles that of a high-fidelity variant xCas9 3.7 containing amino acid 445 substitutions that disrupt interactions with the TS DNA (Guo et al., 2019). Although the xCas9

4463.7 complex adopts a slightly different REC lobe conformation (Figure S17C), the PAM-distal 447 duplex also undergoes unpairing at positions 1-3 and displays a comparable degree of structural 448 disorder (Figure S17D). These structural observations thus suggest that the presence of 449 multiple mismatches in the PAM-distal region of a guide RNA-off-target DNA duplex leads to 450 conformational perturbations in the DNA-bound complex that resemble the structural 451 consequences of specificity-enhancing mutations in high-fidelity Cas9 variants. 


\section{Discussion}

455 The off-target activity of Cas9 has been extensively documented in prior genome editing, 456 biochemical and biophysical studies (Boyle et al., 2017; Boyle et al., 2021; Doench et al., 2016; 457 Jones et al., 2020; Lazzarotto et al., 2020; Zhang et al., 2020). Although numerous methods 458 have been devised for computational prediction of genomic off-target sites and their 459 experimental validation, these have reported highly variable mismatch tolerance profiles 460 depending on the screening method and the target sequence. Thus, a comprehensive 461 understanding of this phenomenon is still lacking, particularly as to whether off-target tolerance 462 has an underlying structural basis. In this study, we used the SITE-Seq assay to examine the 463 off-target landscape of 12 well-studied guide RNAs, observing a broad variation of cleavage 464 activities associated with individual off-target substrates. To shed light on the molecular mechanisms underpinning off-target activity, we determined atomic structures of a 466 representative set of bona fide off-target complexes, thus providing fundamental insights into 467 the structural aspects of off-target recognition.

468 Role of non-canonical base pairing in off-target recognition

469 The principal, and largely unexpected, finding of our structural analysis is that the majority of 470 nucleotide mismatches in bona fide off-target substrates are accommodated by non-canonical 471 base pairing interactions. These range from simple $\mathrm{rG}-\mathrm{dT} / \mathrm{rU}-\mathrm{dG}$ wobble or Hoogsteen base 472 pairing interactions, to pyrimidine-pyrimidine pairs that rely on (deoxy)ribose-phosphate 473 backbone distortions that reduce duplex width. With the notable exception of rA-dA mispairs, 474 which are accommodated at certain positions within the guide-TS heteroduplex by base 475 extrusion, the structural rearrangements associated with base mismatch accommodation 476 preserve base stacking, which is the primary determinant of nucleic acid duplex stability 477 (Yakovchuk et al., 2006). For some off-target sequences, our structures are suggestive of base 478 protonation or tautomerization, which facilitate hydrogen bonding interactions in otherwise 
non-permissive base pair combinations, such as $\mathrm{rA}-\mathrm{dC}$. These rare base pair forms have been

480 previously observed in both RNA and DNA duplexes and are thought to be important 481 contributors to DNA replication and translation errors (Kimsey et al., 2015; Kimsey et al., 482 2018). Future studies employing complementary structural methods, such as nuclear magnetic 483 resonance, will help confirm the occurrence of non-canonical base states in off-target 484 complexes. does not directly contact the major- or minor-groove edges of the guide RNA-TS DNA heteroduplex base pairs at any of the duplex positions and thus lacks a steric mechanism to enforce Watson-Crick base pairing. This is further underscored by Cas9's tolerance of base modifications in target DNA, including cytosine 5-hydroxymethylation and, at least at some 490 duplex positions, glucosyl-5-hydroxymethylation (Vlot et al., 2018). In this respect, Cas9 491 differs from other molecular systems, notably the ribosome and replicative DNA polymerases, 492 which enhance the specificity of base-pairing by direct readout of base-pair shape and steric 493 rejection of mispairs (Kunkel and Bebenek, 2000; Rodnina and Wintermeyer, 2001; Timsit, 494 1999). Secondly, Cas9 is a multidomain protein that displays considerable conformational 495 dynamics and is therefore able to accommodate local distortions in the guide-TS duplex 496 geometry by compensatory rearrangements of the REC2, REC3 and HNH domains. Indeed, in 497 most off-target structures reported in this study, almost all atomic contacts between Cas9 and 498 the guide-TS heteroduplex are preserved. Thus, Cas9 only detects guide-target mismatches by 499 indirect readout of the guide RNA-TS DNA heteroduplex width, except at the PAM-distal end 500 of the heteroduplex where base mismatches result in duplex unpairing, as discussed below. Our 501 observations are consistent with recent molecular dynamics simulation studies showing that 502 internally positioned mismatches within the guide RNA-TS DNA heteroduplex are readily 503 incorporated within the heteroduplex and have only minor effects on Cas9 interactions 
504 (Mitchell et al., 2020). The lack of a steric base-pair enforcement mechanism and the resulting

505 off-target promiscuity likely reflects the biological function of Cas9 in CRISPR immunity, 506 where mismatch tolerance contributes to interference by enabling the targeting of closely 507 related viruses and hindering immune evasion by mutations or covalent base modifications 508 (Deveau et al., 2008; Semenova et al., 2011; van Houte et al., 2016; Yaung et al., 2014).

509 Structural rigidity of the guide RNA seed region and implications for off-target 510 recognition

511 The seed sequence of the Cas9 guide RNA (nucleotides 11-20) is the primary determinant of 512 target DNA binding, a consequence of its structural pre-ordering in an A-like conformation by 513 extensive interactions with Cas9 (Anders et al., 2014; Jiang et al., 2015; Nishimasu et al., 2014; 514 Zhu et al., 2019). Our data indicate that structural rigidity of the guide RNA seed sequence also 515 affects off-target recognition, as base mispairs in the seed region of the guide-off-target 516 heteroduplex can only be accommodated by conformational distortions of the TS DNA, which 517 is subject to only a few steric constraints, notably at position 20 due to interactions with the 518 phosphate lock loop (Anders et al., 2014). The rigidity of the guide RNA seed sequence 519 increases the energetic penalty of base mispairing in the seed region of the heteroduplex, and 520 thus contributes to mismatch sensitivity of Cas9 within the seed region. Although structural 521 distortions of TS DNA facilitate biding of off-target substrates containing seed mismatches, 522 they may nevertheless inhibit off-target cleavage by steric hindrance of the HNH domain, 523 thereby further contributing to the general mismatch intolerance of the guide RNA seed 524 sequence. The contrasting structural plasticities of the guide RNA and TS DNA strands are 525 manifested in the differential activities of Cas9 against off-targets containing rU-dG and rG-dT 526 mismatches within the seed region (Boyle et al., 2021; Doench et al., 2016; Hsu et al., 2013; 527 Jones et al., 2020; Zhang et al., 2020). Whereas rG-dT mismatches can be readily 528 accommodated by wobble base pairing, seed sequence rigidity is expected to hinder rU-dG 
wobble base pairing. Combined with a lower energetic penalty associated with rG-dT mismatch

530 binding (binding an off-target with an rG-dT mismatch requires unpairing a dT-dA base pair in

531 the off-target DNA, while rU-dG off-target recognition requires dC-dG unpairing), these effects

532 thus help Cas9 discriminate against rU-dG mismatches in the seed region.

\section{Recognition of off-targets containing insertions and deletions}

Bona fide off-target sites containing insertions or deletions have been detected in a number of studies (Boyle et al., 2021; Cameron et al., 2017; Doench et al., 2016; Jones et al., 2020; Tsai et al., 2015). Nucleotide "bulging" has been proposed as a mechanism to recognize such an offtarget, which would otherwise result in a large number of consecutive base mismatches. However, as Cas9 encloses the guide RNA-TS DNA heteroduplex in a central channel and makes extensive interactions along the entire length of the guide RNA strand, the formation of RNA bulges is precluded due to steric clashes, pointing to a different mechanism. Indeed, the structures of PTPRC-tgt2 off-target \#1 and FANCF off-target \#3 complexes reveal that off-target sequences predicted to contain single-nucleotide deletions in the seed region of the heteroduplex are instead recognized by base skipping, resulting in an unpaired guide RNA base within the duplex stack. Due to the lack of extensive contacts of Cas9 with the TS and the rigid coordination of the guide RNA in the seed region, these findings suggest that single nucleotide deletions can only be accommodated within the seed region of the heteroduplex and not elsewhere. This is supported by the observation that the AAVS1 off-target \#2 site, which was previously predicted to contain an RNA bulge or skip in the PAM-distal region (Cameron et al., 2017; Lazzarotto et al., 2020), is recognised via multiple mismatches. Our structural observations indicate that bona fide off-targets predicted to contain single deletions within the seed region of the heteroduplex are recognized by base skipping, which incurs a large energetic penalty. As the seed region of the TS DNA is devoid of Cas9 contacts in the pre-cleavage state (Zhu et al., 2019), off-target sequences containing single-nucleotide 
554 insertions in the seed region of the heteroduplex are likely to be recognized by DNA nucleotide

555 bulging, likewise incurring a large energetic penalty as unwinding an off-target DNA sequence 556 containing an insertion requires breaking an extra base pair. Additionally, TS DNA distortion 557 might inhibit cleavage by steric hindrance of the HNH domain. These observations thus explain 558 why Cas9 appears to tolerate mismatches better than insertions or deletions (Boyle et al., 2021; 559 Cameron et al., 2017; Doench et al., 2016; Jones et al., 2020) and why deletions and insertions 560 within the seed region are particularly deleterious. In contrast, off-target sequences containing 561 insertions or deletions in the PAM-distal region of the heteroduplex, where both the guide RNA 562 and TS DNA strands are contacted by Cas9, are instead likely to be bound in the unchanged 563 register, with multiple base mispairs accommodated by non-canonical base pairing interactions. 564 Our analysis suggests that a significant fraction of off-target sites previously predicted to 565 contain insertions or deletions may be recognized in this manner.

PAM-distal base pairing and the conformational checkpoint of Cas9

567 Upon substrate DNA hybridization and R-loop formation, Cas9 undergoes conformational 568 activation of its nuclease domains (Zhu et al., 2019). The Cas9 REC3 domain plays a key role 569 in the process, as it senses the integrity of the PAM-distal region of the guide RNA-TS DNA 570 heteroduplex and allosterically regulates the REC2 and $\mathrm{HNH}$ domains, providing a 571 conformational checkpoint that traps Cas9 in a conformationally inactive state in the absence 572 of PAM-distal hybridization (Chen et al., 2017; Dagdas et al., 2017; Palermo et al., 2018; Zhu 573 et al., 2019). Our structural data confirm that mismatches at the PAM-distal end of the 574 heteroduplex (positions 1-3) result in heteroduplex unpairing, incomplete R-loop formation and 575 structural repositioning of the REC3 domain, indicating a perturbation of the Cas9 576 conformational checkpoint. We envision that the observed conformational state mimics the 577 structural effect of 5'-truncated guide RNAs, which have been shown to improve targeting 578 specificity (Fu et al., 2014). Furthermore, similarities with the structure of a high-fidelity Cas9 
variant (Guo et al., 2019) suggest a shared underlying mechanism for increased specificity. In

580 both cases, disruption of REC3 contacts with the PAM-distal heteroduplex modulates REC2/3

581 domain positioning, hindering allosteric activation of the $\mathrm{HNH}$ nuclease domain (Chen et al.,

582 2017; Dagdas et al., 2017; Palermo et al., 2018). This is also consistent with observations that

583 REC2/3 domain repositioning in Cas9 complexes with chimeric RNA-DNA guides modulates

584 cleavage efficiency and results in increased specificity by slowing down conformational

585 nuclease activation and promoting substrate DNA dissociation (Donohoue et al., 2021). In

586 addition, the establishment of new HNH protein contacts with the heteroduplex, as observed in

$587 F A N C F$ off-target \#4, has been proposed to affect the active site positioning of the HNH domain

588 (Mitchell et al., 2020; Ricci et al., 2019; Zeng et al., 2018). Indeed, it has been demonstrated

589 that truncated guides result in reduced cleavage rates due to impaired HNH docking (Dagdas et

590 al., 2017).

591 Implications for off-target prediction

592 Our structural data reveal that Cas9 plays a limited steric role in off-target discrimination insofar

593 as only sensing the integrity and general shape of the guide-target heteroduplex. Off-target

594 activity is thus largely determined by the kinetics and energetics of R-loop formation, i.e., off-

595 target DNA strand separation and guide RNA-TS DNA hybridization, and the Cas9

596 conformational activation checkpoint. We observe on multiple occasions in the determined off-

597 target complexes that a given base mismatch adopts different conformational arrangements

598 depending on its position along the guide RNA-TS DNA heteroduplex. This poses a challenge

599 for ab initio modelling of off-target activity, as biophysical models of off-target binding and

600 cleavage are bound to be of limited accuracy unless they incorporate position-dependent

601 energetic penalties for each base mismatch type and for deletions, as well as position- and base-

602 specific penalties for insertions (Boyle et al., 2021; Jones et al., 2020; Zhang et al., 2020). In

603 addition, as certain off-target sequences that are incompatible with dsDNA cleavage can 
604 undergo NTS nicking (Fu et al., 2019; Jones et al., 2020; Murugan et al., 2020; Zeng et al., 605 2018), future bioinformatic models need to be able to predict off-target nicking activity as well.

606 Furthermore, accurate modelling of off-target interactions remains difficult due to context607 dependent effects, as documented in previous studies showing that the binding and cleavage 608 defects of consecutive mismatches deviate from additivity (Boyle et al., 2021; Cameron et al., 609 2017; Lazzarotto et al., 2020; Zhang et al., 2020). Indeed, our structural data rationalize this by 610 showing that the conformation of a given base mismatch is highly sensitive to the presence of 611 neighbouring mismatches. As seen in the case of AAVS1 off-target \#2 complex, multiple 612 mismatched bases can synergistically combine to preserve an on-target-like heteroduplex 613 conformation that passes the REC3 conformational checkpoint, supporting nearly on-target 614 efficiencies of cleavage (Zhang et al., 2020). This is in line with recent cryo-EM structural 615 studies suggesting that indirect readout of heteroduplex conformation is coupled to nuclease 616 activation, while the presence of mismatches disrupts this coupling (Bravo et al., 2021; Pacesa 617 and Jinek, 2021). Critically, reversion of one of the mismatches in this off-target substrate 618 impairs cleavage activity. Similar effects have been described for other DNA binding proteins 619 such as transcription factors, where mismatches modulate the binding activity of the protein by 620 affecting the conformation of the DNA duplex (Afek et al., 2020). In an analogy with Cas9, 621 these proteins check for correct binding sites through indirect sequence readout by sampling 622 for the correct duplex shape rather than base sequence (Abe et al., 2015; Kitayner et al., 2010; 623 Rohs et al., 2009a; Rohs et al., 2009b). In conclusion, structural insights presented in this study establish an initial framework 625 for understanding the molecular basis for the off-target activity of Cas9. In conjunction with 626 ongoing computational studies, these findings will help achieve improved energetic 627 parametrization of off-target mismatches and deletions/insertions, thus contributing to the 628 development of more accurate off-target prediction algorithms and more specific guide RNA 
629 designs. In doing so, these studies will contribute towards increasing the precision of CRISPR-

630 Cas9 genome editing and the safety of its therapeutic applications.

631 
632

633

634

635

636

637

638

639

640

641

642

643

644

646

647

648

649

650

651

652

653

654

655

\section{Author contributions}

M.P., P.C., P.D.D., and M.J. conceived the study. M.P. purified wild-type Cas9, performed in vitro cleavage assays, crystallized ternary Cas9 complexes, solved the structures, and performed structural analysis along with M.J.; A.C. performed switchSENSE binding measurements, under the supervision of F.H.T.A; M.J.I. performed the SITE-Seq assay; C-H.L. wrote the computational off-target classification model and P.D.D. and P.C. analysed the output; K.B. purified dCas9, transcribed sgRNAs, and prepared DNA substrates for in vitro cleavage assays; M.P., F.H.T.A., P.C., P.D.D., and M.J. wrote the manuscript.

\section{Conflict of interest statement}

P.D.D. and M.J.I are current employees of Caribou Biosciences, Inc., and C-H.L. and P.C. are former employees of Caribou Biosciences, Inc. M.J. is a co-founder of Caribou Biosciences, Inc. M.J., C-H.L., M.J.I., P.C. and P.D.D. are named inventors on patents and patent applications related to CRISPR-Cas technologies.

\section{Acknowledgements}

We thank members of the Jinek laboratory and Caribou Biosciences, Inc. for discussion and critical reading of the manuscript. We thank Vincent Olieric, Meitian Wang, and Takashi Tomizaki (Swiss Light Source, Paul Scherrer Institute) for assistance with crystallographic data collection and Nena Matscheko from Dynamic Biosensors for support with switchSENSE experiments. We thank the NCCR RNA and Disease for providing infrastructural support. This work was supported by the Swiss National Science Foundation Grant 31003A_182567 (to M.J.). M.J. is an International Research Scholar of the Howard Hughes Medical Institute and Vallee Scholar of the Bert L \& N Kuggie Vallee Foundation. 
bioRxiv preprint doi: https://doi.org/10.1101/2021.11.18.469088; this version posted November 18, 2021. The copyright holder for this preprint (which was not certified by peer review) is the author/funder. All rights reserved. No reuse allowed without permission.

\section{$656 \quad$ Figures and Legends}

a
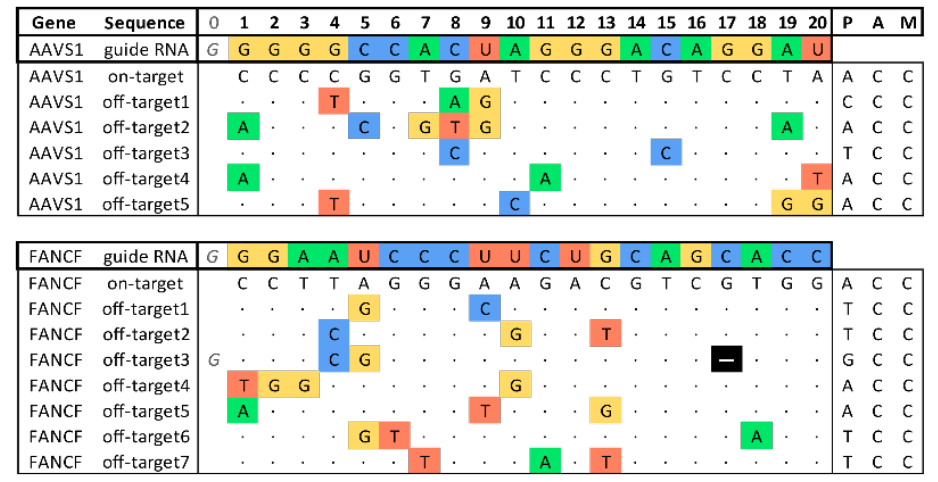

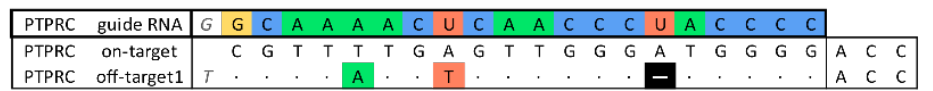

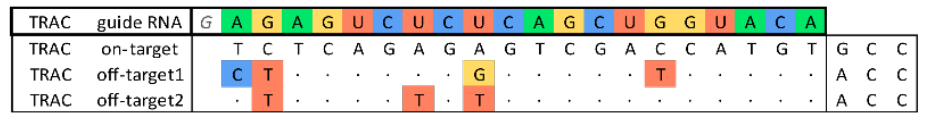

b

\begin{tabular}{|c|c|ccccc|cc|} 
Gene & Target & $\begin{array}{c}(\%) \mathbf{~ 2 4 h} \\
\text { cleavage }\end{array}$ & $\mathbf{k}_{\text {obs }}\left(\mathbf{s}^{-1}\right)$ & $\mathbf{k}_{\text {on }}\left(\mathbf{M}^{-1} \cdot \mathbf{s}^{-1}\right)$ & $\mathbf{k}_{\text {off }}\left(\mathbf{s}^{-1}\right)$ & $\mathbf{k}_{\mathbf{d}}(\mathbf{p M})$ & $\begin{array}{c}\text { 24h cleavage } \\
\text { PAMmer }(\%)\end{array}$ & $\begin{array}{c}\mathbf{k}_{\text {obs }}\left(\mathbf{s}^{-1}\right) \\
\text { PAMmer }\end{array}$ \\
\hline AAVS1 & on-target & 92.2 & 1.6240 & $3.95 \mathrm{E}+06$ & $5.74 \mathrm{E}-05$ & 14.5 & 95.0 & 0.5622 \\
AAVS1 & off-target1 & 92.7 & 0.0713 & $4.73 \mathrm{E}+06$ & $6.29 \mathrm{E}-05$ & 13.3 & 87.5 & 0.2390 \\
AAVS1 & off-target2 & 95.1 & 0.0337 & $8.76 \mathrm{E}+06$ & $3.38 \mathrm{E}-03$ & 386.0 & 85.5 & 0.0040 \\
AAVS1 & off-target3 & 96.7 & 0.0511 & $3.30 \mathrm{E}+06$ & $2.51 \mathrm{E}-03$ & 761.0 & 82.7 & 0.0806 \\
AAVS1 & off-target4 & 94.4 & 0.0039 & $1.09 \mathrm{E}+07$ & $3.28 \mathrm{E}-03$ & 301.0 & 89.6 & 0.0645 \\
AAVS1 & off-target5 & 18.9 & 0.0001 & $\mathrm{ND}$ & $\mathrm{ND}$ & $\mathrm{ND}$ & 70.1 & 0.0068 \\
FANCF & on-target & 97.5 & 0.2383 & $3.45 \mathrm{E}+06$ & $7.46 \mathrm{E}-05$ & 21.6 & 98.3 & 0.5654 \\
FANCF & off-target1 & 35.1 & 0.0006 & $3.97 \mathrm{E}+06$ & $2.09 \mathrm{E}-03$ & 528.0 & 97.4 & 0.0693 \\
FANCF & off-target2 & 62.4 & 0.0006 & $1.42 \mathrm{E}+06$ & $2.45 \mathrm{E}-03$ & 1730.0 & 92.9 & 0.2333 \\
FANCF & off-target3 & 0.0 & 0.0000 & $1.22 \mathrm{E}+07$ & $2.37 \mathrm{E}-03$ & 193.0 & 4.2 & 0.0000 \\
FANCF & off-target4 & 53.0 & 0.0005 & $3.35 \mathrm{E}+06$ & $1.91 \mathrm{E}-03$ & 571.0 & 38.9 & 0.0011 \\
FANCF & off-target5 & 80.4 & 0.0010 & $1.27 \mathrm{E}+06$ & $2.55 \mathrm{E}-03$ & 2010.0 & 92.9 & 0.0584 \\
FANCF & off-target6 & 8.2 & 0.0001 & $1.50 \mathrm{E}+06$ & $2.03 \mathrm{E}-03$ & 1350.0 & 66.6 & 0.0007 \\
FANCF & off-target7 & 5.2 & 0.0036 & $2.95 \mathrm{E}+06$ & $3.21 \mathrm{E}-03$ & 1090.0 & 94.5 & 0.0134 \\
PTPRC & on-target & 96.8 & 0.4588 & $6.08 \mathrm{E}+06$ & $2.19 \mathrm{E}-04$ & 36.0 & 95.5 & 0.0741 \\
PTPRC & off-target1 & 0.0 & 0.0000 & $1.22 \mathrm{E}+07$ & $2.39 \mathrm{E}-03$ & 196.0 & 91.4 & 0.0012 \\
TRAC & on-target & 97.7 & 0.3808 & $1.02 \mathrm{E}+07$ & $3.23 \mathrm{E}-04$ & 31.8 & 93.5 & 0.1812 \\
TRAC & off-target1 & 95.8 & 0.0195 & $1.37 \mathrm{E}+06$ & $1.77 \mathrm{E}-04$ & 130.0 & 90.7 & 0.0807 \\
TRAC & off-target2 & 65.0 & 0.0007 & $9.43 \mathrm{E}+06$ & $3.27 \mathrm{E}-04$ & 34.6 & 88.4 & 0.0260 \\
\hline
\end{tabular}

C
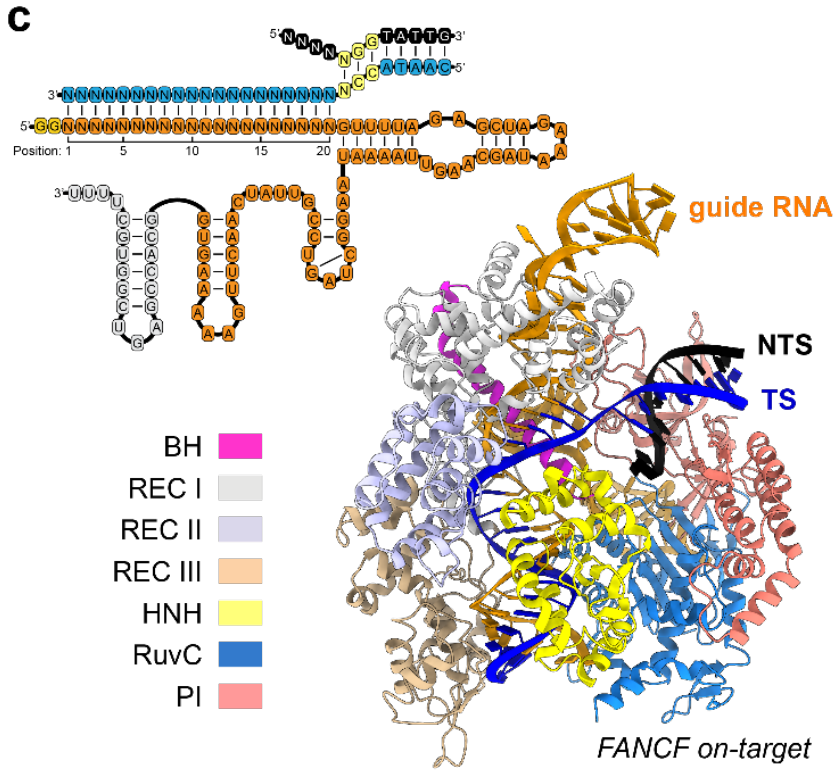
658 Figure 1. Biochemical and structural analysis of Cas9 off-targets.

659 (A) Guide RNA and (off-)target DNA sequences selected for biochemical and structural 660 analysis. Matching bases in off-targets are denoted by a dot; nucleotide mismatches and 661 deletions (-) are highlighted. (B) Kinetic and thermodynamic parameters of off-target 662 substrates. The cleavage rate constants $\left(\mathrm{k}_{\mathrm{obs}}\right)$ were derived from single-exponential function 663 fitting of measured cleavage rates. The binding and dissociation rate constants $\left(\mathrm{k}_{\text {on }}\right.$ and $\left.\mathrm{k}_{\text {off }}\right)$ and 664 the equilibrium dissociation constant $\left(\mathrm{K}_{\mathrm{d}}\right)$ were determined using a DNA nanolever binding 665 (switchSENSE) assay. (C) Top: Schematic representation of the guide RNA (orange), TS 666 (blue), and NTS (black) sequences used for crystallisation. The PAM sequence in the DNA is 667 highlighted in yellow. Bottom: Structure of the Cas9 FANCF on-target complex. Individual 668 Cas9 domains are coloured according to the legend; substrate DNA target strand (TS) is 669 coloured blue, non-target strand (NTS) black, and the guide RNA orange.

670 
A

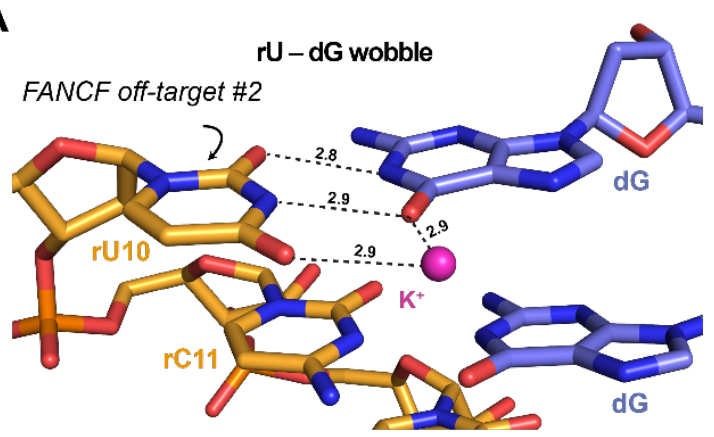

C

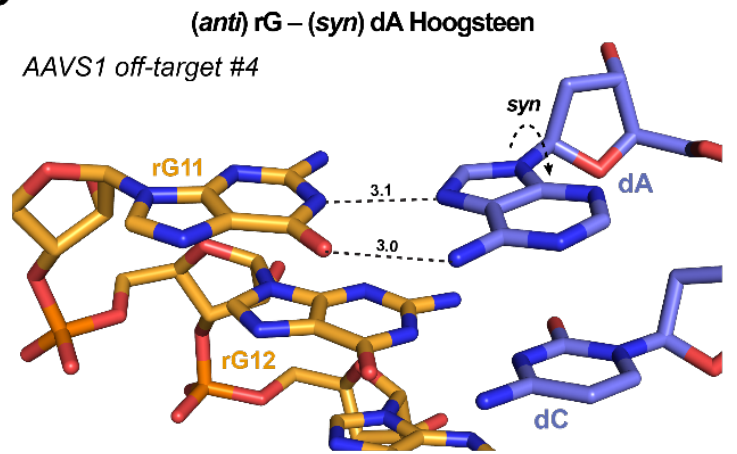

B

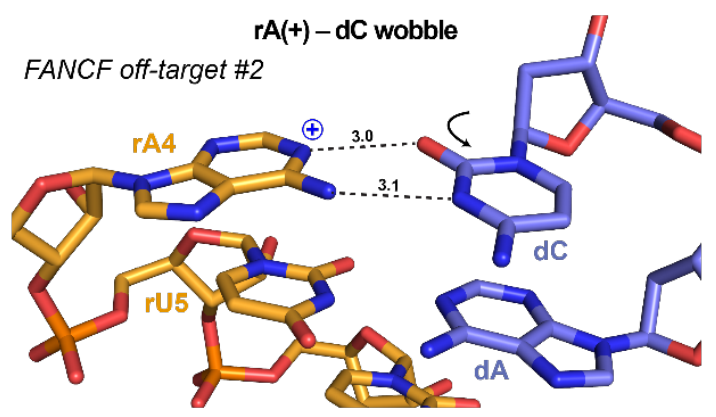

D (syn) rG Hoogsteen - (ant) dG

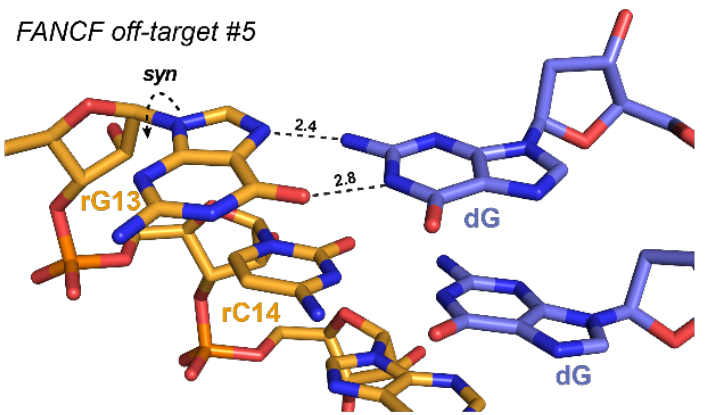

Figure 2. Cas9 off-target binding is enabled by non-canonical base pairing.

673 Close-up views of (A) rU-dG wobble base pair at duplex position 10 in FANCF off-target \#2

674 complex, (B) rA-dC wobble base pair at position 4 in FANCF off-target \#2 complex, (C) rG-

$675 \mathrm{dA}$ Hoogsteen base pair at duplex position 11 in AAVS1 off-target \#4 complex and (D) rG-dG

676 Hoogsteen base pair at duplex position 13 in FANCF off-target \#5 complex. Hydrogen bonding

677 interactions are indicated with dashed lines. Numbers indicate interatomic distances in $\AA$. Solid

678 arrows indicate conformational changes relative to the corresponding on-target complex

679 structures. Dashed arrows indicate anti-syn isomerization of the dA and rG bases to enable

680 Hoogsteen-edge base pairing. A bound monovalent ion, modelled as $\mathrm{K}^{+}$, is depicted as a purple

681 sphere. 
A

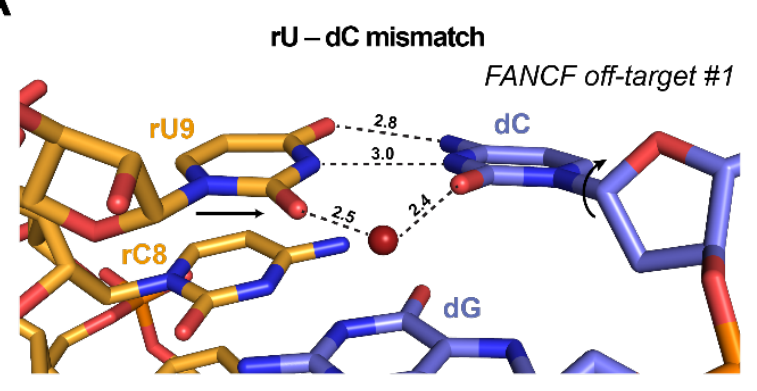

C

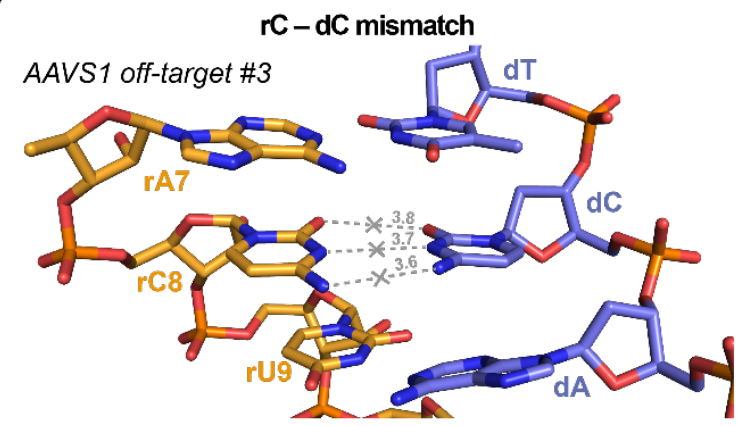

B

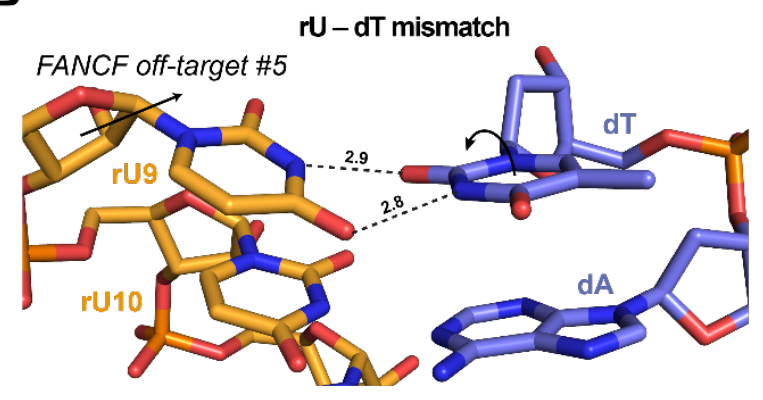

D

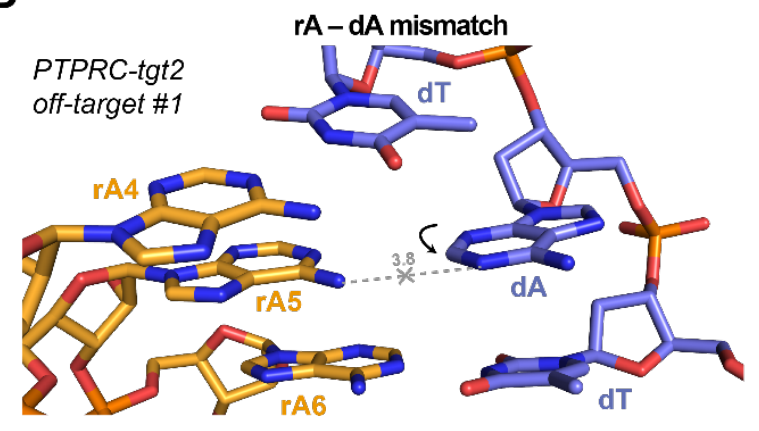

683

684 Figure 3. Duplex backbone distortions facilitate formation of non-canonical base pairs.

685 (A) Close-up view of the rU-dC base pair at duplex position 9 in FANCF off-target \#1 complex, 686 facilitated by lateral displacement of the guide RNA backbone (solid arrow). Hydrogen bonding 687 interactions are indicated with dashed lines. Solid arrows indicate conformational changes 688 relative to the on-target complex. Numbers indicate interatomic distances in $\AA$. Bound water 689 molecule is depicted as red sphere. (B) Zoomed-in view of the rU-dT base pair at position 9 in 690 FANCF off-target \#5 complex. Solid arrows indicate lateral displacement of the rU nucleotide 691 and propeller twist of the dT base. (C) Zoomed-in view of the $\mathrm{rC}-\mathrm{dC}$ mispair at duplex position 6928 in AAVS1 off-target \#3 complex. The distances between the cytosine bases indicate lack of 693 hydrogen bonding. (D) Zoomed-in view of the rA-dA mispair at duplex position 5 in PTPRC694 tgt2 off-target \#1 complex. 
A

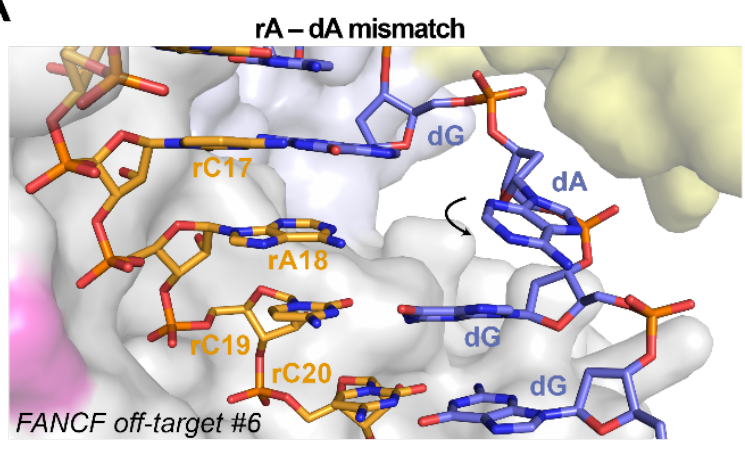

C

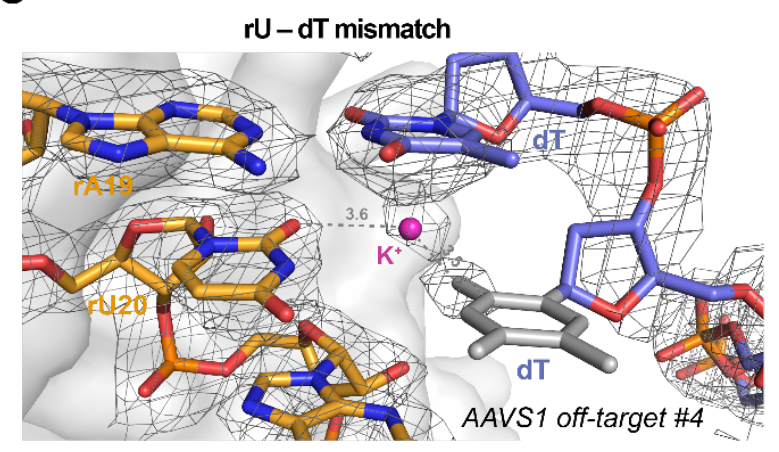

B

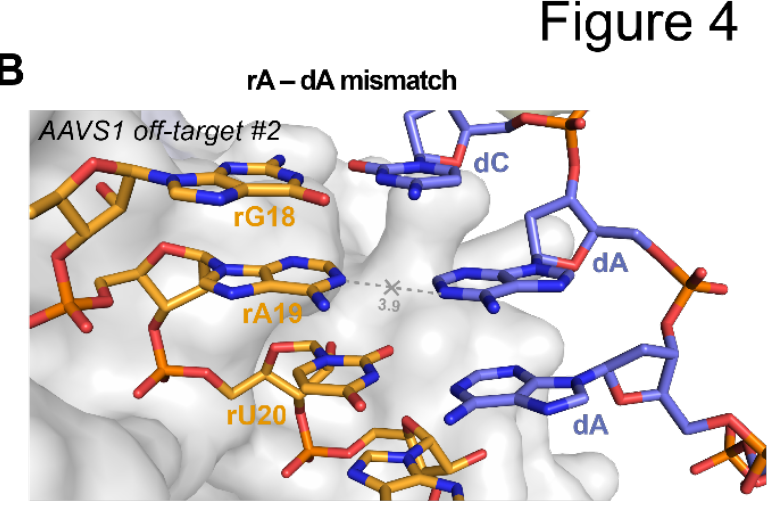

D

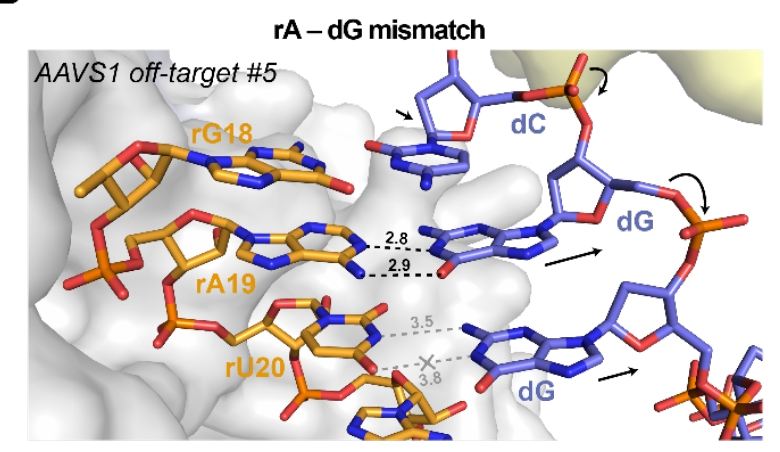

695

696

697

698

699

700

701

702

703

704

705

706

707

708

\section{Figure 4}

Figure 4. TS distortion facilitates mismatch accommodation in the seed region of the guide-off-target heteroduplex.

(A) Close-up view of the rA-dA mismatch at position 18 in FANCF off-target \#6 complex, showing major groove extrusion of the dA base. (B) Close-up view of the rA-dA mismatch at position 19 in AAVS1 off-target \#2 complex, showing retention of the dA base in the duplex stack. (C) Close-up rU-dT mispair at the PAM-proximal position 20 in AAVS1 off-target \#4 complex. Residual electron density indicates the presence of an ion or solvent molecule. Refined $2 \mathrm{~m} F_{\mathrm{o}}-\mathrm{D} F_{\mathrm{c}}$ electron density map of the heteroduplex, contoured at $1.5 \sigma$, is rendered as a grey mesh. Structurally disordered thymine nucleobase for which no unambiguous density is present is in grey. (D) Zoomed-in view of the $\mathrm{rA-dG}$ base pair at position 19 and the unpaired rU-dG mismatch at position 20 in AAVS1 off-target \#5 complex. Arrows indicate conformational changes in the TS backbone relative to the on-target complex. 
A

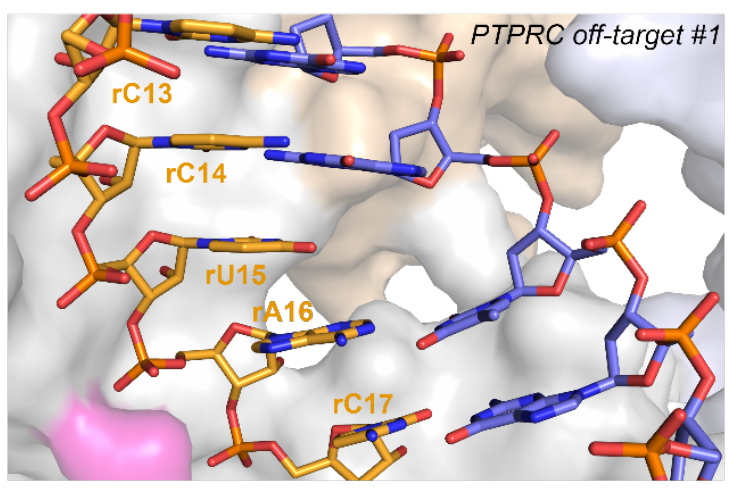

C

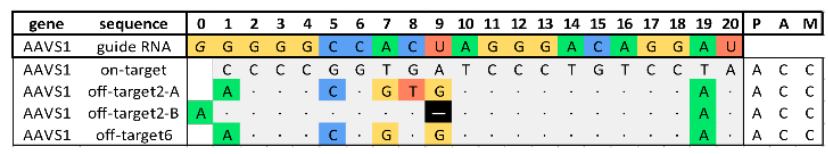

E

$\mathbf{F}$
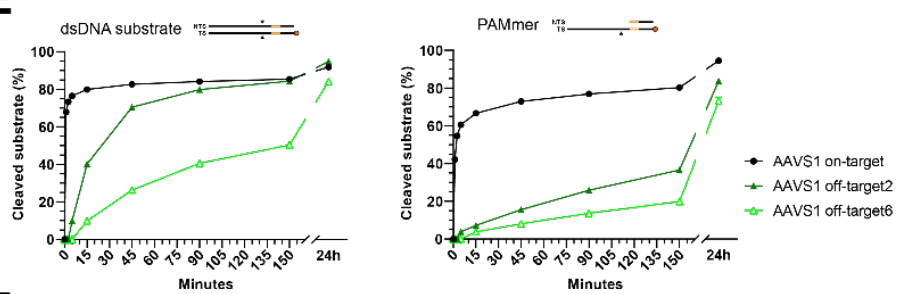

B

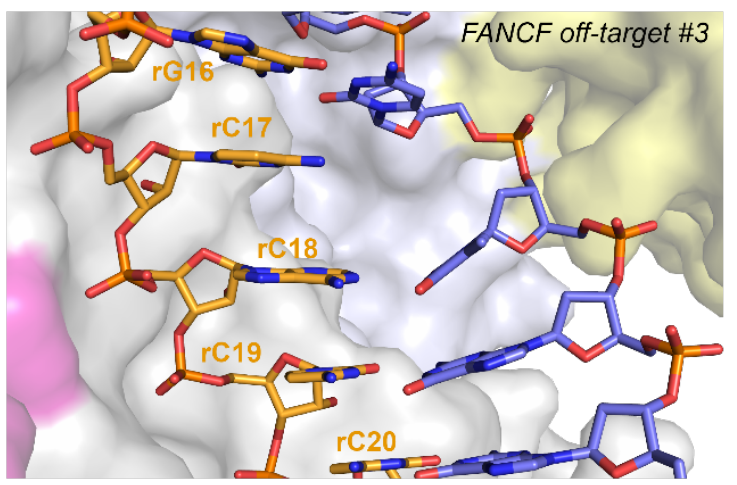

D

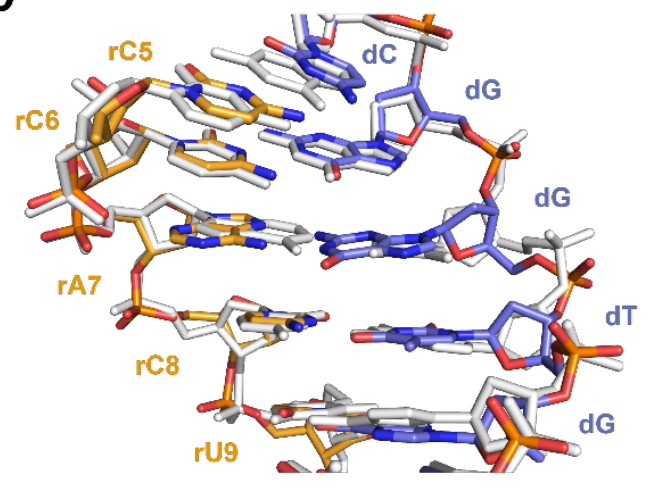

Figure 5

Figure 5. Off-targets with single-nucleotide deletions are accommodated by base skipping or multiple consecutive mismatches.

(A) Zoomed-in view of the base skip at duplex position 15 in the PTPRC-tgt2 off-target \#1 complex. (B) Zoomed-in view of the base skip at duplex position 17 in the FANCF off-target

714 \#3 complex. (C) Schematic depiction of alternative base pairing interactions in the AAVS1 off715 target \#2 complex. AAVS1 off-target \#6 substrate was designed based on the AAVS1 off-target $716 \# 2$, with the reversal of a single mismatch in the consecutive region back to the corresponding 717 canonical base pair. (D) Structural overlay of the AAVS1 off-target \#2 (coloured) and AAVS1 718 on-target (white) heteroduplexes. (E) Cleavage DNA kinetics of AAVS1 on-target, off-target 719 \#2 and off-target \#6 substrates. (F) Kinetic and thermodynamic parameters determined for 720 AAVS1 off-target \#2 and \#6 substrates. The apparent cleavage rate constants $\left(\mathrm{k}_{\mathrm{obs}}\right)$ were derived 
721 from a single-exponential function fitting of measured cleavage. Substrate binding $\left(\mathrm{k}_{\mathrm{on}}\right)$ and

722 dissociation ( $\mathrm{k}_{\mathrm{off}}$ ) constants were determined using SwitchSENSE assay.

723 
A

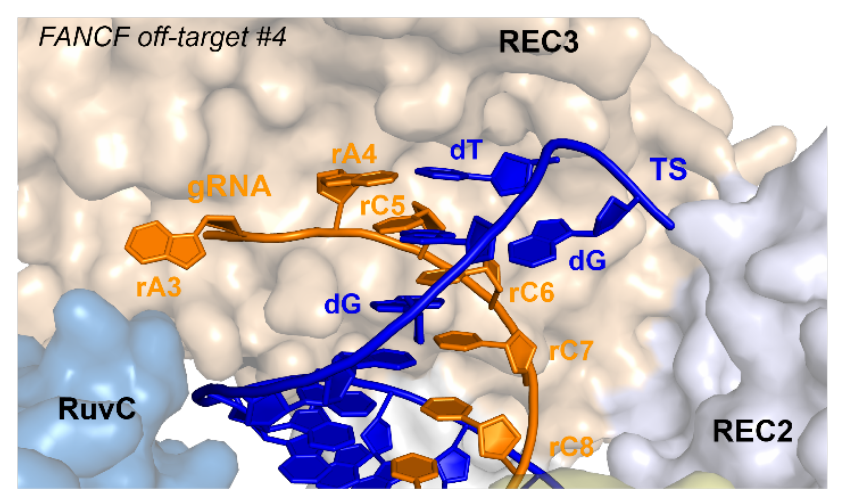

Figure 6

B

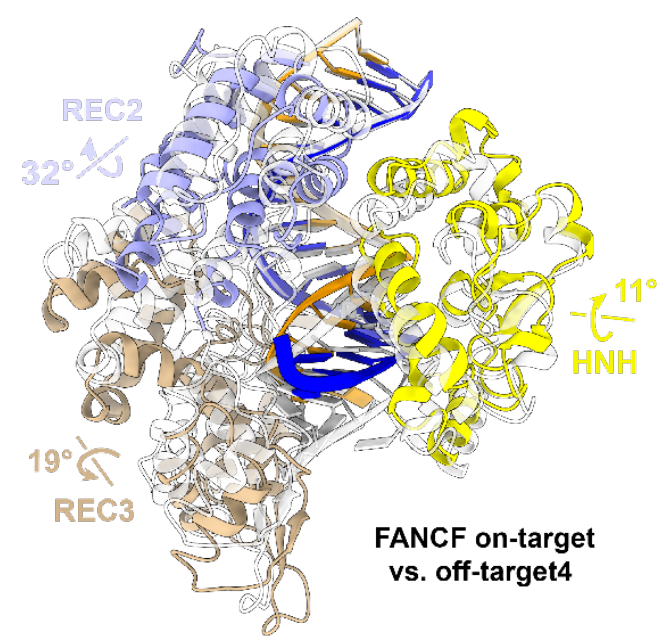

Figure 6. FANCF off-target \#4 exhibits conformational changes in the REC2/3 and HNH domains due to PAM-distal duplex unpairing.

727 (A) Close-up view of the unpairing of mismatched bases at the PAM-distal end of the FANCF 728 off-target \#4 heteroduplex. The last two nucleotides on each strand could not be modelled due 729 to structural disorder. (B) Overlay of the FANCF off-target \#4 and FANCF on-target complex 730 structures. The FANCF off-target \#4 complex is coloured according to the domain legend in 731 Figure 1A, FANCF on-target complex is shown in white. The REC1, RuvC, and PAM732 interaction domains have been omitted for clarity, as no structural differences are observed. 
A
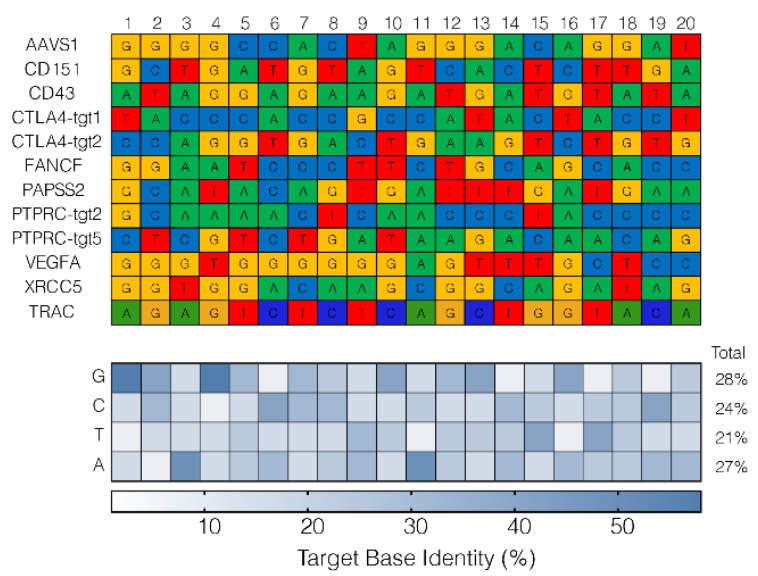

C

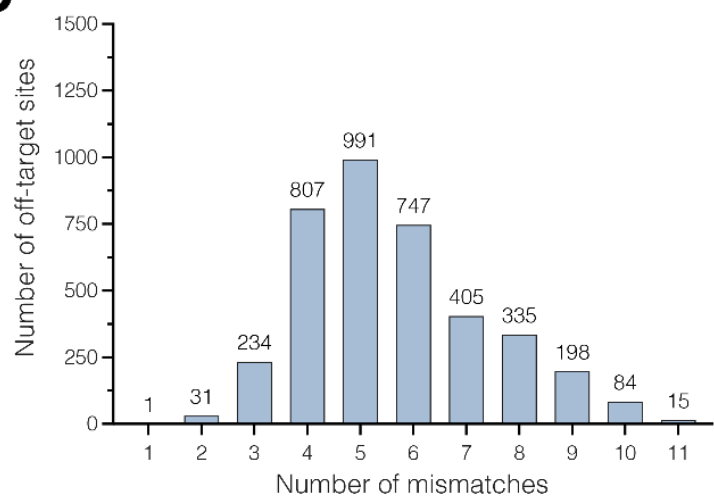

D

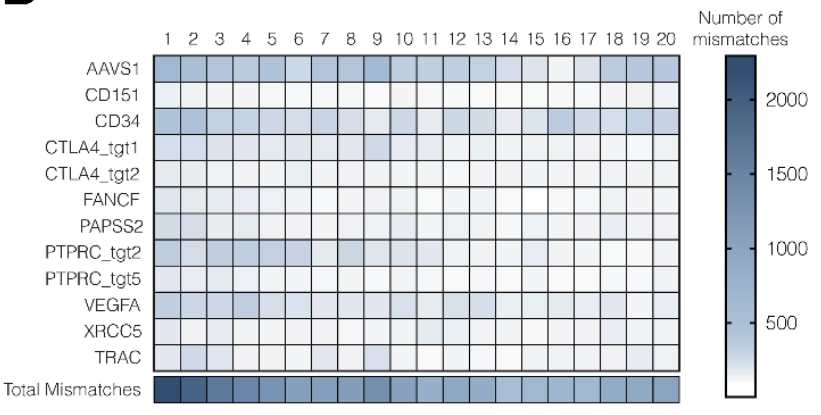

B

\begin{tabular}{|c|c|c|c|c|}
\hline \multicolumn{5}{|c|}{ SITE-Seq $\circledast$ assay recovered sites } \\
\hline & $4 \mathrm{nM}$ & $16 \mathrm{nM}$ & $64 \mathrm{nM}$ & $256 \mathrm{nM}$ \\
\hline AAVS1 & $\begin{array}{l}\text { onv/ } \\
19\end{array}$ & 55 & 269 & $\begin{array}{l}\text { orv } \\
1025\end{array}$ \\
\hline CD151 & \begin{tabular}{|l|}
$m v 1)$ \\
2
\end{tabular} & ${ }^{\operatorname{lom} v} 6$ & 28 & 79 \\
\hline & कात & का & & \\
\hline CD34 & 3 & 11 & 153 & 690 \\
\hline CTLA4-tgt 1 & 4 & 25 & 108 & 232 \\
\hline CTLA4-tgt2 & 3 & 7 & 26 & $\begin{array}{l}\text { Crv } \\
157\end{array}$ \\
\hline FANCF & 4 & 9 & 56 & 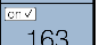 \\
\hline & (anv & & & \\
\hline PAPSS2 & 2 & 17 & 73 & 216 \\
\hline & (anv) & & $\sqrt{\text { Cenv }}$ & cavd \\
\hline PIPRC-tgt2 & 4 & $2 b$ & 146 & 350 \\
\hline PTPRC-tgt5 & 6 & 11 & 43 & 110 \\
\hline & ma & anve & & \\
\hline VEGFA & 4 & 29 & 141 & 433 \\
\hline XRCC5 & 0 & 3 & 36 & 146 \\
\hline TRAC & 11 & 50 & 147 & 247 \\
\hline Total sites & 62 & 248 & 1226 & 3848 \\
\hline Total & 253 & 1143 & 6448 & 21732 \\
\hline
\end{tabular}

$\mathbf{E}$

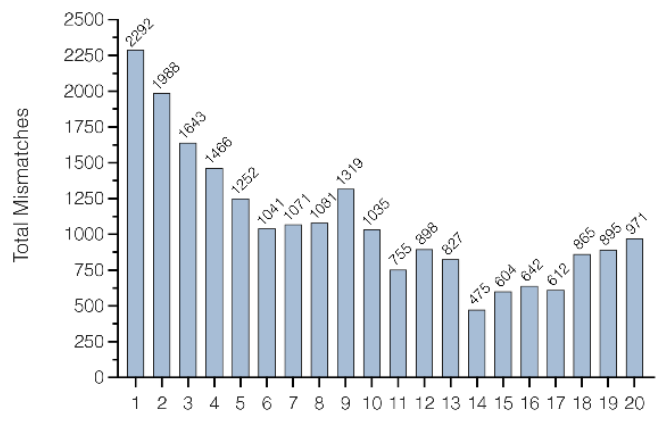

position in spacer

\section{Figure S1. Off-target profiling of selected genomic sites using SITE-Seq.}

735 (A) Selected genomic targets and the corresponding guide RNA sequences selected for the

736 SITE-Seq assay off-target profiling. Heatmap indicates frequency of nucleotide identity across

737 each position for the selected targets. (B) SITE-seq assay analysis for RNPs assembled with

738 indicated crRNAs. The numbers of detected off-target sites are shown as a function of RNP

739 concentration. Checked boxes indicate recovery of the on-target site. $\mathrm{n}=3$ replicates per sample.

740 (C) Number of off-target sites recovered by the SITE-Seq assay are shown as a function of the 
741 number of mismatches between the guide RNA and the off-target sequence. (D) Frequency of

742 nucleotide mismatches at each guide RNA-off-target DNA heteroduplex position for all off-

743 target sites identified in (B). (E) Number of total identified mismatches per heteroduplex

744 position.

745 
A dsDNA substrate ${ }_{\mathrm{Ts}}^{\mathrm{Ns}}=$
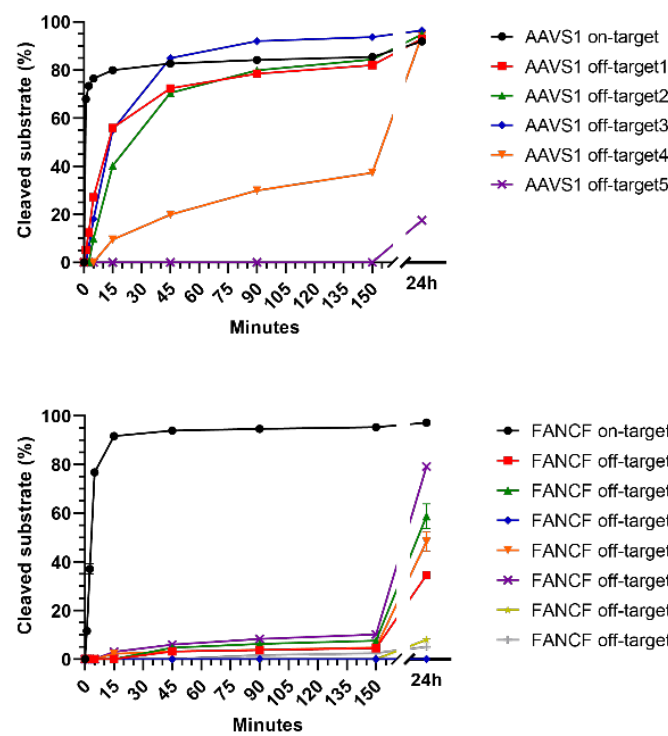

- FANCF on-target

- FANCF off-target1

- FANCF off-target2

$\rightarrow$ FANCF off-target3

- FANCF off-target4

* FANCF off-target5

- FANCF off-target6

- FANCF off-target?

Minutes
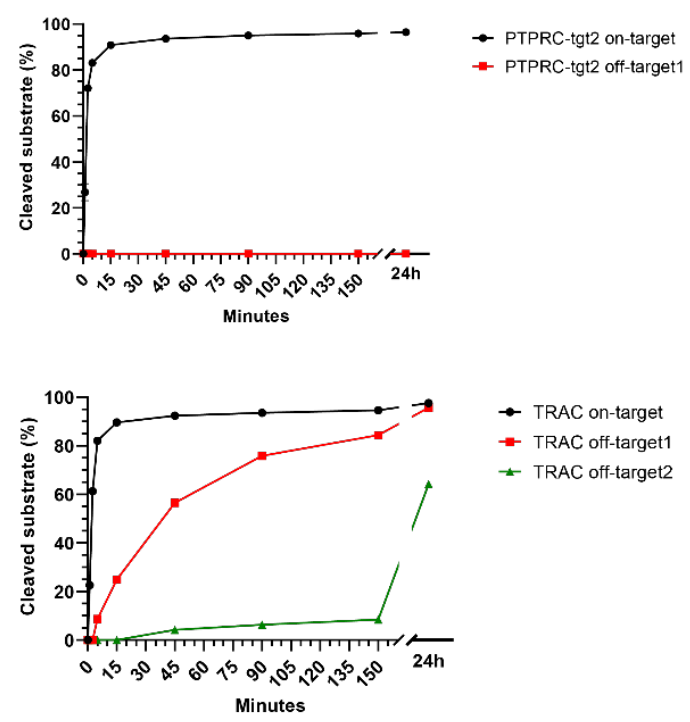

C

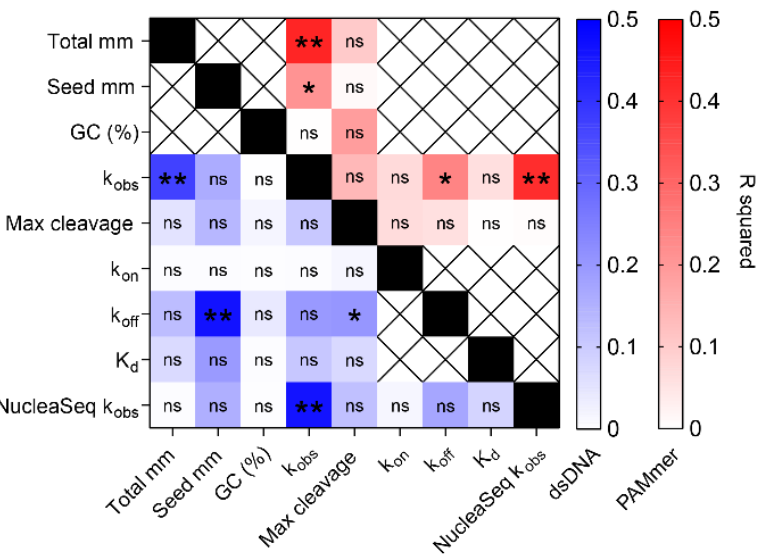

B
Figure S2
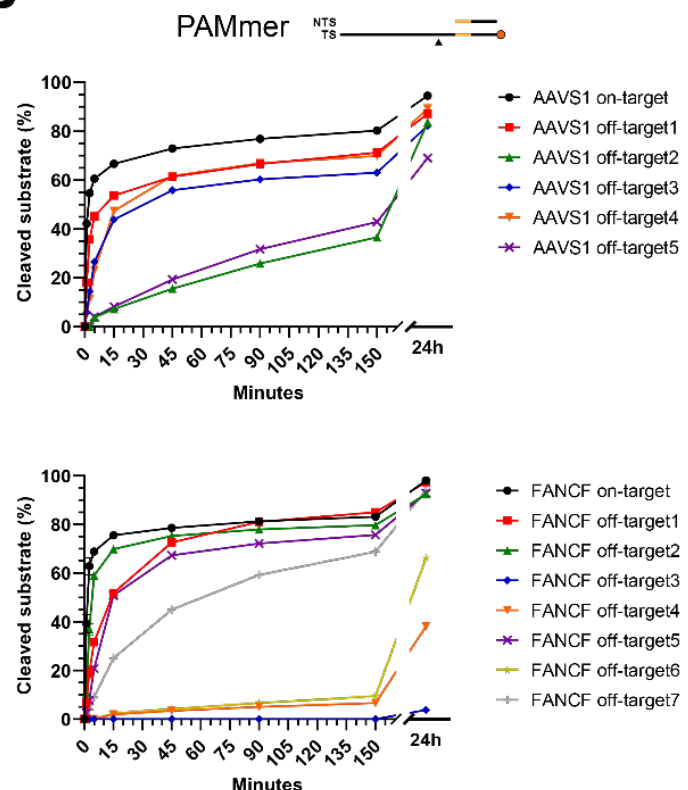

- FANCF on-target

- FANCF off-target1

- FANCF off-target2

- FANCF off-target3

- FANCF off-target4

* FANCF off-target5

- FANCF off-target6

+ FANCF off-target7
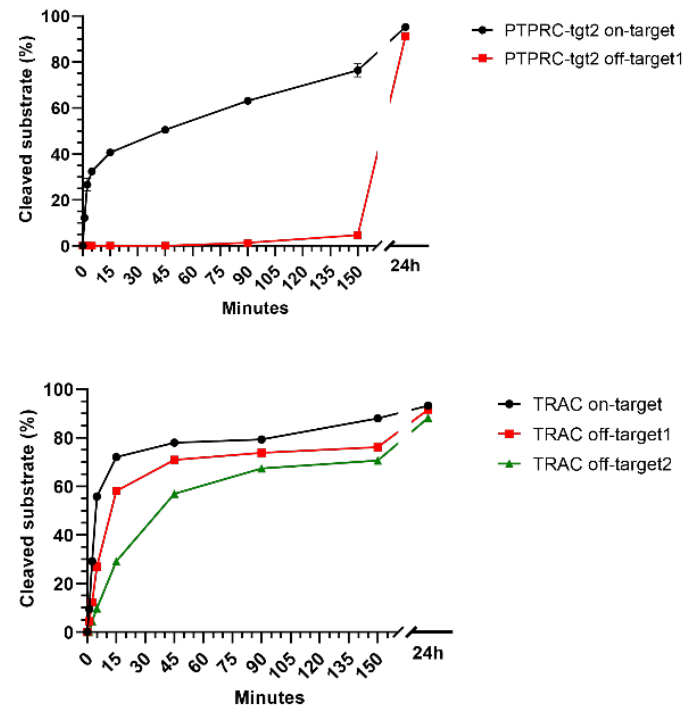

D

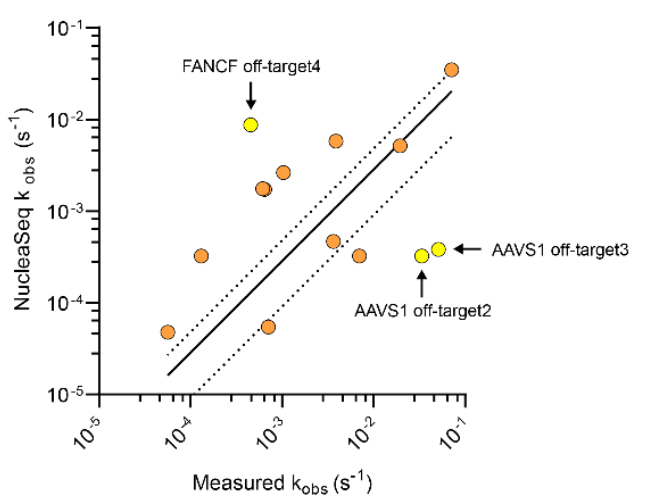

747 Figure S2. in vitro cleavage of selected Cas9 off-target substrates. 
748 (A) In vitro clevage kinetics of fully double stranded on- and off-target DNA substrates for each

749 guide RNA used in the study. Black triangles in the substrate schematic (top) indicate position

750 of cleavage sites. Each data point represents a mean of four independent replicates. Error bars

751 represent standard deviation for each time point. (B) In vitro clevage kinetics of partially single

752 stranded (PAMmer) on- and off-target substrates. (C) Heatmap representation of mutual

753 correlations between measured kinetic and thermodymamic parameters including cleavage

$754\left(\mathrm{k}_{\text {obs }}\right)$, substrate DNA binding $\left(\mathrm{k}_{\text {on }}\right)$, substrate dissociation $\left(\mathrm{k}_{\text {off }}\right)$ rate constants, equilibrium

755 dissociation constant $\left(\mathrm{K}_{\mathrm{d}}\right)$ with numbers of nucleotide mismatches in the off- target sites (total

756 and within seed), the GC content of the spacer $(\% \mathrm{GC})$ and cleavage rate predicted using the

757 NucleaSeq algorithm (NucleaSeq $\mathrm{k}_{\mathrm{obs}}$ ). The values were calculated across all off-targets for

758 both dsDNA (lower left half, in blue), and partially single stranded (PAMmer) substrates (upper

759 right half, in red). ns, no significant correlation. (D) Correlation between measured and

760 NucleaSeq-predicted $\mathrm{k}_{\mathrm{obs}}$ rate constants. Off-target sites with significant deviations are

761 highlighted in yellow. 


\section{A}
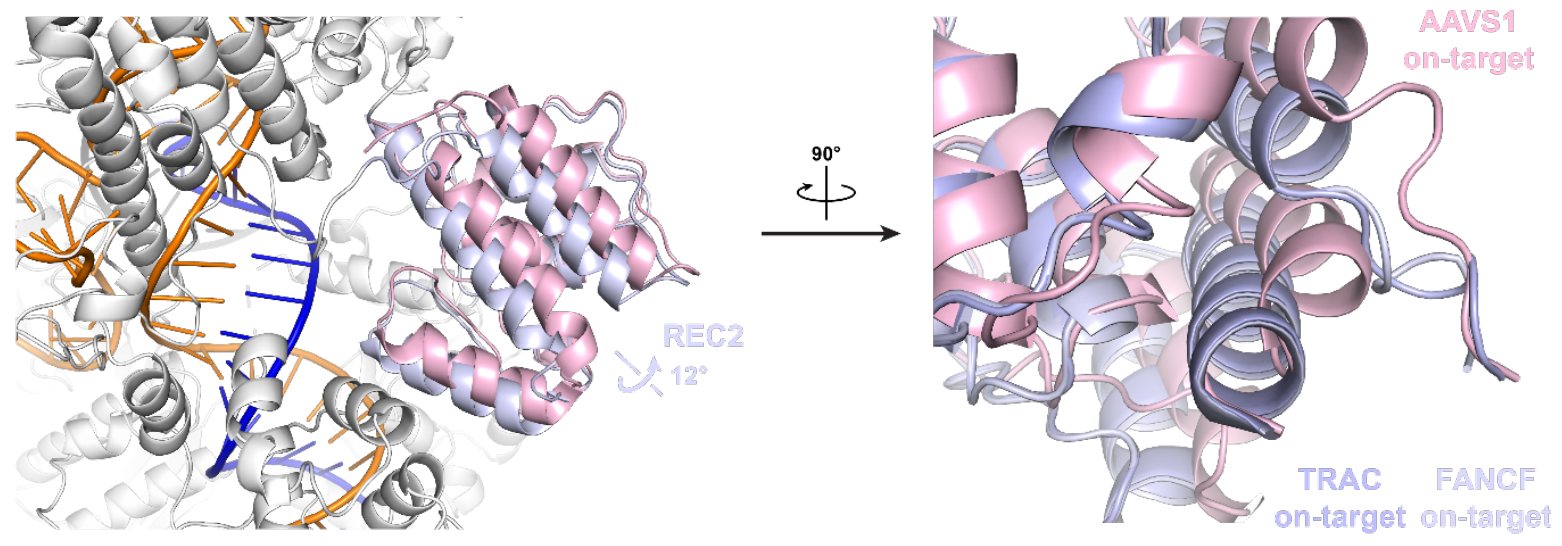

B

\section{Figure S3. Alternative REC2 conformation in AAVS1 on-target.}

(A) Overlay of REC2 domain conformations in the AAVS1 (pink), FANCF (purple) and TRAC

(light blue) on-target complexes (B) Close-up view of helix REC2 helix spanning Cas9 residues 
A

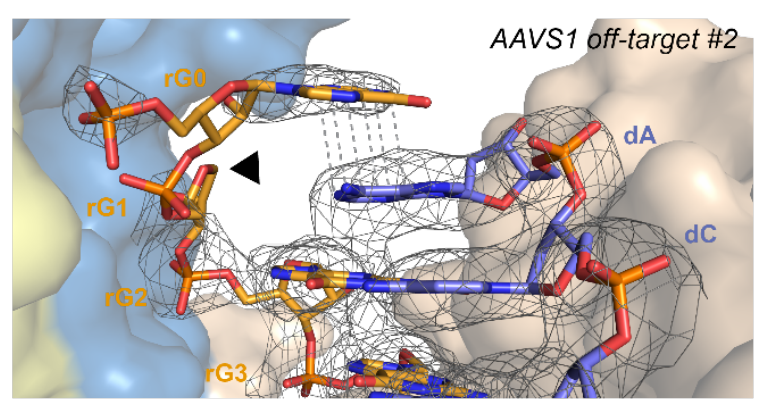

C

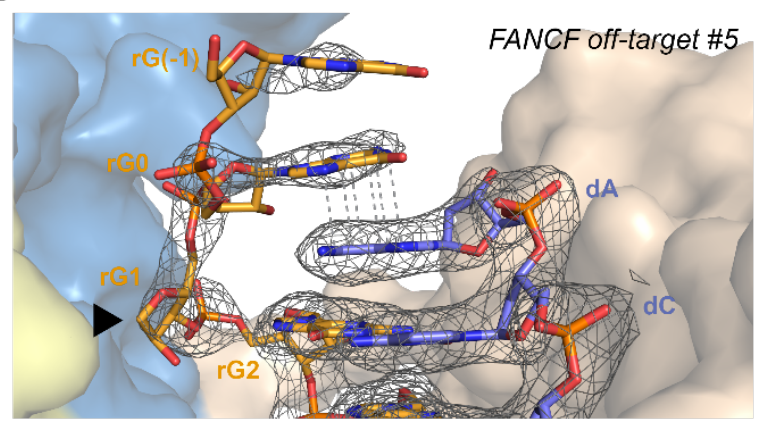

\section{Figure S4}

B

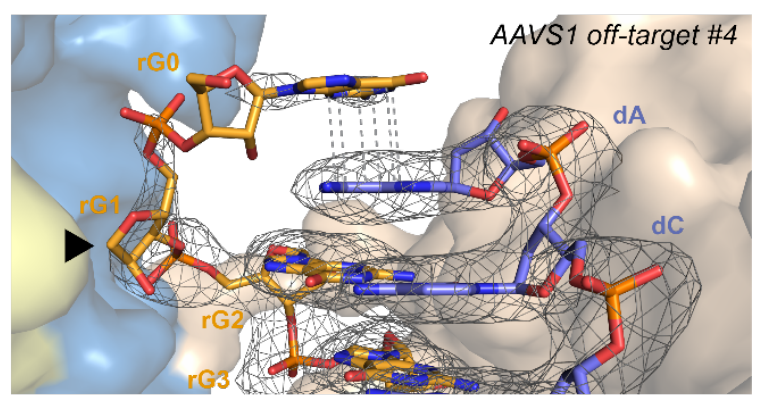

D

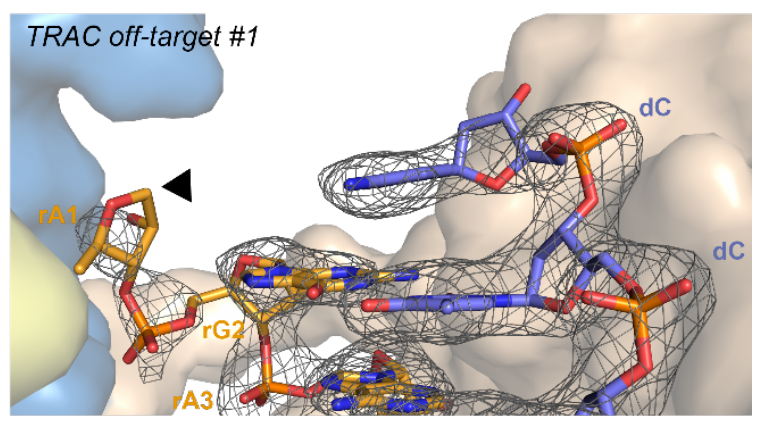

Figure S4. PAM-distal mismatches result in unpairing and disordering of guide RNA for (A) and $1.0 \sigma$ for (B)-(D).

\section{nucleobase in position 1.}

Close-up views of the PAM-distal end of the guide RNA-TS heteroduplex in (A) AAVS1 offtarget \#2, (B) AAVS1 off-target \#4, (C) FANCF off-target \#5 and (D) TRAC off-target \#1 complexes. Arrowheads indicate nucleotides with disordered bases. Refined $2 \mathrm{~m} F_{\mathrm{o}}-\mathrm{D} F_{\mathrm{c}}$ electron density maps of the heteroduplexes are rendered as a grey mesh and contoured at $1.2 \sigma$ 

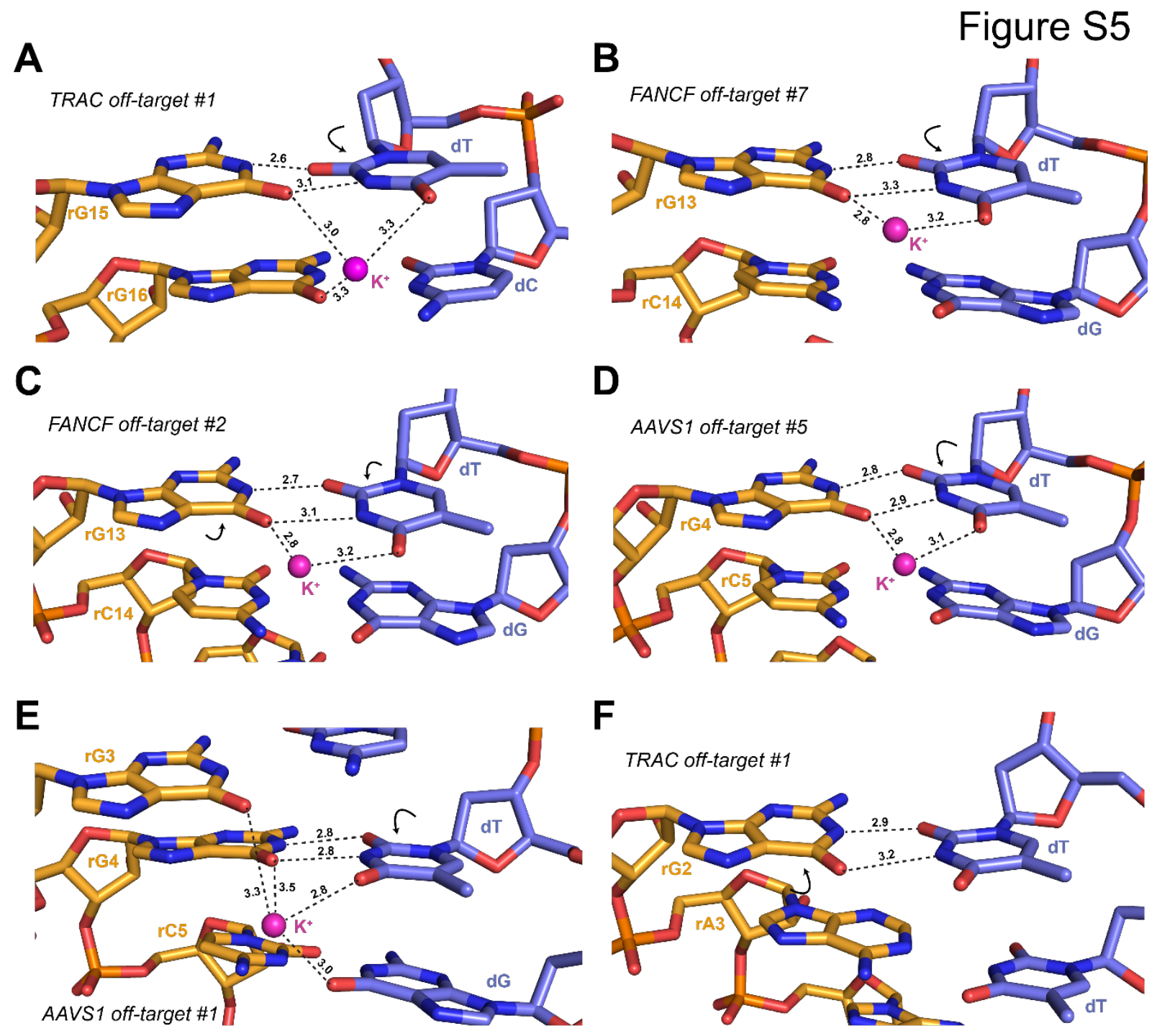

\section{G}

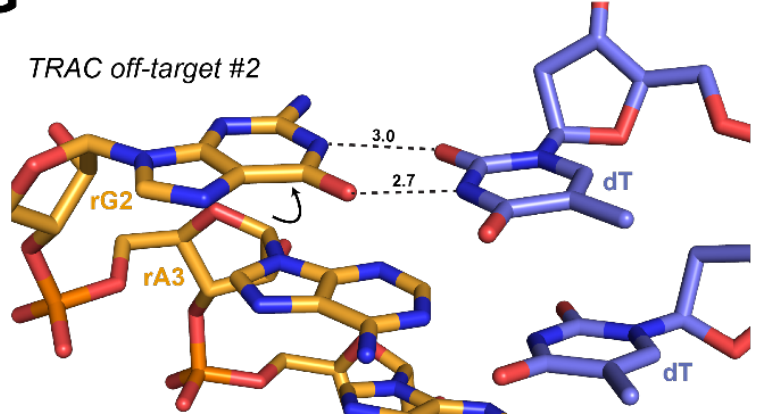

781 Figure S5. Wobble base pairing of rG-dT mismatches.

782 Close-up views of rG-dT mismatches at (A) heteroduplex position 15 in the TRAC off-target

783 \#1 complex, (B) position 13 in FANCF off-target \#7 complex, (C) position 13 in FANCF off-

784 target \#2 complex, (D) position 4 in AAVS1 off-target \#5 complex, (E) position 4 in AAVS1 off-

785 target \#1 complex, (F) position 2 in TRAC off-target \#1 complex and (G) position 2 in TRAC 
786 off-target \#2 complex. Arrows indicate conformational changes relative to the corresponding

787 on-target complex structures. Monovalent ions, modeled as $\mathrm{K}^{+}$, are depicted as purple spheres.

788 In (A)-(E), the dT base is displaced into the major groove and forms a canonical wobble base

789 pair with the $\mathrm{rG}$ base. In $(\mathrm{F})-(\mathrm{G})$, the the $\mathrm{rG}$ base instead shifts towards the minor groove to

790 facilitate wobble pairing.

791

792

793

794 
A

\section{C}
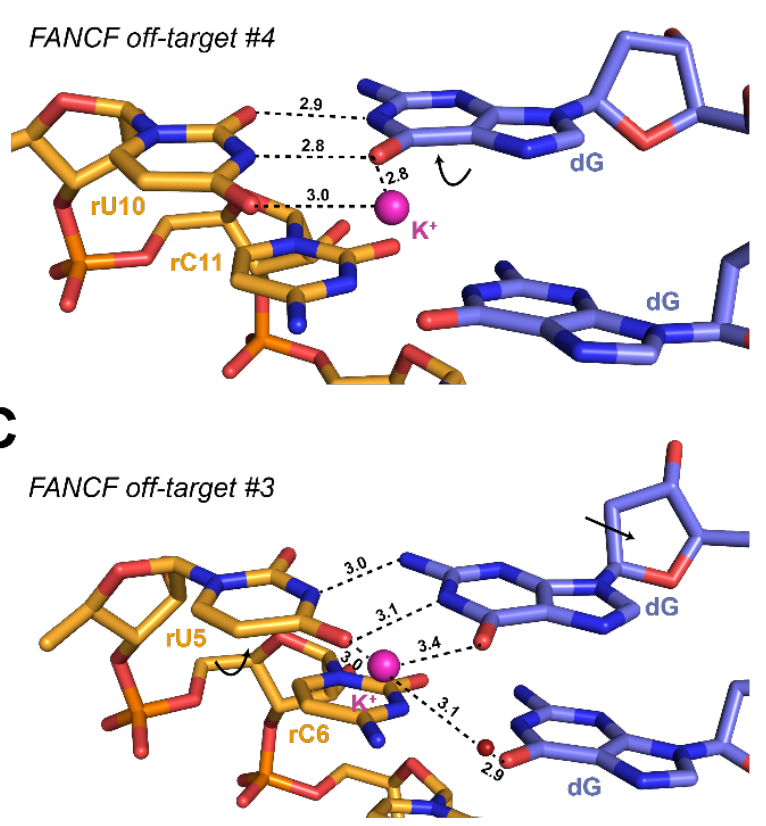

$\mathbf{E}$

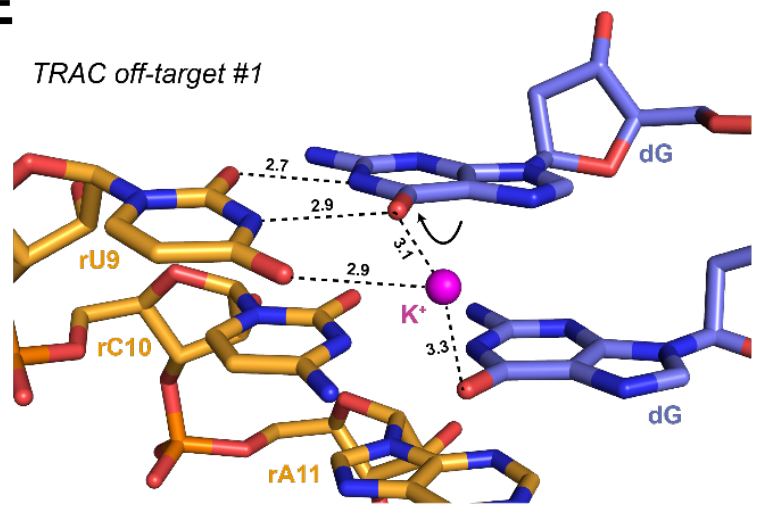

G

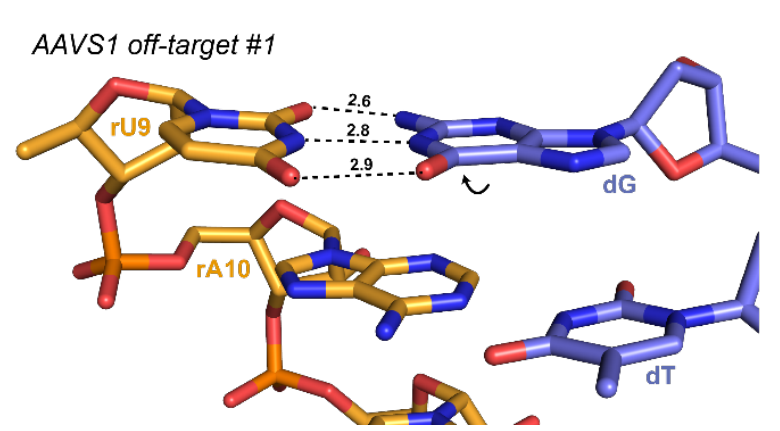

B

Figure S6

D
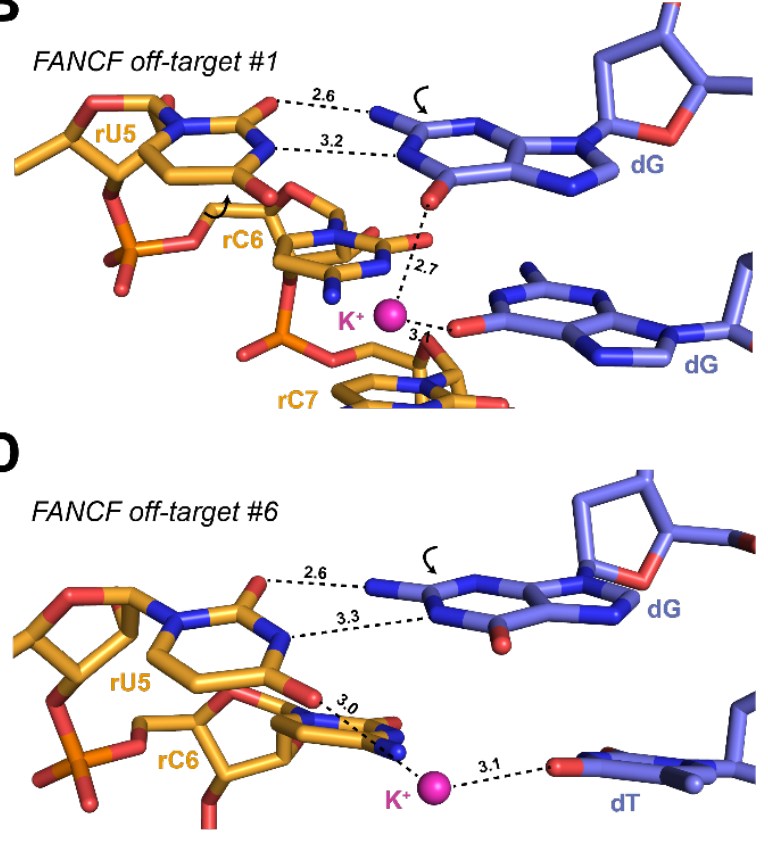

$\mathbf{F}$

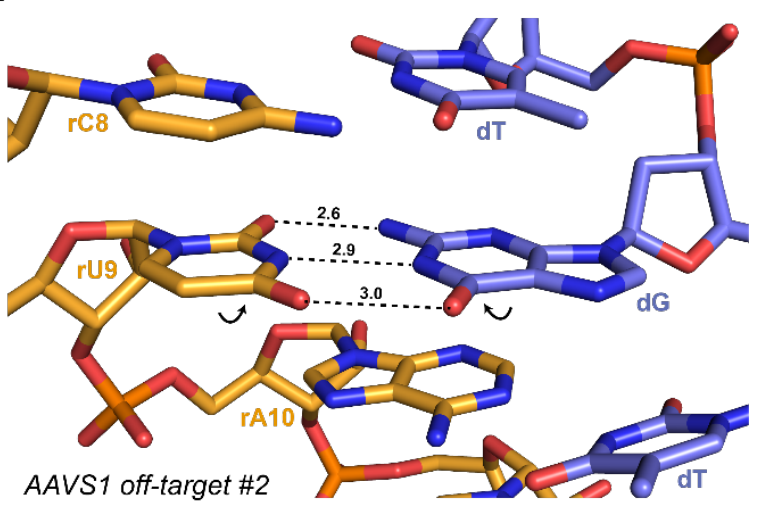

H<smiles></smiles>

keto-enol-Uracil amino-dGuanine

Figure S6. rU-dG wobble base pairs adopt duplex position-dependent conformations.

797 Close-up views of of rU-dG mispairs at (A) heteroduplex position 10 in FANCF off-target \#4

798 complex, (B) position 5 in FANCF off-target \#1 complex, (C) position 5 in FANCF off-target \#3 complex, (D) position 5 in FANCF off-target \#6 complex, (E) TRAC off-target \#1 complex, 
800 and (F) AAVS1 off-target \#2, and (G) AAVS1 off-target \#1 complex. Arrows indicate 801 conformational changes relative to the corresponding on-target complex structures. Bound 802 potassium ions are depicted as purple spheres. In (A), rU-dG wobble base pairing is achieved 803 by minor groove displacement of the guanine base. In (B)-(D), the rU-dG mispairs adopt 804 atypical conformations. In (E), the guanine base is shifted into the minor groove to form a 805 wobble base pair, whereas at the identical heteroduplex position in (F) and (G), the rU-dG base 806 pairs do not engage in wobble pairing, instead adopting alternative tautomeric forms. (H) 807 Schematic depicting hydrogen bonding interactions between $\mathrm{rU}$ and $\mathrm{dG}$ bases in $(\mathrm{F})$ and $(\mathrm{G})$. 808 
A

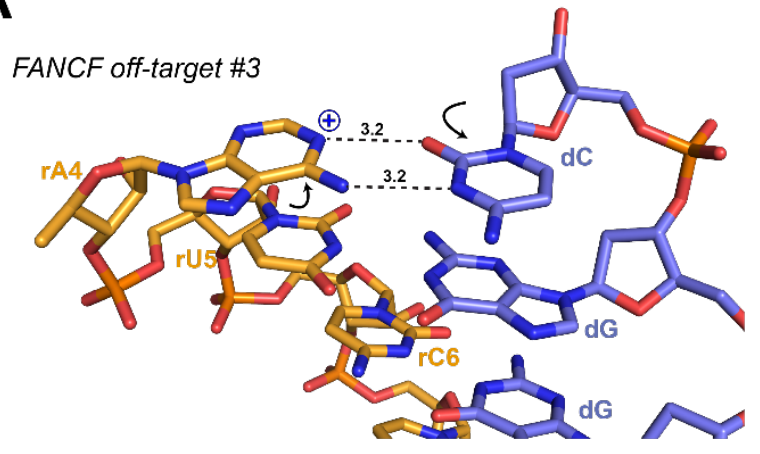

C

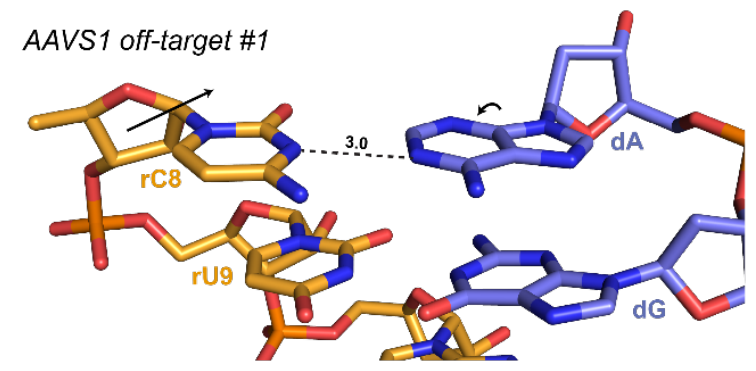

$\mathbf{E}$

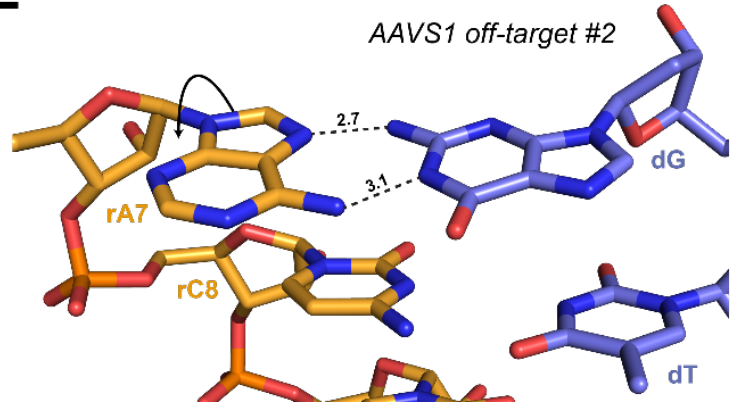

B

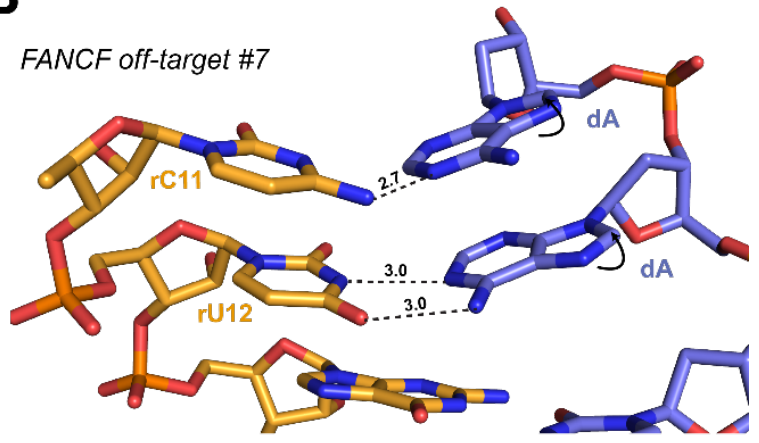

D

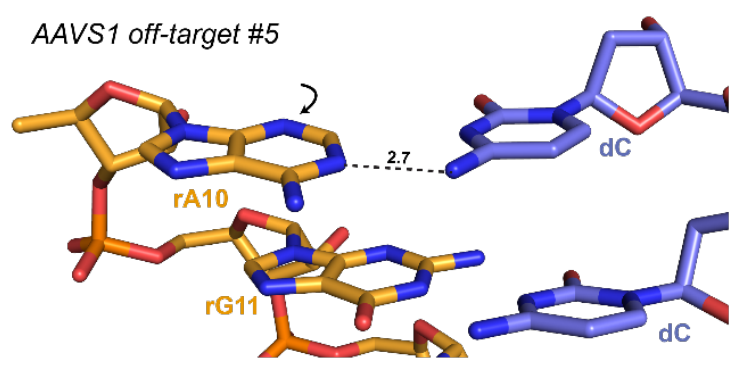

809

Figure S7. Additional non-canonical base pairs within Cas9 off-target complexes.

817 view of Hoogsteen-edge rA-dG base pair at position 7 in AAVS1 off-target \#2 complex. Arrows

818 indicate conformational changes relative to the corresponding on-target complexes. 
Figure S8

A

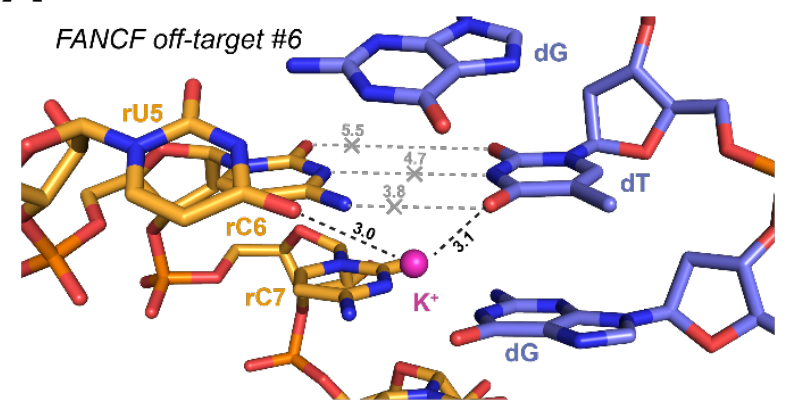

C

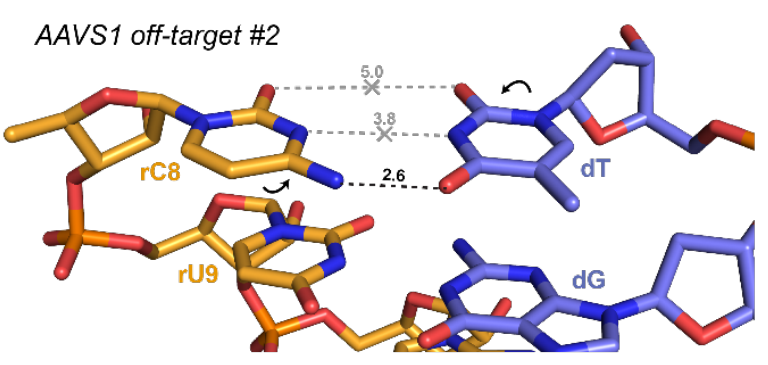

$\mathbf{E}$

PTPRC-tgt2 off-target \#1

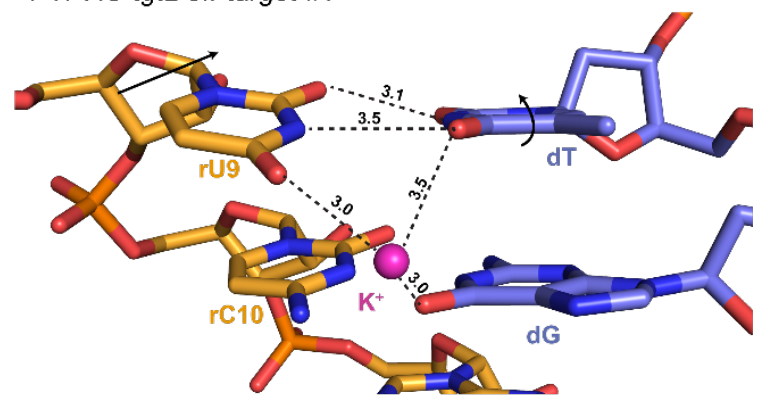

G

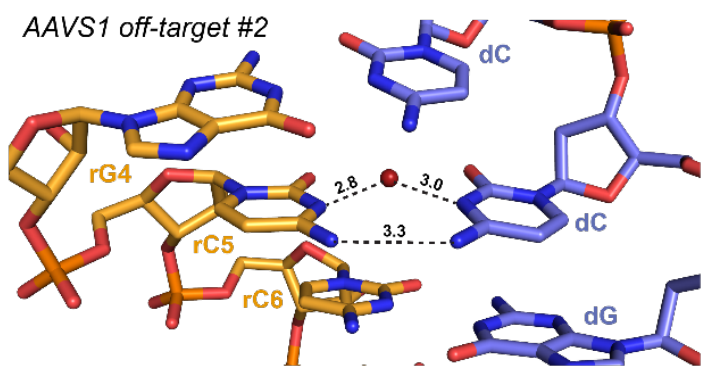

B

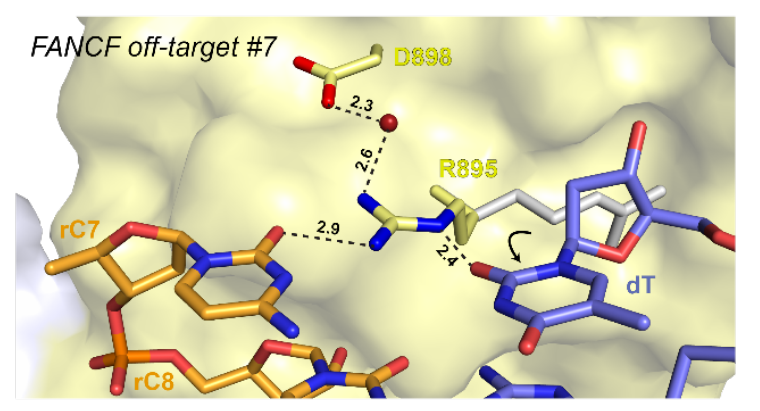

D

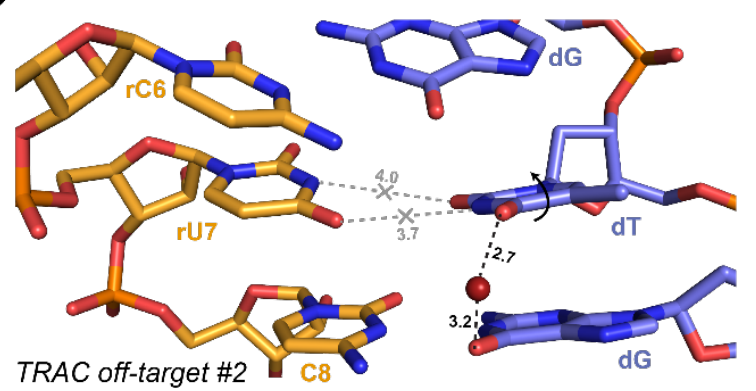

F

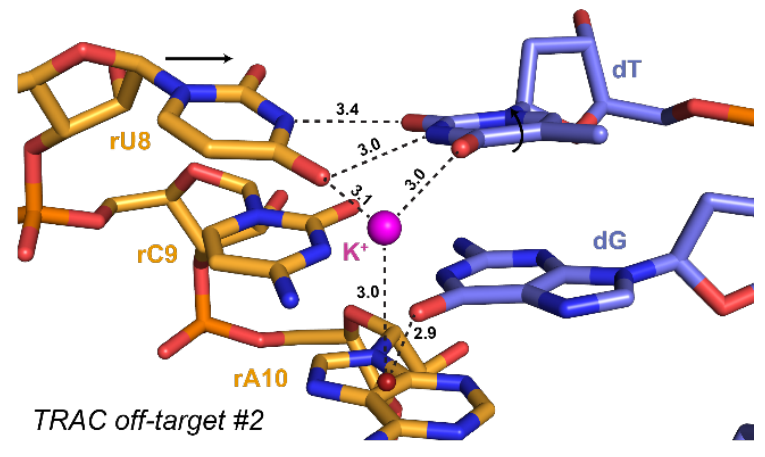

H

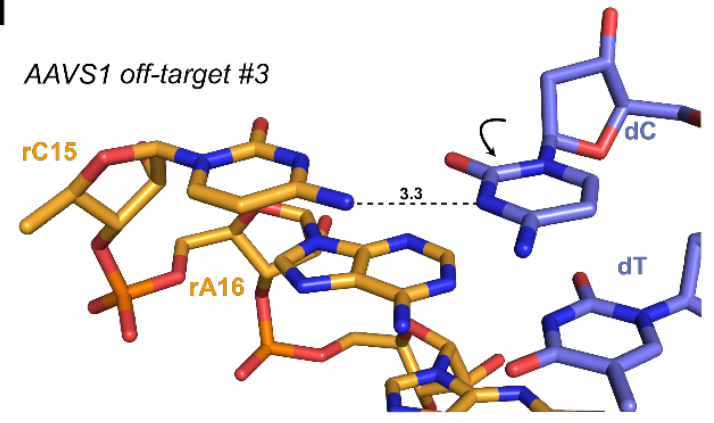

820 Figure S8. Preservation of base stacking in pyrimidine-pyrimidine off-target mismatches.

821 (A) Close-up view of rC-dT mispair at position 6 in FANCF off-target \#6 complex. (B) Close-

822 up view of rC-dT mispair at position 7 in FANCF off-target \#7 complex, bridged by Arg895.

823 The arginine sidechain in the corresponding on-target complex is shown in white. (C) Close-

824 up view of rC-dT base pairing at position 8 of AAVS1 off-target \#2. (D) Close-up view of rU- 
825 dT mispair at position 7 in TRAC off-target \#2 complex. (E) Close-up view of rU-dT pairing at 826 position 9 in $P T P R C$-tgt2 off-target \#1 complex, facilitated by base propeller twisting. (F)

827 Close-up view of rU-dT pairing at position 8 in TRAC off-target \#2 complex, enabled by 828 backbone shift of the RNA strand. (G) Close-up view of partially paired rC-dC mismatch at 829 position 5 in AAVS1 off-target \#2 complex, bridged by a water molecule. (H) Close-up view of 830 rC-dC mispair at position 15 in AAVS1 off-target \#3 complex.

831 


\section{A}

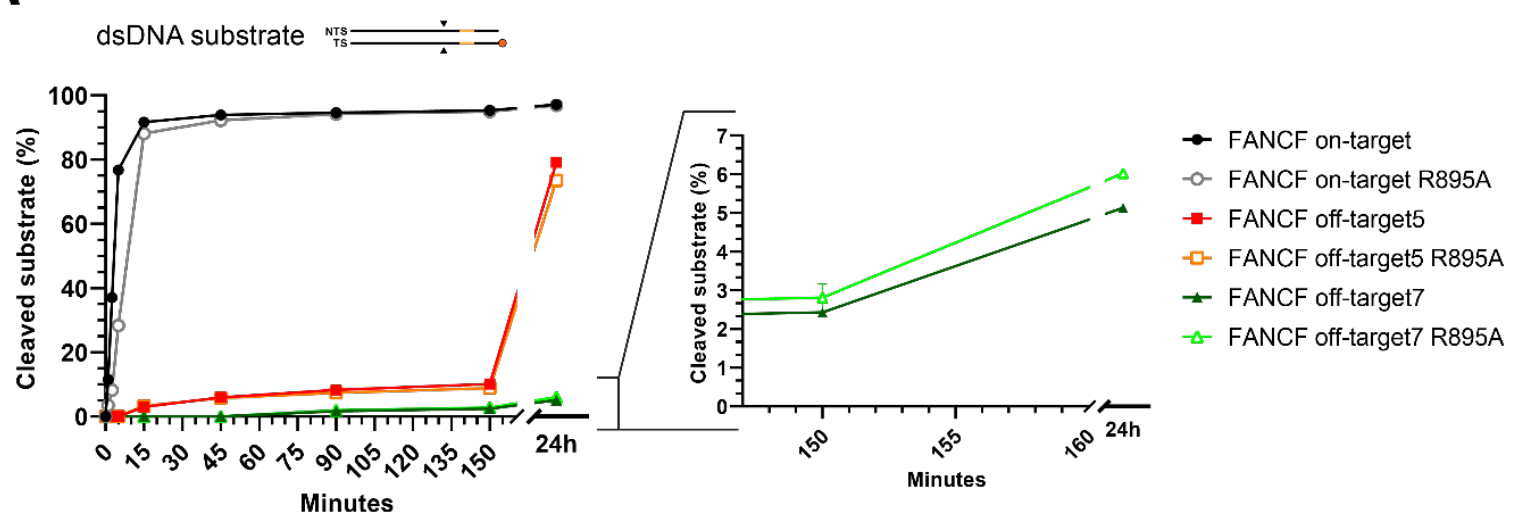

B

\begin{tabular}{|c|c|c|c|c|c|c|c|c|c|}
\hline Gene & Target & Cas9 & $\begin{array}{l}\text { (\%) } 24 \mathrm{~h} \\
\text { cleavage }\end{array}$ & $k_{\text {obs }}\left(\min ^{-1}\right)$ & $k_{\text {on }}\left(M^{-1} \cdot s^{-1}\right)$ & $k_{\text {off }}\left(s^{-1}\right)$ & $\mathrm{K}_{\mathrm{d}}(\mathrm{pM})$ & $\begin{array}{l}\text { 24h cleavage } \\
\text { PAMmer (\%) }\end{array}$ & $\begin{array}{c}\mathbf{k}_{\text {obs }}\left(\min ^{-1}\right) \\
\text { PAMmer }\end{array}$ \\
\hline FANCF & on-target & $W T$ & 97.5 & 0.2383 & $4.02 E+06$ & $6.77 \mathrm{E}-05$ & 16.9 & 98.3 & 0.5654 \\
\hline FANCF & on-target & R895A & 97.1 & 0.0945 & $4.87 \mathrm{E}+06$ & 7.03E-05 & 14.5 & 97.9 & 0.4214 \\
\hline FANCF & off-target7 & WT & 5.2 & 0.0036 & $5.23 E+06$ & 2.90E-03 & 555.0 & 94.5 & 0.0134 \\
\hline FANCF & off-target7 & R895A & 6.1 & 0.0036 & $5.06 E+06$ & 2.36E-03 & 466.0 & 96.5 & 0.0142 \\
\hline
\end{tabular}

\section{Figure S9. Cas9 R895A mutation of Cas9 has no significant impact on FANCF off-target}

\section{\#7 cleavage or binding.}

836 (A) Kinetic analysis of FANCF on- and off-target substrate DNA cleavage by wild-type and

837 R895A Cas9 proteins. (B) Kinetic and thermodynamic parameters of FANCF on- and off-target

838 substrate DNA cleavage by wild-type and R895A Cas9. Cleavage rate constants $\left(\mathrm{k}_{\text {obs }}\right)$ were

839 derived from single-exponential function fitting of plots shown in (A). Substrate binding and

840 dissociation rate constants $\left(\mathrm{k}_{\text {on }}\right.$ and $\left.\mathrm{k}_{\text {off }}\right)$ and the equilibrium dissociation constant $\left(\mathrm{K}_{\mathrm{d}}\right)$ were

841 determined using a DNA nanolever (switchSENSE) binding assay. 


\section{a}

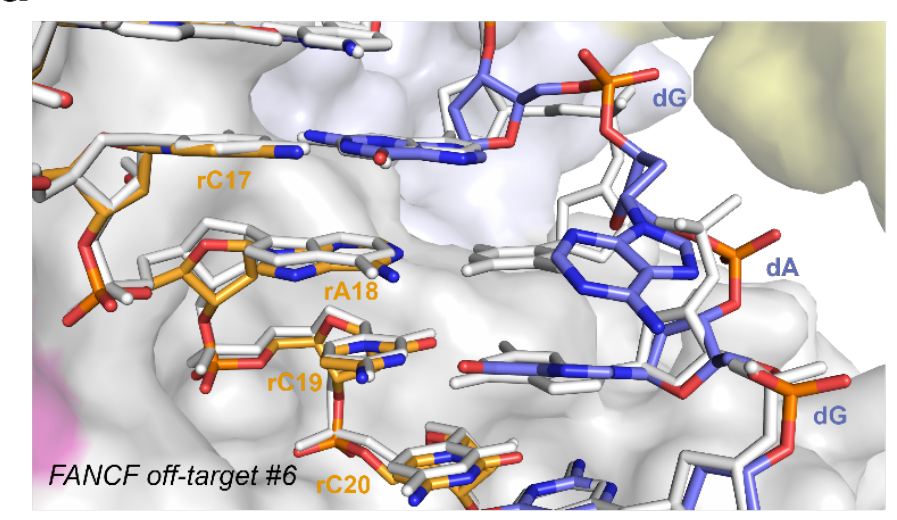

b

\begin{tabular}{|c|c|c|c|c|c|c|c|c|c|c|c|c|c|c|c|c|c|c|c|c|}
\hline & 1 & 2 & 3 & 4 & 5 & 6 & 7 & 8 & 9 & 10 & 11 & 12 & 13 & 14 & 15 & 16 & 17 & 18 & 19 & 20 \\
\hline Total Mismatches & 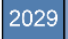 & 1771 & 1453 & 1295 & 1100 & 882 & 888 & 924 & 1171 & 885 & 632 & 769 & 738 & 409 & 506 & 524 & 484 & 708 & 775 & 801 \\
\hline dA-rA mismatches & 139 & 36 & 322 & 74 & 66 & 97 & 72 & 39 & 51 & 85 & 49 & 15 & 7 & 57 & 2 & 24 & 16 & 89 & 155 & 148 \\
\hline \%dA-rA mismatches & $10.8 \%$ & $9.5 \%$ & $7.8 \%$ & $6.9 \%$ & $5.9 \%$ & $4.7 \%$ & $4.7 \%$ & $4.9 \%$ & $6.3 \%$ & $4.7 \%$ & $3.4 \%$ & $4.1 \%$ & $3.9 \%$ & $2.2 \%$ & $2.7 \%$ & $2.8 \%$ & $2.6 \%$ & $3.8 \%$ & $4.1 \%$ & $4.3 \%$ \\
\hline
\end{tabular}

\section{Figure S10. Tolerance of adenine-adenine mismatches within the heteroduplex.}

845 (A) Close-up view of rA-dA mismatch at position 18 in FANCF off-target \#6 complex, overlaid

846 with the FANCF on-target structure (white). (B) Number of rA-dA off-target mismatches per

847 heteroduplex position recovered in the SITE-Seq assay for all analysed genomic targets.

848 Percentages indicate frequency of rA-dA mismatches recovered in the particular position as a

849 fraction of total number of rA-dA mismatches. 
A

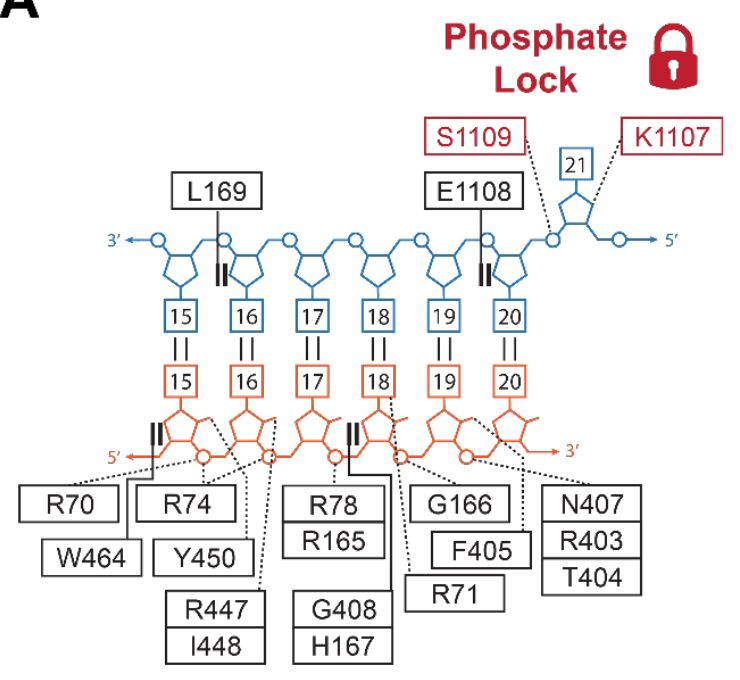

\section{C}

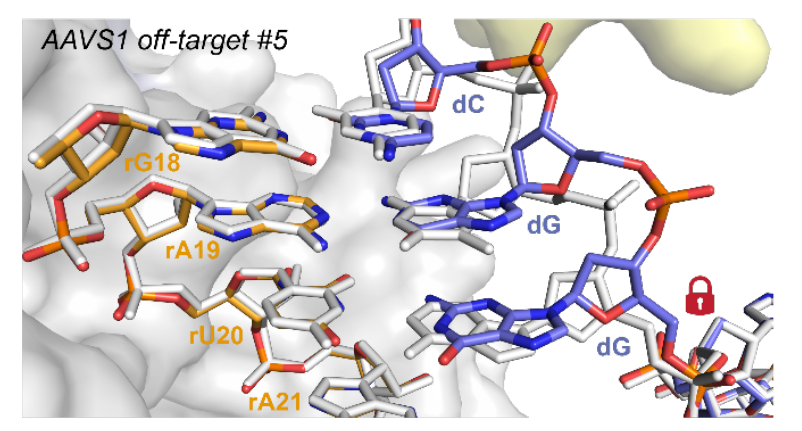

Figure S11

B

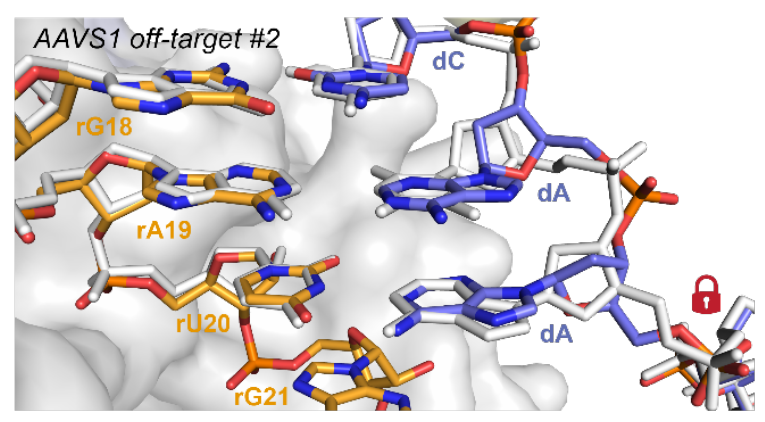

Figure S11. Lack of protein contacts with the target DNA strand in the seed region allows

for large phosphate backbone distortions.

854 (A) Schematic overview of Cas9 interactions within the PAM-proximal seed region of the guide

855 RNA-TS DNA heteroduplex. (B) Close-up view of the seed region in AAVS1 off-target \#2 856 complex, overlaid with the AAVS1 on-target heteroduplex (white), showing structural

857 distortion of the TS due to rA-dA mispair at seed position 19. (C) Close-up view of the seed 858 region in AAVS1 off-target \#5 complex, overlaid with the AAVS1 on-target heteroduplex 859 (white), showing structural distortion due to $\mathrm{rA}-\mathrm{dG}$ and $\mathrm{rU}-\mathrm{dG}$ mismatches at positions 19 and 86020 , respectively. Red lock icon indicates position of the phosphate lock residue in (B) and (C). 
A

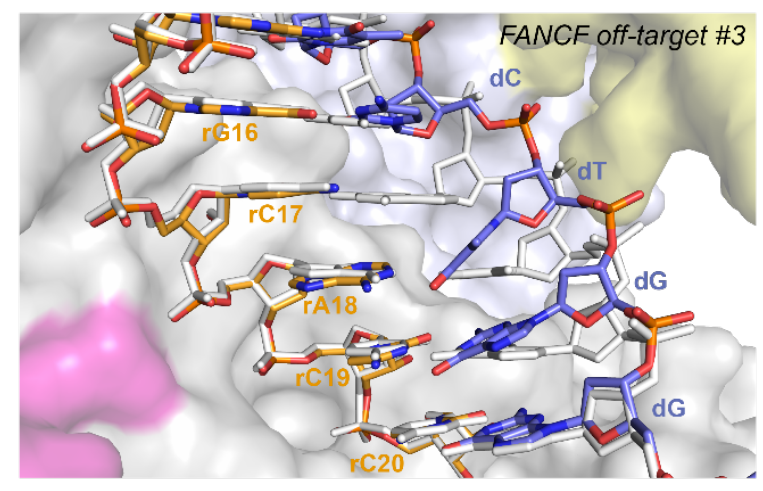

\section{C}

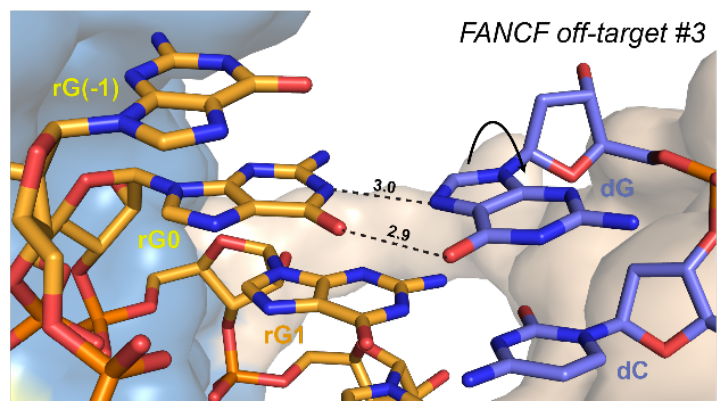

B

\section{Figure S12}

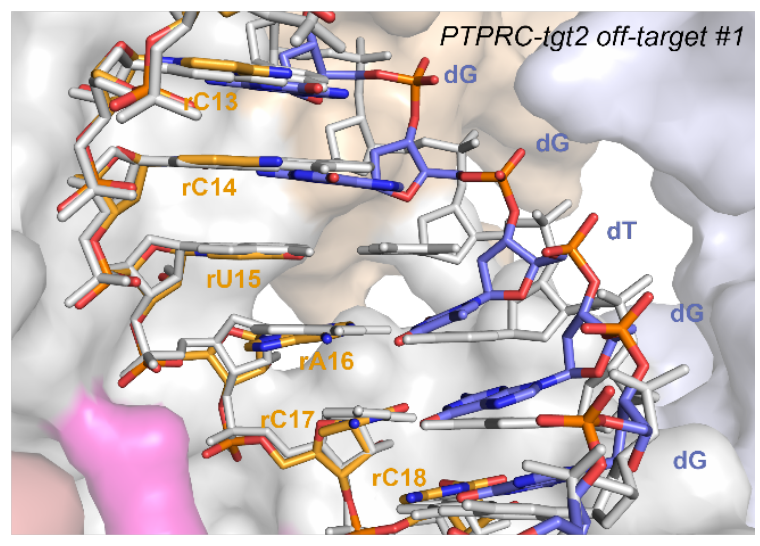

D

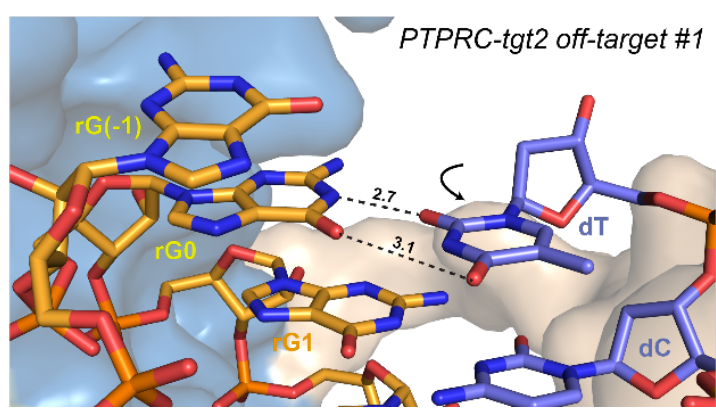

863 Figure S12. Recognition of off-target sites containing deletions in the seed region.

864 (A) Close-up view of base skipping within the seed region of the guide RNA-off-target DNA heteroduplex in FANCF off-target \#3 complex, overlaid with the on-target heteroduplex

(white). (B) Close-up view of base skipping within the seed region of the guide RNA-off-target

DNA heteroduplex in PTPRC-tgt2 off-target \#1 complex, overlaid with FANCF on-target heteroduplex (white). (C) Close-up view of non-canonical base pairs at the 5'-terminus of the guide RNA in FANCF off-target \#3 complex involving guanosine nucleotides introduced during in vitro transcription of the guide RNA. (D) Close-up view of non-canonical base pairs at the 5'-terminus of the guide RNA in PTPRC-tgt2 off-target \#1 complex involving guanosine nucleotides introduced during in vitro transcription of the guide RNA. 


\section{Figure S13}

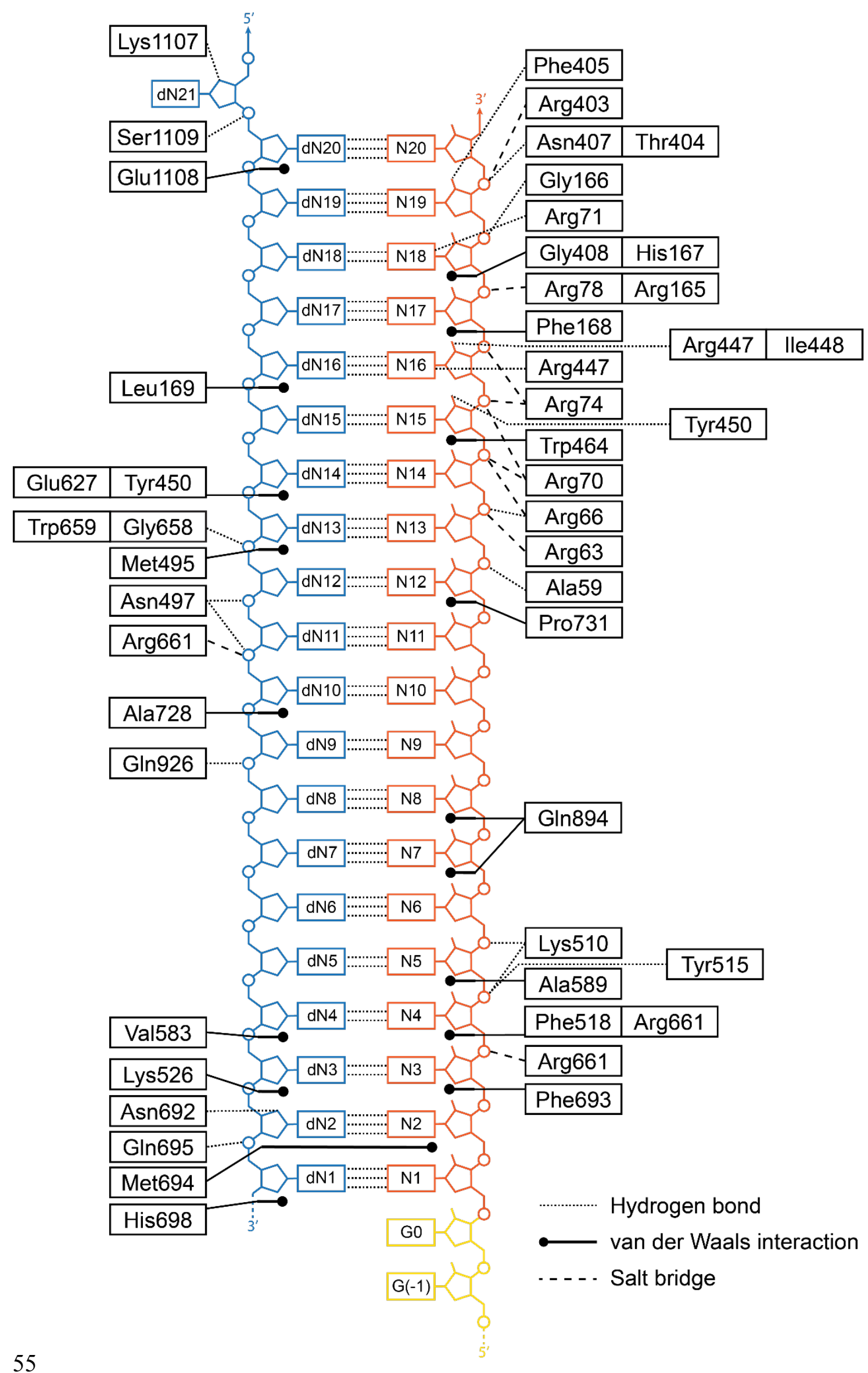


875 Figure S13. Cas9-nucleic acid interactions in on-target complexes.

876 Schematic diagram depicting Cas9 residues interacting with the guide RNA-target DNA

877 heteroduplex. Dotted lines represent hydrogen bonding interactions; dashed lines represent salt

878 bridges; solid lines represent stacking/hydrophobic interactions. Target strand is coloured in

879 blue, guide RNA in orange. Phosphates are represented by circles, ribose moieties by

880 pentagons, and nucleobases by rectangles.

881 


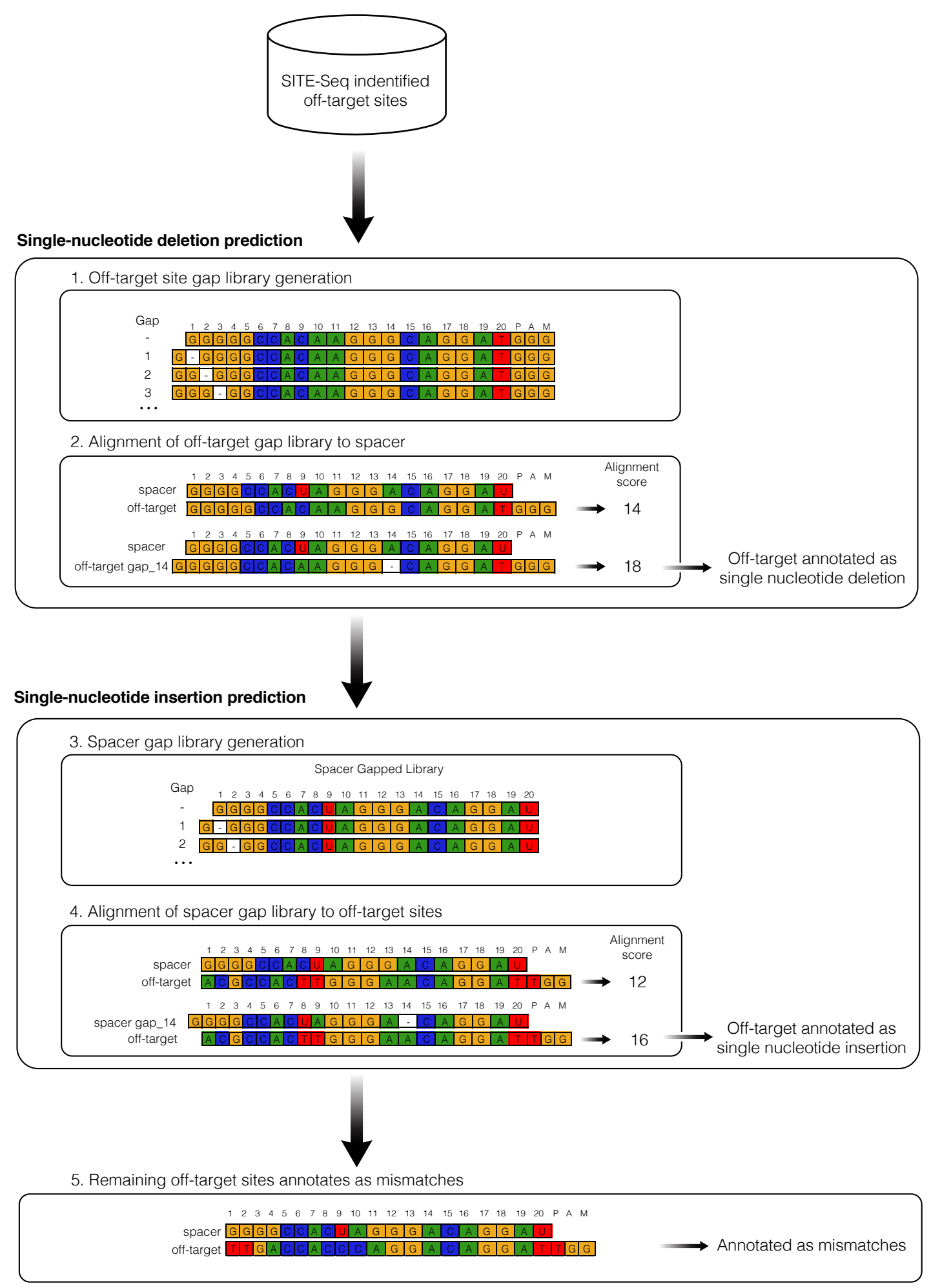

883 Figure S14. Schematic representation of mismatch, insertion, and deletion classification

884 algorithm for the SITE-Seq assay analysis of off-target sites. 
885 Schematic represents unrestricted classification algorithm of off-target sites with putative

886 insertions and deletions. In the final restricted pipeline, the positioning is limited to 887 heteroduplex positions 6-20 for insertions and positions 10-20 for deletions.

888 


\section{A}

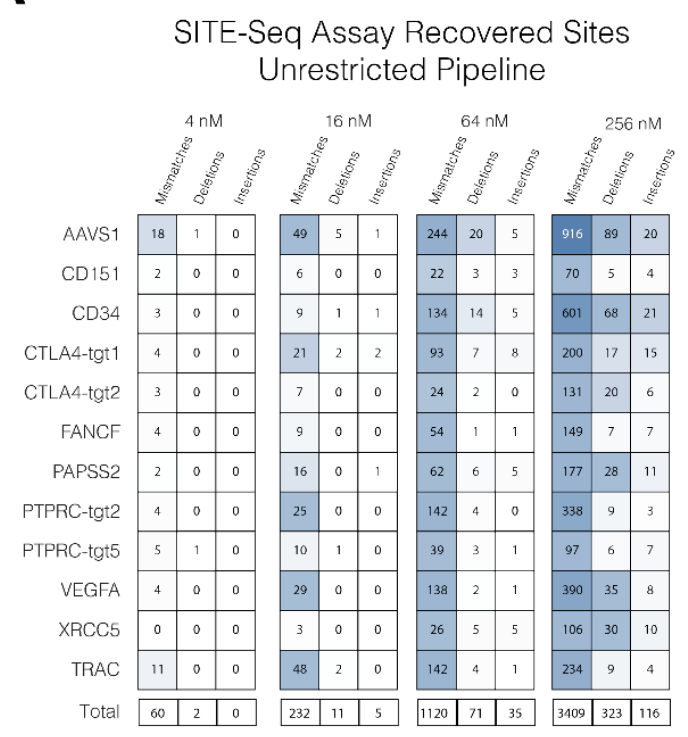

B

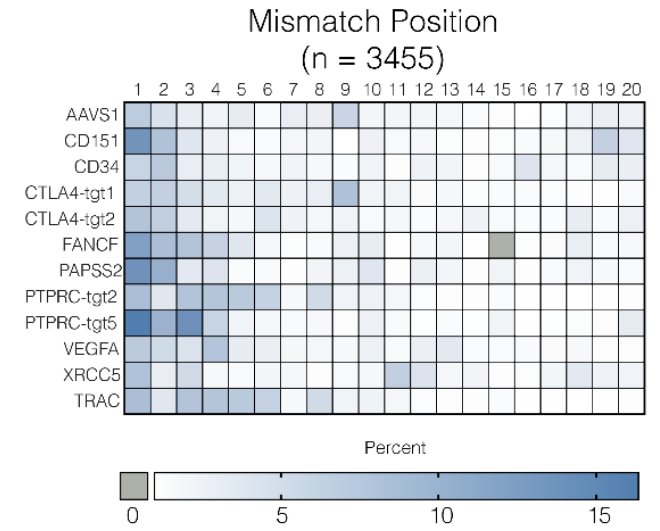

D Single-nucleotide Deletion Frequency $(n=277)$

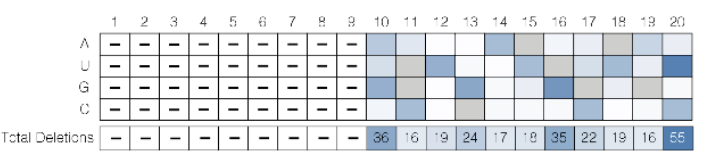

\section{Figure S15}

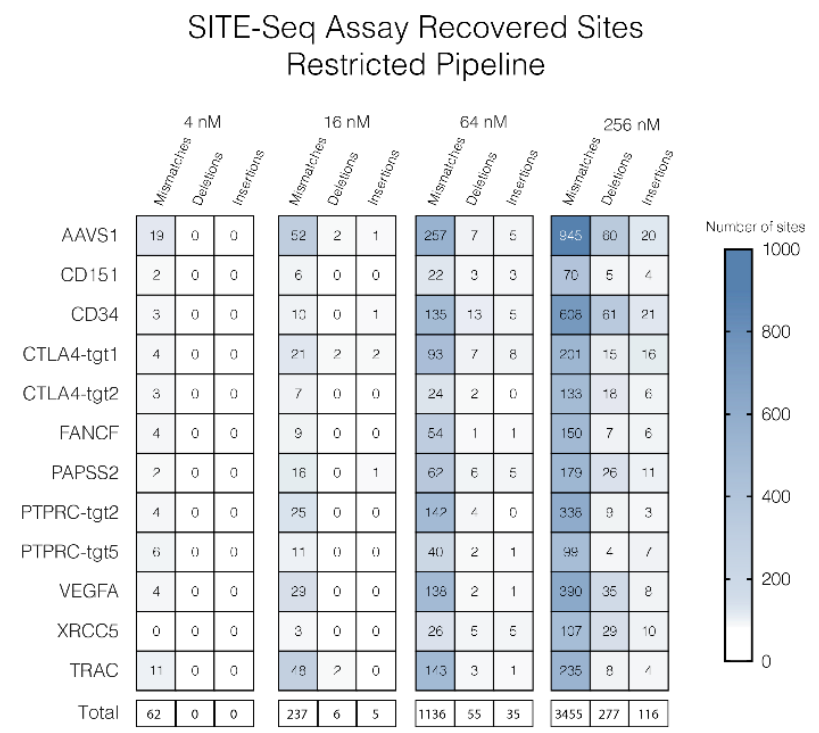

C

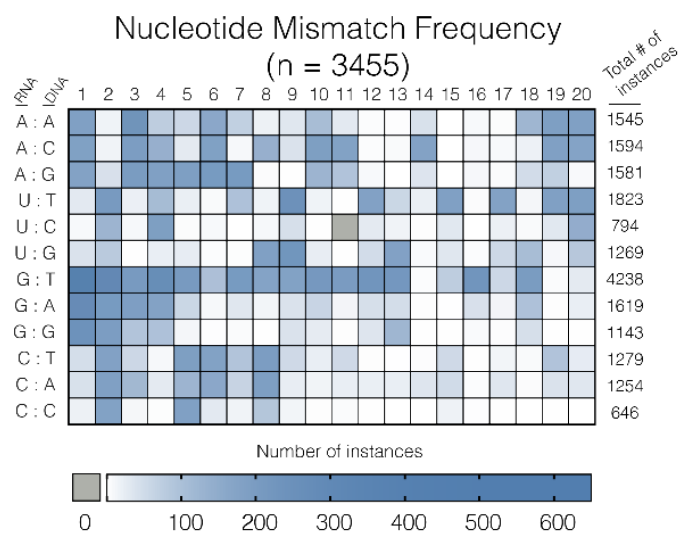

$\mathbf{E}$

Single-nucleotide Insertion Frequency $(n=116)$

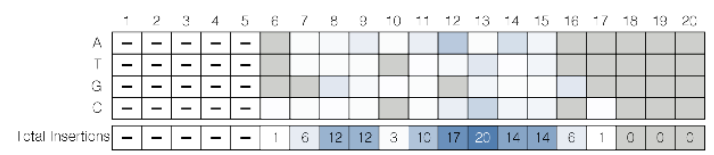

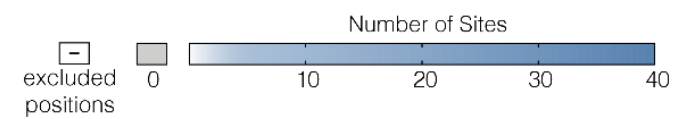

890 Figure S15. Positional restriction of nucleotide insertions/deletions during of SITE-Seq

891 assay off-target profiling.

892 (A) Number of recovered off-target sites per genomic target as a function of RNP concentration

893 classified as containing either only mismatches, single-nucleotide deletions, or single-

894 nucleotide insertions. Left panel corresponds to classification using algorithm with no 
895 positional restriction. Right panel corresponds to classification using algorithm restricting 896 deletions to positions 10-20 and insertions to 6-20 only. (B) Frequency of positional mismatch 897 occurrence per genomic target for mismatched off-targets with the positionally restricted

898 algorithm. (C) Frequency of nucleotide mismatches within the heteroduplex for all off-target 899 sites when classified with a positionally restricted pipeline ( $\mathrm{n}=3445$ sites for both (B) and (C)). 900 (D) Frequency of single-nucleotide deletions occurring within positions 10-20 of the 901 heteroduplex for all off-target sites when analysed with a positionally restricted pipeline. 902 ( $\mathrm{n}=277$ sites). (E) Frequency of single-nucleotide insertions occurring within positions 6-20 of 903 the heteroduplex for all off-target sites when analysed with a positionally restricted pipeline. $904 \quad(n=116$ sites $)$. 


\section{A}

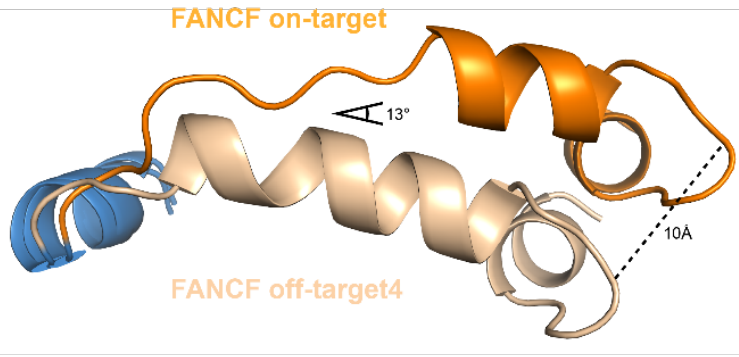

B

C
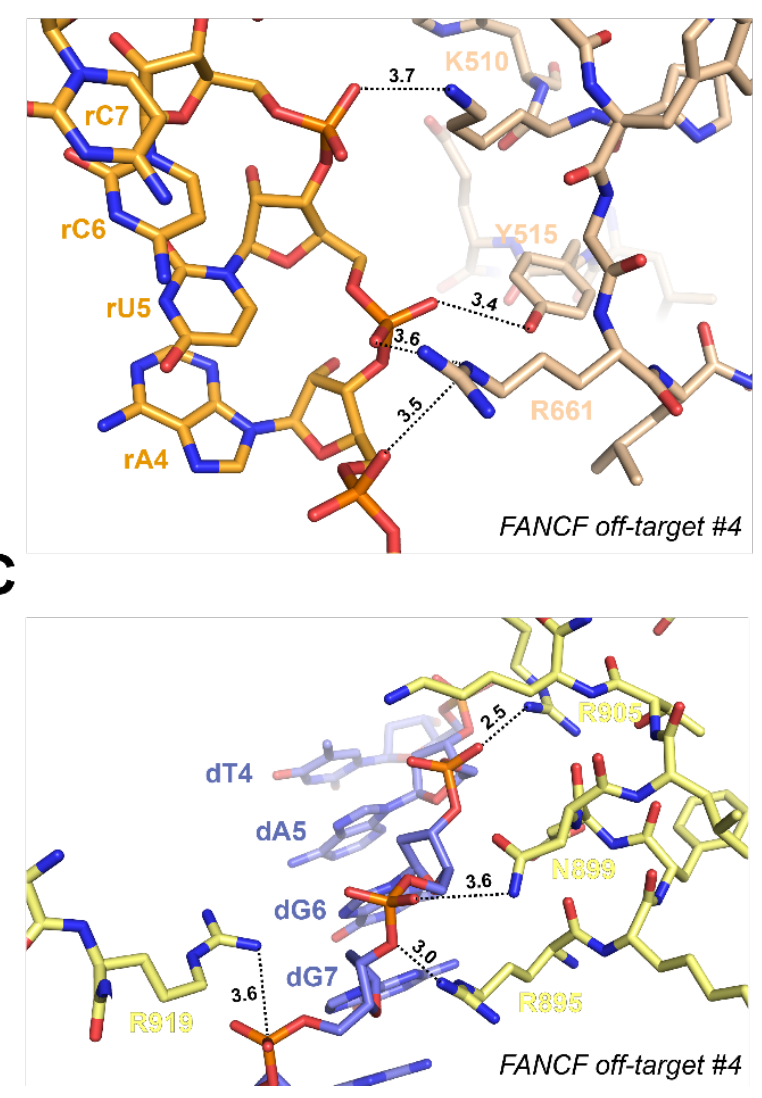

D

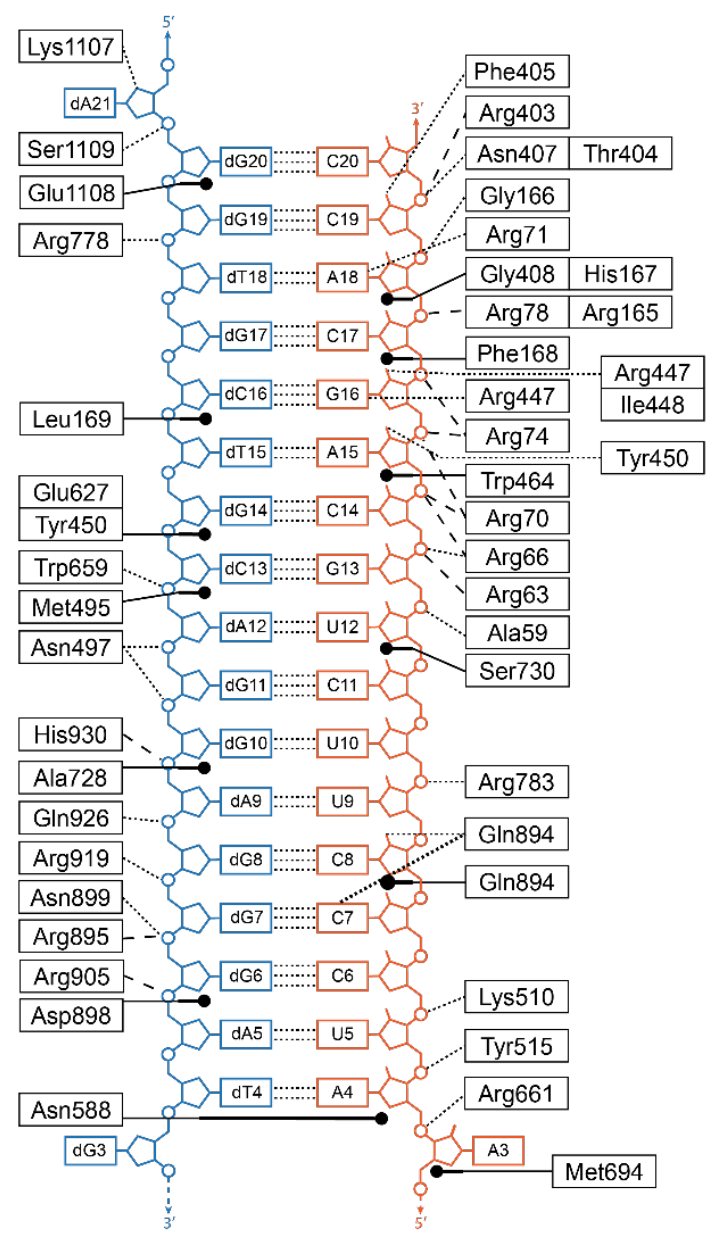

FANCF off-target \#4

Hydrogen bond

- van der Waals interaction

... - . Salt bridge

Figure S16. Altered heteroduplex interactions in FANCF off-target \#4 complex.

908 (A) Overlay of REC3 domain helix 703-712 in FANCF off-target \#4 complex (wheat) with

FANCF on-target complex (orange). (B) Close-up view of REC3 domain interactions with the guide RNA strand in FANCF off-target \#4 complex. (C) Close-up view of TS DNA interactions

911 established by $\mathrm{HNH}$ domain in FANCF off-target \#4 complex. (D) Schematic diagram

912 depicting Cas9 residues interacting with the guide RNA-off-target DNA heteroduplex in

913 FANCF off-target \#4 complex. Dotted lines represent hydrogen bonding interactions, dashed

914 lines represent salt bridges, solid lines represent stacking/hydrophobic interactions. Target 
915 strand is coloured blue, guide RNA orange. Phosphates are represented by circles, ribose

916 moieties by pentagons, and nucleobases by rectangles.

917 


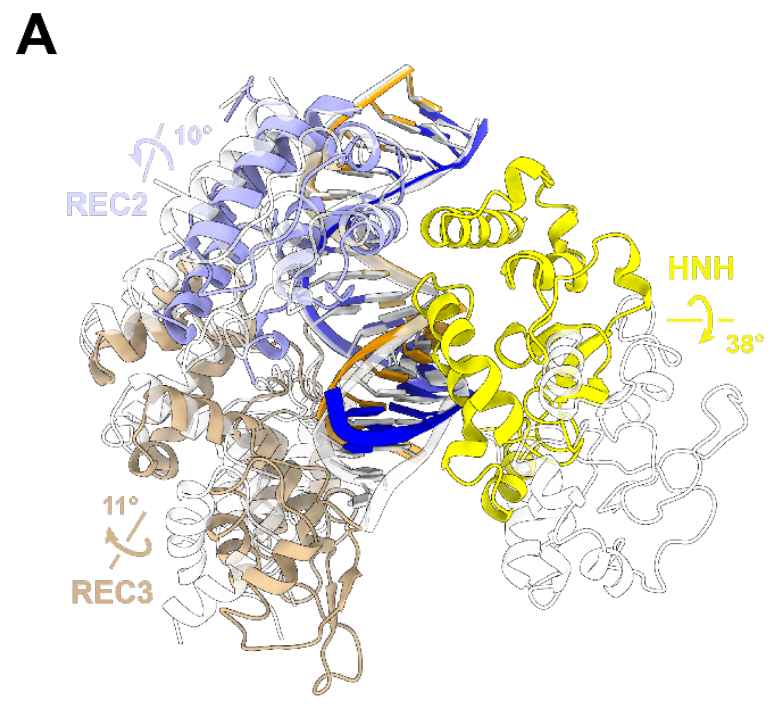

FANCF off-target4 vs.

pre-catalytic complex (PDB: 6O0Z)
B
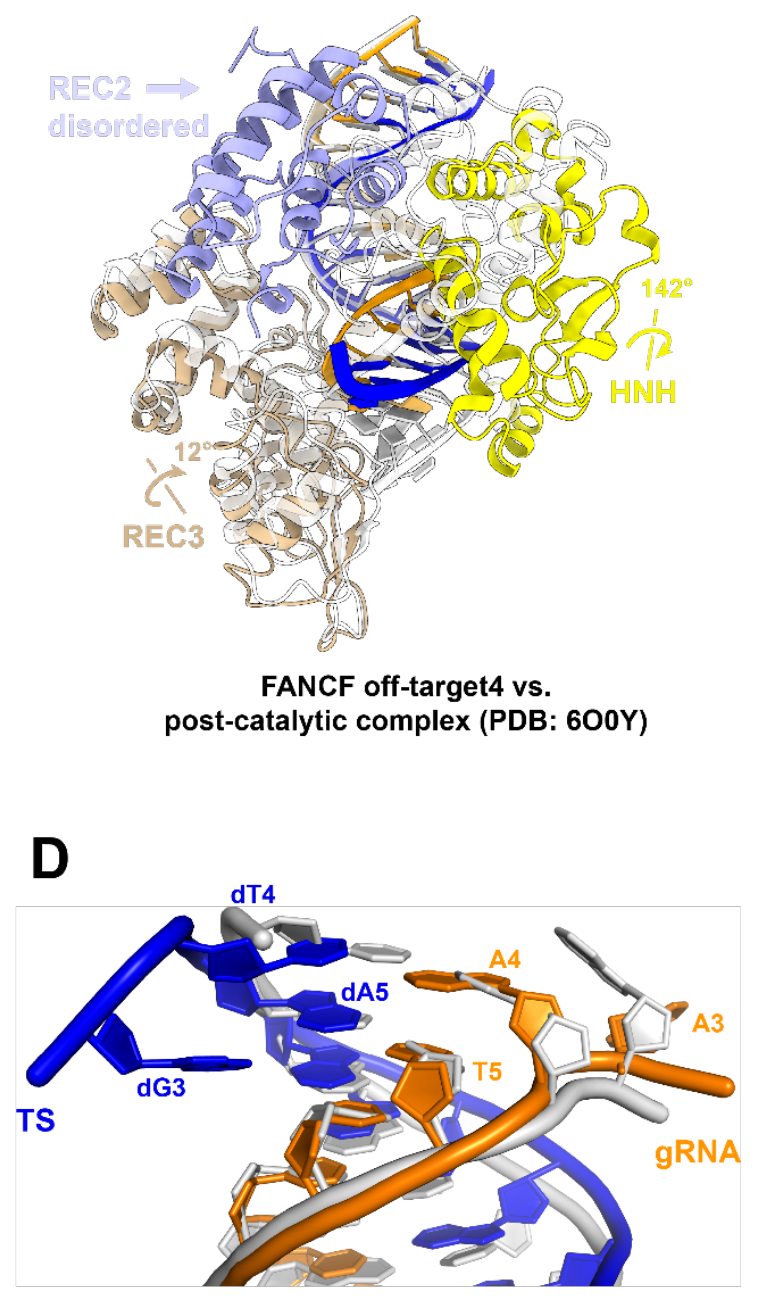

FANCF off-target4
C

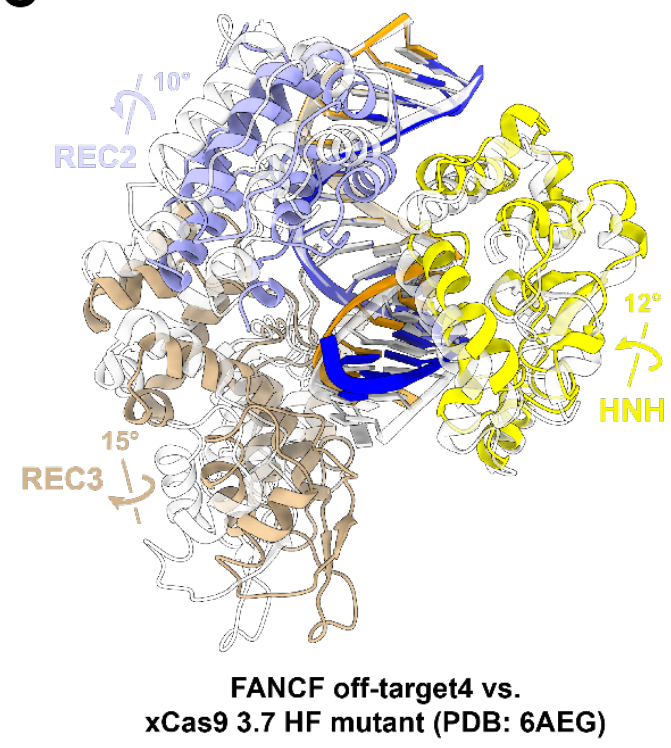

\section{Figure S17}

\section{Figure S17. Conformational rearrangements of REC2/3 AND HNH domains in FANCF}

off-target \#4 complex.

921 (A) Structural overlay of the $F A N C F$ off-target \#4 complex with cryo-EM structure of a pre-

catalytic (State I) Cas9 complex (PDB: 6O0Z). (B) Structural overlay of the FANCF off-target

923 \#4 complex with the cryo-EM structure of a post-catalytic (State II) Cas9 complex (PDB:

924 6O0Y). (C) Structural overlay of the FANCF off-target \#4 complex with the crystallographic 925 structure of the high-fidelity xCas9 3.7 variant (PDB: 6AEG). The REC1, RuvC, and PAM 
926 interaction domains have been omitted for clarity in all panels, as no significant structural 927 changes were observed in these domains. The FANCF off-target \#4 complex domains are 928 colored according to Figure 1A. The overlaid structures are coloured white. (D) Overlay of the 929 PAM-distal heteroduplex region in FANCF off-target \#4 and xCas9 3.7 on-target complexes.

930 Target strand is coloured in blue, guide RNA is coloured orange.

931 
Table S1. SITE-Seq assay results for Cas9 off-target profiling of 12 selected genomic sites.

933 Columns indicate the recovered off-target sequence; motif location; number of substitutions in

934 recovered target sequence compared to the on-target (substitutions); strand designation of

935 PAM; the lowest recovery concentration of each target; and whether the off-target is predicted

936 to contain inserts or deletions based on restricted pipeline paraments. Off-target sites recovered

937 at lower concentrations were also recovered at higher concentrations (e.g., all 4nM sites were

938 also recovered at $16 \mathrm{nM}, 64 \mathrm{nM}$, and $256 \mathrm{nM})$.

939

Table S2. List of recovered off-target sequences aligned to the corresponding on-target

941 sequence.

942 Off-target alignments classified by genomic target and by the presence of insertions, deletions

943 or purely mismatched targets, as based on restricted pipeline paraments. Indexes correspond to 944 off-target sequence numbering in Table S1.

Table S3. Crystallographic data collection and refinement statistics of Cas9 on-target and

Table S4. 3DNA 2.0 analysis of the helical parameters and sugar puckering of 
956

957

958

959

960

961

962

963

964

965

966

967

968

969

970

971

972

973

974

975

976

977

978

979

980

\section{Methods}

\section{DNA oligonucleotides and substrates}

Sequences of DNA oligonucleotides used in this study are summarised in Table S5. Crystallisation substrates were synthesised by Sigma Aldrich without further purification, sgRNA transcription templates and ATTO-532 labelled cleavage substrates were synthesised by Integrated DNA Technologies, Inc., with PAGE and HPLC purification, respectively. Partially double stranded crystallisation substrates were prepared by mixing complementary oligonucleotides in a $1: 1$ molar ratio (as determined by $260 \mathrm{~nm}$ absorption), heating to $95{ }^{\circ} \mathrm{C}$ for 5 minutes and slow cooling to room-temperature. Cleavage substrates were prepared similarly, except that a 2-fold molar excess of the non-target strand was used.

\section{Cas9 protein expression and purification}

Streptococcus pyogenes Cas9 wild type protein and the nuclease dead mutant (D10A, H840A) were both recombinantly expressed for 16 hours at $18{ }^{\circ} \mathrm{C}$ in Escherichia coli Rosetta 2 (DE3) (Novagen) N-terminally fused to a hexahistidine affinity tag, the maltose binding protein (MBP) polypeptide, and the tobacco etch virus (TEV) protease cleavage site. Cells were resuspended and lysed in $20 \mathrm{mM}$ HEPES-KOH pH 7.5, $500 \mathrm{mM} \mathrm{KCl,} 5 \mathrm{mM}$ imidazole, and supplemented with added protease inhibitors. Clarified lysate was loaded on a $10 \mathrm{ml}$ Ni-NTA Superflow column (QIAGEN), washed with 7 column volumes of $20 \mathrm{mM}$ HEPES-KOH $\mathrm{pH}$ 7.5, $500 \mathrm{mM} \mathrm{KCl}, 5 \mathrm{mM}$ imidazole, and eluted with 10 column volumes of $20 \mathrm{mM}$ HEPES$\mathrm{KOH} \mathrm{pH} 7.5,250 \mathrm{mM} \mathrm{KCl}, 200 \mathrm{mM}$ imidazole. Salt concentration is adjusted and protein is loaded on a $10 \mathrm{ml}$ HiTrap Heparin HP column (GE Healthcare) equilibrated in 20 mM HEPES$\mathrm{KOH} \mathrm{pH} 7.5,250 \mathrm{mM} \mathrm{KCl}, 1 \mathrm{mM}$ DTT. The column is washed with 5 column volumes of 20 mM HEPES-KOH pH 7.5, $250 \mathrm{mM} \mathrm{KCl}, 1 \mathrm{mM}$ DTT, and Cas9 is eluted with 17 column volumes of $20 \mathrm{mM}$ HEPES-KOH pH 7.5, $1.5 \mathrm{M} \mathrm{KCl}, 1 \mathrm{mM}$ DTT, in a 0-32\% gradient (peak elution around $500 \mathrm{mM} \mathrm{KCl}$ ). His6-MBP tag was removed by TEV protease cleavage overnight 
981 with gentle shaking. The untagged Cas9 was concentrated and applied to a Superdex 200 16/600

982 (GE Healthcare) and eluted with $20 \mathrm{mM}$ HEPES-KOH pH 7.5, $500 \mathrm{mM} \mathrm{KCl,} 1 \mathrm{mM}$ DTT.

983 Purified protein was concentrated to $10 \mathrm{mg} / \mathrm{ml}$, flash frozen in liquid nitrogen and store

984 at $-80{ }^{\circ} \mathrm{C}$. DTT was omitted in the size-exclusion step of the purification when protein was used

985 for switchSENSE measurements.

986 sgRNA transcription and purification

987 sgRNAs are transcribed from a double stranded PCR product template amplified from a plasmid 988 in a $5 \mathrm{ml}$ transcription reaction $(30 \mathrm{mM}$ Tris- $\mathrm{HCl} \mathrm{pH} 8.1,25 \mathrm{mM} \mathrm{MgCl}, 2 \mathrm{mM}$ spermidine, $9890.01 \%$ Triton X-100, 5 mM CTP, 5 mM ATP, 5 mM GTP, 5 mM UTP, 10 mM DTT, $1 \mu \mathrm{M}$ 990 DNA transcription template, 0.5 units inorganic pyrophosphatase (Thermo Fischer), $250 \mu \mathrm{g}$ 991 homemade T7 RNA polymerase. The reaction is incubated at $37^{\circ} \mathrm{C}$ for 5 hours, and then treated 992 for 30 minutes with 15 units of RQ1 DNAse (Promega). The transcribed sgRNAs are 993 subsequently PAGE purified on an 8\% denaturing (7 M urea) polyacrylamide gel, and lastly 994 ethanol precipitated and resuspended in DEPC treated water.

To assemble the Cas9 on-/off-target ternary complexes, the Cas9 protein is first mixed with the 997 sgRNA in a 1:1.5 molar ratio and incubated at room temperature for 10 minutes. Next, the 998 binary complex is diluted to $2 \mathrm{mg} / \mathrm{ml}$ with $20 \mathrm{mM}$ HEPES-KOH 7.5, $250 \mathrm{mM} \mathrm{KCl}, 1 \mathrm{mM} \mathrm{DTT}$, $2 \mathrm{mM} \mathrm{MgCl} 2$ buffer, pre-annealed $100 \mu \mathrm{M}$ DNA substrate is added in a 1:1.8 molar ratio and

1000 the complex is incubated another 10 minutes at room temperature. For crystallisation, $1 \mu 1$ of 1001 the ternary complex $(1-2 \mathrm{mg} / \mathrm{ml})$ is mixed with $1 \mu \mathrm{l}$ of the reservoir solution $(0.1 \mathrm{M}$ Tris-acetate $1002 \mathrm{pH} 8.5,0.3-0.5 \mathrm{M} \mathrm{KSCN}, 17-19 \%$ PEG3350) and crystals are grown at $20{ }^{\circ} \mathrm{C}$ using the hanging 1003 drop vapour diffusion method. In some cases, microseeding was be used to improve crystal 1004 morphology. Crystals are typically harvested after 2-3 weeks, cryoprotected in $0.1 \mathrm{M}$ Tris1005 acetate $\mathrm{pH}$ 8.5, 0.4 M KSCN, 30\% PEG3350, 15\% ethylene glycol, $1 \mathrm{mM} \mathrm{MgCl}$, and flash- 
cooled in liquid nitrogen. Diffraction data was obtained at beamlines PXI and PXIII of the

1007 Swiss Light Source (Paul Scherrer Institute, Villigen, Switzerland) and were processed using 1008 the XDS package (Kabsch, 2010). Structures were solved by molecular replacement through 1009 the Phaser module of the Phenix package (Adams et al., 2010) using the PDB ID: 5FQ5 model

1010 omitting the RNA-DNA target duplex from the search. Model adjustment and duplex building 1011 was completed using COOT software (Emsley et al., 2010). Atomic model refinement was 1012 performed using Phenix.refine (Adams et al., 2010). Protein-nucleic acid interactions were 1013 analysed using the PISA web server (Krissinel and Henrick, 2007). Characterisation of the 1014 guide-protospacer duplex was performed using the 3DNA 2.0 web server (Li et al., 2019). 1015 Structural figures were generated using PyMOL and ChimeraX (Pettersen et al., 2021).

1016 In vitro nuclease activity assays

1017 Cleavage reactions were performed at $37{ }^{\circ} \mathrm{C}$ in reaction buffer, containing $20 \mathrm{mM} \mathrm{HEPES} \mathrm{pH}$ $10187.5,250 \mathrm{mM} \mathrm{KCl}, 5 \mathrm{mM} \mathrm{MgCl} 2$ and $1 \mathrm{mM}$ DTT. First, Cas9 protein was pre-incubated with 1019 sgRNA in 1:1.25 ratio for 10 minutes at room temperature. The protein-RNA complex was 1020 rapidly mixed with the ATTO-532 labelled dsDNA, to yield final concentrations of $1.67 \mu \mathrm{M}$ 1021 protein and $66.67 \mathrm{nM}$ substrate in a $7.5 \mu 1$ reaction. Time points were harvested at $1,2.5,5,15$, $102245,90,150$ minutes, and 24 hours. Cleavage was stopped by addition of $2 \mu 1$ of $250 \mathrm{mM}$ EDTA, $10230.5 \%$ SDS and $20 \mu \mathrm{g}$ of Proteinase K. Formamide was added to the reactions with final 1024 concentration of $50 \%$, samples were incubated at $95{ }^{\circ} \mathrm{C}$ for 10 minutes, and resolved on a $15 \%$ 1025 denaturing PAGE gel containing 7M urea and imaged using a Typhoon FLA 9500 gel imager. 1026 Depicted error bars correspond to the standard deviation from four independent cleavage 1027 reactions. Rate constants $\left(\mathrm{k}_{\mathrm{obs}}\right)$ were extracted from single exponential fits: [Product] $=\mathrm{A}^{*}(1$ $\left.1028 \exp \left(-\mathrm{k}_{\mathrm{obs}} * \mathrm{t}\right)\right)$ 
1030 The target strands (TS) containing a 3' flanking sequence complementary to the ssDNA

1031 covalently bound to the chip electrode, and the non-target strands (NTS) (Table S5) were

1032 resuspended in a buffer containing $10 \mathrm{mM}$ Tris- $\mathrm{HCl} \mathrm{pH} 7.4,40 \mathrm{mM} \mathrm{NaCl}$, and $0.05 \%$ Tween

1033 20. The matching TS:NTS duplex is pre-annealed and hybridised to the chip anode. The Cas9

1034 protein was mixed with the sgRNAs at a 1:2 protein:RNA molar ratio, and the complex was

1035 incubated for $30 \mathrm{~min}$ at $37^{\circ} \mathrm{C}$ in association buffer containing $20 \mathrm{mM}$ HEPES-KOH pH 7.5,

$1036150 \mathrm{mM} \mathrm{KCl}, 2 \mathrm{mM} \mathrm{MgCl} 2,0.01 \%$ Tween 20. All switchSENSE experiments were performed

1037 on a DRX analyser using CAS-48-1-R1-S chips (Dynamic Biosensors GmbH, Martinsried,

1038 Germany). Kinetics experiments were performed at $25{ }^{\circ} \mathrm{C}$ in association buffer, with an

1039 association time of $5 \mathrm{~min}$, dissociation time of $20 \mathrm{~min}$, and a flow rate of $50 \mu \mathrm{l} / \mathrm{min}$.

1040 SITE-Seq assay

1041 SITE-Seq assay reaction conditions were performed as described previously (Cameron et al.,

1042 2017). Briefly, high molecular weight genomic DNA (gDNA) was purified from human

1043 primary T cells using the Blood \& Cell Culture DNA Maxi Kit (Qiagen) according to the

1044 manufacturer's instructions. RNPs comprising the guides were biochemically assembled for

1045 gDNA digestion. Specifically, equal molar amounts of crRNA and tracrRNA were mixed and

1046 heated to $95{ }^{\circ} \mathrm{C}$ for $2 \mathrm{~min}$ then allowed to cool at room temperature for $\sim 5 \mathrm{~min}$. Three-fold

1047 molar excess of the guides were incubated with Streptococcus pyogenes Cas9 (SpCas9) in

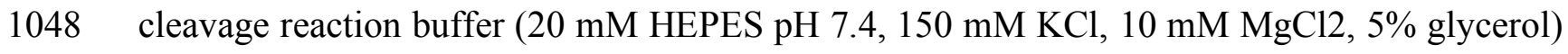

1049 at $37^{\circ} \mathrm{C}$ for $10 \mathrm{~min}$. In a 96-well plate format, $10 \mu \mathrm{g}$ of gDNA was treated with 0.2 pmol (4

$1050 \mathrm{nM}), 0.8 \mathrm{pmol}(16 \mathrm{nM}), 3.2 \mathrm{pmol}(64 \mathrm{nM})$, and $12.8 \mathrm{pmol}(256 \mathrm{nM})$ of each RNP in $50 \mu \mathrm{L}$ total

1051 volume in cleavage reaction buffer. Each cleavage reaction was performed in triplicate.

1052 Negative control reactions were assembled in parallel and did not include RNP. gDNA was

1053 treated with RNPs for 4 hours at $37^{\circ} \mathrm{C}$. SITE-Seq assay library preparation and sequencing 
1054 was performed as described previously and the final library was loaded onto the Illumina

1055 NextSeq platform (Illumina, San Diego, CA), and 1-3 M reads were obtained for each sample.

1057 SITE-Seq assay recovered off-targets were filtered for sites that had read-pileups proximal to

1058 the expected cut site, a PAM comprising at least one guanine base, fewer than 12 mismatches

1059 (reasoning that sites with 12 or more mismatches are likely spurious peaks not resulting from

1060 Cas9-induced double-strand breaks), and all sites with 11 mismatches were visually inspected

1061 and included in analysis if a putative deletion or insertion would result in a reduction of $>4$

1062 mismatches relative to the spacer sequence.

1063 In silico mismatch, deletion, and insertion prediction algorithm

1064 Predictive classification of SITE-Seq assay recovered off-target sites as pure mismatches,

1065 deletions, or insertions was executed using a scoring algorithm which consisted of the following 1066 sequential steps (Figure S14):

1067 (i) For each off-target, a gap library was generated where a single nucleotide gap was introduced between each nucleotide in the off-target sequence. improved alignment by at least 4 nucleotides relative to the non-gapped spacer-offtarget alignment, the off-target sequence was marked as a single-nucleotide deletion and removed from subsequent analysis.

(iii) The remaining pool of off-targets were then aligned to a spacer gapped library where a single nucleotide gap was introduced at each positing in the spacer. 
and the gapped spacer pair. If the gapped spacer with the highest alignment score improved alignment by at least 4 nucleotides relative to the non-gapped spacer-offtarget alignment, the off-target sequence was marked as a single-nucleotide insertion and removed from subsequent analysis.

(v) The remaining off-target for which the spacer-off-target alignment was not improved by single-nucleotide deletions or insertions were annotated as a mismatched off-target.

The prediction pipeline process was the same for the 'unrestricted' and structurally-

1087 informed 'restricted' pipelines, however in the 'restricted' pipeline the deletion gap library 1088 was restricted to positions 10-20 and the insertion gap library was restricted to positions 620. 


\section{References}

1093

1094

1095

1096

1097

1098

1099

1100

1101

1102

1103

1104

1105

1106

1107

1108

1109

1110

1111

1112

1113

1114

1115

1116

1117

1118

1119

1120

1121

1122

1123

1124

1125

1126

1127

1128

1129

1130

1131

1132

1133

Abe, N., Dror, I., Yang, L., Slattery, M., Zhou, T., Bussemaker, H.J., Rohs, R., and Mann, R.S. (2015). Deconvolving the recognition of DNA shape from sequence. Cell 161, 307-318.

Adams, P.D., Afonine, P.V., Bunkoczi, G., Chen, V.B., Davis, I.W., Echols, N., Headd, J.J., Hung, L.W., Kapral, G.J., Grosse-Kunstleve, R.W., et al. (2010). PHENIX: a comprehensive Python-based system for macromolecular structure solution. Acta Crystallogr D Biol Crystallogr 66, 213-221.

Afek, A., Shi, H., Rangadurai, A., Sahay, H., Senitzki, A., Xhani, S., Fang, M., Salinas, R., Mielko, Z., Pufall, M.A., et al. (2020). DNA mismatches reveal conformational penalties in protein-DNA recognition. Nature.

Anders, C., Niewoehner, O., Duerst, A., and Jinek, M. (2014). Structural basis of PAM-dependent target DNA recognition by the Cas9 endonuclease. Nature 513, 569-573.

Anzalone, A.V., Koblan, L.W., and Liu, D.R. (2020). Genome editing with CRISPR-Cas nucleases, base editors, transposases and prime editors. Nat Biotechnol 38, 824-844.

Bae, S., Park, J., and Kim, J.S. (2014). Cas-OFFinder: a fast and versatile algorithm that searches for potential off-target sites of Cas9 RNA-guided endonucleases. Bioinformatics 30, 1473-1475.

Boyle, E.A., Andreasson, J.O.L., Chircus, L.M., Sternberg, S.H., Wu, M.J., Guegler, C.K., Doudna, J.A., and Greenleaf, W.J. (2017). High-throughput biochemical profiling reveals sequence determinants of dCas9 off-target binding and unbinding. Proc Natl Acad Sci U S A 114, 5461-5466.

Boyle, E.A., Becker, W.R., Bai, H.B., Chen, J.S., Doudna, J.A., and Greenleaf, W.J. (2021). Quantification of Cas9 binding and cleavage across diverse guide sequences maps landscapes of target engagement. Science Advances 7, eabe5496.

Bravo, J.P.K., Liu, M.-S., McCool, R.S., Jung, K., Johnson, K.A., and Taylor, D.W. (2021). Structural basis for mismatch surveillance by CRISPR/Cas9. bioRxiv, 2021.2009.2014.460224.

Cameron, P., Fuller, C.K., Donohoue, P.D., Jones, B.N., Thompson, M.S., Carter, M.M., Gradia, S., Vidal, B., Garner, E., Slorach, E.M., et al. (2017). Mapping the genomic landscape of CRISPR-Cas9 cleavage. Nat Methods 14, 600-606.

Chen, J.S., Dagdas, Y.S., Kleinstiver, B.P., Welch, M.M., Sousa, A.A., Harrington, L.B., Sternberg, S.H., Joung, J.K., Yildiz, A., and Doudna, J.A. (2017). Enhanced proofreading governs CRISPR-Cas9 targeting accuracy. Nature 550, 407-410.

Cofsky, J.C., Soczek, K.M., Knott, G.J., Nogales, E., and Doudna, J.A. (2021). CRISPR-Cas9 bends and twists DNA to read its sequence. bioRxiv, 2021.2009.2006.459219.

Dagdas, Y.S., Chen, J.S., Sternberg, S.H., Doudna, J.A., and Yildiz, A. (2017). A conformational checkpoint between DNA binding and cleavage by CRISPR-Cas9. Sci Adv 3, eaao0027.

Deltcheva, E., Chylinski, K., Sharma, C.M., Gonzales, K., Chao, Y., Pirzada, Z.A., Eckert, M.R., Vogel, J., and Charpentier, E. (2011). CRISPR RNA maturation by trans-encoded small RNA and host factor RNase III. Nature 471, 602-607.

Deveau, H., Barrangou, R., Garneau, J.E., Labonte, J., Fremaux, C., Boyaval, P., Romero, D.A., Horvath, P., and Moineau, S. (2008). Phage response to CRISPR-encoded resistance in Streptococcus thermophilus. J Bacteriol 190, 1390-1400.

Doench, J.G., Fusi, N., Sullender, M., Hegde, M., Vaimberg, E.W., Donovan, K.F., Smith, I., Tothova, Z., Wilen, C., Orchard, R., et al. (2016). Optimized sgRNA design to maximize activity and minimize off-target effects of CRISPR-Cas9. Nat Biotechnol 34, 184-191. 
Donohoue, P.D., Pacesa, M., Lau, E., Vidal, B., Irby, M.J., Nyer, D.B., Rotstein, T., Banh, L., Toh, M.S., Gibson, J., et al. (2021). Conformational control of Cas9 by CRISPR hybrid RNA-DNA guides mitigates off-target activity in T cells. Mol Cell 81, 3637-3649 e3635.

Emsley, P., Lohkamp, B., Scott, W.G., and Cowtan, K. (2010). Features and development of Coot. Acta Crystallogr D Biol Crystallogr 66, 486-501.

1139 Fu, B.X.H., Smith, J.D., Fuchs, R.T., Mabuchi, M., Curcuru, J., Robb, G.B., and Fire, A.Z. (2019). 897.

Fu, Y., Sander, J.D., Reyon, D., Cascio, V.M., and Joung, J.K. (2014). Improving CRISPR-Cas nuclease specificity using truncated guide RNAs. Nat Biotechnol 32, 279-284.

Garg, A., and Heinemann, U. (2018). A novel form of RNA double helix based on G.U and C.A(+) wobble base pairing. RNA 24, 209-218.

1146 Gong, S., Yu, H.H., Johnson, K.A., and Taylor, D.W. (2018). DNA Unwinding Is the Primary Determinant of CRISPR-Cas9 Activity. Cell Rep 22, 359-371.

1148 Guo, M., Ren, K., Zhu, Y., Tang, Z., Wang, Y., Zhang, B., and Huang, Z. (2019). Structural insights into a high fidelity variant of SpCas9. Cell Res 29, 183-192.

1150 Hsu, P.D., Scott, D.A., Weinstein, J.A., Ran, F.A., Konermann, S., Agarwala, V., Li, Y., Fine, E.J., Wu, 1151 X., Shalem, O., et al. (2013). DNA targeting specificity of RNA-guided Cas9 nucleases. Nat Biotechnol $1152 \quad 31,827-832$.

Ivanov, I.E., Wright, A.V., Cofsky, J.C., Aris, K.D.P., Doudna, J.A., and Bryant, Z. (2020). Cas9 interrogates DNA in discrete steps modulated by mismatches and supercoiling. Proc Natl Acad Sci U S A $117,5853-5860$.

Jiang, F., Taylor, D.W., Chen, J.S., Kornfeld, J.E., Zhou, K., Thompson, A.J., Nogales, E., and Doudna, J.A. (2016). Structures of a CRISPR-Cas9 R-loop complex primed for DNA cleavage. Science 351, 867-871.

1159 Jiang, F., Zhou, K., Ma, L., Gressel, S., and Doudna, J.A. (2015). STRUCTURAL BIOLOGY. A Cas9guide RNA complex preorganized for target DNA recognition. Science 348, 1477-1481.

1161

Jinek, M., Chylinski, K., Fonfara, I., Hauer, M., Doudna, J.A., and Charpentier, E. (2012). A programmable dual-RNA-guided DNA endonuclease in adaptive bacterial immunity. Science 337, 816821.

1164

Jones, S.K., Jr., Hawkins, J.A., Johnson, N.V., Jung, C., Hu, K., Rybarski, J.R., Chen, J.S., Doudna, J.A., Press, W.H., and Finkelstein, I.J. (2020). Massively parallel kinetic profiling of natural and engineered CRISPR nucleases. Nat Biotechnol.

1167 Kabsch, W. (2010). Xds. Acta Crystallogr D Biol Crystallogr 66, 125-132.

Kimsey, I.J., Petzold, K., Sathyamoorthy, B., Stein, Z.W., and Al-Hashimi, H.M. (2015). Visualizing transient Watson-Crick-like mispairs in DNA and RNA duplexes. Nature 519, 315-320.

Kimsey, I.J., Szymanski, E.S., Zahurancik, W.J., Shakya, A., Xue, Y., Chu, C.C., Sathyamoorthy, B., Suo, Z., and Al-Hashimi, H.M. (2018). Dynamic basis for $\mathrm{dG}^{*} \mathrm{dT}$ misincorporation via tautomerization and ionization. Nature 554, 195-201.

1176 Krissinel, E., and Henrick, K. (2007). Inference of macromolecular assemblies from crystalline state. J 
Kulcsar, P.I., Talas, A., Toth, E., Nyeste, A., Ligeti, Z., Welker, Z., and Welker, E. (2020). Blackjack mutations improve the on-target activities of increased fidelity variants of SpCas9 with 5'G-extended sgRNAs. Nat Commun 11, 1223.

Kunkel, T.A., and Bebenek, K. (2000). DNA replication fidelity. Annu Rev Biochem 69, 497-529.

Kuscu, C., Arslan, S., Singh, R., Thorpe, J., and Adli, M. (2014). Genome-wide analysis reveals characteristics of off-target sites bound by the Cas9 endonuclease. Nat Biotechnol 32, 677-683. Jividen, K., et al. (2020). CHANGE-seq reveals genetic and epigenetic effects on CRISPR-Cas9 genome-wide activity. Nat Biotechnol.

1187 Leontis, N.B., Stombaugh, J., and Westhof, E. (2002). The non-Watson-Crick base pairs and their associated isostericity matrices. Nucleic Acids Res 30, 3497-3531.

Li, S., Olson, W.K., and Lu, X.J. (2019). Web 3DNA 2.0 for the analysis, visualization, and modeling of 3D nucleic acid structures. Nucleic Acids Res 47, W26-W34.

Lin, Y., Cradick, T.J., Brown, M.T., Deshmukh, H., Ranjan, P., Sarode, N., Wile, B.M., Vertino, P.M., Stewart, F.J., and Bao, G. (2014). CRISPR/Cas9 systems have off-target activity with insertions or deletions between target DNA and guide RNA sequences. Nucleic Acids Res 42, 7473-7485.

Makarova, K.S., Wolf, Y.I., Iranzo, J., Shmakov, S.A., Alkhnbashi, O.S., Brouns, S.J.J., Charpentier, E., Cheng, D., Haft, D.H., Horvath, P., et al. (2020). Evolutionary classification of CRISPR-Cas systems: a burst of class 2 and derived variants. Nat Rev Microbiol 18, 67-83.

Mekler, V., Minakhin, L., and Severinov, K. (2017). Mechanism of duplex DNA destabilization by RNA-guided Cas9 nuclease during target interrogation. Proc Natl Acad Sci U S A 114, 5443-5448.

Mitchell, B.P., Hsu, R.V., Medrano, M.A., Zewde, N.T., Narkhede, Y.B., and Palermo, G. (2020). Spontaneous Embedding of DNA Mismatches Within the RNA:DNA Hybrid of CRISPR-Cas9. Frontiers in Molecular Biosciences 7.

1202 Mullally, G., van Aelst, K., Naqvi, M.M., Diffin, F.M., Karvelis, T., Gasiunas, G., Siksnys, V., and Szczelkun, M.D. (2020). 5' modifications to CRISPR-Cas9 gRNA can change the dynamics and size of R-loops and inhibit DNA cleavage. Nucleic Acids Res.

Murugan, K., Seetharam, A.S., Severin, A.J., and Sashital, D.G. (2020). High-throughput <em>in vitro $</$ em $>$ specificity profiling of natural and high-fidelity CRISPR-Cas9 variants. bioRxiv, 2020.2005.2012.091991.

1211 O'Connell, M.R., Oakes, B.L., Sternberg, S.H., East-Seletsky, A., Kaplan, M., and Doudna, J.A. (2014). 1212 Programmable RNA recognition and cleavage by CRISPR/Cas9. Nature 516, 263-266.

1213 O'Geen, H., Henry, I.M., Bhakta, M.S., Meckler, J.F., and Segal, D.J. (2015). A genome-wide analysis 1214 of Cas9 binding specificity using ChIP-seq and targeted sequence capture. Nucleic Acids Res 43, 338912153404. (2019). Single molecule analysis of effects of non-canonical guide RNAs and specificity-enhancing

1219 Pacesa, M., and Jinek, M. (2021). Mechanism of R-loop formation and conformational activation of 1220 Cas9. bioRxiv, 2021.2009.2016.460614. 
Palermo, G., Chen, J.S., Ricci, C.G., Rivalta, I., Jinek, M., Batista, V.S., Doudna, J.A., and McCammon, J.A. (2018). Key role of the REC lobe during CRISPR-Cas9 activation by 'sensing', 'regulating', and 'locking' the catalytic HNH domain. Q Rev Biophys 51.

Pattanayak, V., Lin, S., Guilinger, J.P., Ma, E., Doudna, J.A., and Liu, D.R. (2013). High-throughput profiling of off-target DNA cleavage reveals RNA-programmed Cas9 nuclease specificity. Nat Biotechnol 31, 839-843.

Pettersen, E.F., Goddard, T.D., Huang, C.C., Meng, E.C., Couch, G.S., Croll, T.I., Morris, J.H., and Ferrin, T.E. (2021). UCSF ChimeraX: Structure visualization for researchers, educators, and developers. Protein Sci 30, 70-82.

Ricci, C.G., Chen, J.S., Miao, Y., Jinek, M., Doudna, J.A., McCammon, J.A., and Palermo, G. (2019). Deciphering Off-Target Effects in CRISPR-Cas9 through Accelerated Molecular Dynamics. ACS Cent Sci 5, 651-662.

Rodnina, M.V., and Wintermeyer, W. (2001). Fidelity of aminoacyl-tRNA selection on the ribosome: kinetic and structural mechanisms. Annu Rev Biochem 70, 415-435.

Rohs, R., West, S.M., Liu, P., and Honig, B. (2009a). Nuance in the double-helix and its role in proteinDNA recognition. Curr Opin Struct Biol 19, 171-177.

Rohs, R., West, S.M., Sosinsky, A., Liu, P., Mann, R.S., and Honig, B. (2009b). The role of DNA shape in protein-DNA recognition. Nature 461, 1248-1253.

Semenova, E., Jore, M.M., Datsenko, K.A., Semenova, A., Westra, E.R., Wanner, B., van der Oost, J., Brouns, S.J., and Severinov, K. (2011). Interference by clustered regularly interspaced short palindromic repeat (CRISPR) RNA is governed by a seed sequence. Proc Natl Acad Sci U S A 108, 10098-10103.

Singh, D., Sternberg, S.H., Fei, J., Doudna, J.A., and Ha, T. (2016). Real-time observation of DNA recognition and rejection by the RNA-guided endonuclease Cas9. Nat Commun 7, 12778.

Stemmer, M., Thumberger, T., Del Sol Keyer, M., Wittbrodt, J., and Mateo, J.L. (2015). CCTop: An Intuitive, Flexible and Reliable CRISPR/Cas9 Target Prediction Tool. PLoS One 10, e0124633.

Sternberg, S.H., LaFrance, B., Kaplan, M., and Doudna, J.A. (2015). Conformational control of DNA target cleavage by CRISPR-Cas9. Nature 527, 110-113.

Sternberg, S.H., Redding, S., Jinek, M., Greene, E.C., and Doudna, J.A. (2014). DNA interrogation by the CRISPR RNA-guided endonuclease Cas9. Nature 507, 62-67.

Timsit, Y. (1999). DNA structure and polymerase fidelity. J Mol Biol 293, 835-853.

Tsai, S.Q., Nguyen, N.T., Malagon-Lopez, J., Topkar, V.V., Aryee, M.J., and Joung, J.K. (2017). CIRCLE-seq: a highly sensitive in vitro screen for genome-wide CRISPR-Cas9 nuclease off-targets. Nat Methods 14, 607-614.

Tsai, S.Q., Zheng, Z., Nguyen, N.T., Liebers, M., Topkar, V.V., Thapar, V., Wyvekens, N., Khayter, C., Iafrate, A.J., Le, L.P., et al. (2015). GUIDE-seq enables genome-wide profiling of off-target cleavage by CRISPR-Cas nucleases. Nat Biotechnol 33, 187-197.

van Houte, S., Ekroth, A.K., Broniewski, J.M., Chabas, H., Ashby, B., Bondy-Denomy, J., Gandon, S., Boots, M., Paterson, S., Buckling, A., et al. (2016). The diversity-generating benefits of a prokaryotic adaptive immune system. Nature 532, 385-388.

Vlot, M., Houkes, J., Lochs, S.J.A., Swarts, D.C., Zheng, P., Kunne, T., Mohanraju, P., Anders, C., Jinek, M., van der Oost, J., et al. (2018). Bacteriophage DNA glucosylation impairs target DNA binding by type I and II but not by type V CRISPR-Cas effector complexes. Nucleic Acids Res 46, 873-885. Wang, W., Hellinga, H.W., and Beese, L.S. (2011). Structural evidence for the rare tautomer hypothesis of spontaneous mutagenesis. Proc Natl Acad Sci U S A 108, 17644-17648.

Wu, X., Scott, D.A., Kriz, A.J., Chiu, A.C., Hsu, P.D., Dadon, D.B., Cheng, A.W., Trevino, A.E., Konermann, S., Chen, S., et al. (2014). Genome-wide binding of the CRISPR endonuclease Cas9 in mammalian cells. Nat Biotechnol 32, 670-676. 
1268 Yakovchuk, P., Protozanova, E., and Frank-Kamenetskii, M.D. (2006). Base-stacking and base-pairing contributions into thermal stability of the DNA double helix. Nucleic Acids Res 34, 564-574. Yang, M., Peng, S., Sun, R., Lin, J., Wang, N., and Chen, C. (2018). The Conformational Dynamics of Cas9 Governing DNA Cleavage Are Revealed by Single-Molecule FRET. Cell Rep 22, 372-382. Yaung, S.J., Esvelt, K.M., and Church, G.M. (2014). CRISPR/Cas9-mediated phage resistance is not impeded by the DNA modifications of phage T4. PLoS One 9, e98811.

1274 Zeng, Y., Cui, Y., Zhang, Y., Zhang, Y., Liang, M., Chen, H., Lan, J., Song, G., and Lou, J. (2018). The initiation, propagation and dynamics of CRISPR-SpyCas9 R-loop complex. Nucleic Acids Res 46, 350-

1276361.

1277 Zhang, L., Rube, H.T., Vakulskas, C.A., Behlke, M.A., Bussemaker, H.J., and Pufall, M.A. (2020).

1278 Systematic in vitro profiling of off-target affinity, cleavage and efficiency for CRISPR enzymes. Nucleic 1279 Acids Research.

1280 Zhu, X., Clarke, R., Puppala, A.K., Chittori, S., Merk, A., Merrill, B.J., Simonovic, M., and Subramaniam, S. (2019). Cryo-EM structures reveal coordinated domain motions that govern DNA cleavage by Cas9. Nat Struct Mol Biol 26, 679-685. 